\title{
THE STOCHASTIC THIN-FILM EQUATION: EXISTENCE OF NONNEGATIVE MARTINGALE SOLUTIONS
}

\author{
BENJAMIN GESS AND MANUEL V. GNANN
}

\begin{abstract}
We consider the stochastic thin-film equation with colored Gaussian Stratonovich noise in one space dimension and establish the existence of nonnegative weak (martingale) solutions. The construction is based on a Trotter-Kato-type decomposition into a deterministic and a stochastic evolution, which yields an easy to implement numerical algorithm. Compared to previous work, no interface potential has to be included, the initial data and the solution can have de-wetted regions of positive measure, and the Trotter-Kato scheme allows for a simpler proof of existence than in case of Itô noise.
\end{abstract}

\section{Contents}

1. Introduction

1.1. Setting

1.2. Itô versus Stratonovich formulation 2

1.3. Weak formulation and main result 3

1.4. Decomposition of the dynamics 5

1.5. Outline 6

1.6. Notation and conventions 6

2. Deterministic dynamics 8

3. Stochastic dynamics 9

4. Regularity in time and uniform bounds of approximate solutions 12

5. Convergence of the splitting scheme 16

5.1. Tightness and convergence of a subsequence 16

5.2. Recovering the SPDE 23

6. Concluding remarks 28

Appendix A. Viscous regularization of stochastic dynamics 29

Appendix B. Real interpolation of Besov spaces with mixed smoothness 35

References $\quad 36$

\section{INTRODUCTION}

1.1. Setting. Consider the stochastic thin-film equation with quadratic mobility

$$
\mathrm{d} u=-\partial_{x}\left(u^{2} \partial_{x}^{3} u\right) \mathrm{d} t+\partial_{x}(u \circ \mathrm{d} W) \quad \text { for } \quad(t, x) \in[0, T) \times \mathbb{T}_{L},
$$

Date: July 21, 2020.

BG appreciates discussions with Günther Grün. MVG acknowledges discussions with Christian Kuehn, is obliged to Jonas Sauer for answering a question regarding interpolation of operators, and thanks Heidelberg University, the Max Planck Institute for Mathematics in the Sciences, and the Technical University of Munich for their kind hospitality. The authors thank Konstantinos Dareiotis, Alexandra Neamtu, and the anonymous reviewer for critical readings of the manuscript. BG acknowledges support by the Max Planck Society through the Max Planck Research Group Stochastic partial differential equations and by the Deutsche Forschungsgemeinschaft (DFG, German Research Foundation) through the CRC 1283 Taming uncertainty and profiting from randomness and low regularity in analysis, stochastics and their applications. MVG's work was partially funded by the DFG under project \# 334362478. 
where $T, L \in(0, \infty)$ and $\mathbb{T}_{L}$ denotes the torus of the interval $[0, L]$. We will always assume periodic boundary conditions

$$
\partial_{x}^{j} u(\cdot, 0)=\partial_{x}^{j} u(\cdot, L) \quad \text { for } \quad j \in\{0,1,2,3\}
$$

without further mentioning it. Moreover, suppose that periodic nonnegative initial data $u_{0}: \mathbb{T}_{L} \rightarrow$ $[0, \infty)$ are given, satisfying certain regularity properties that we will specify below. Equation (1.1) describes the evolution of the height $u$ of a two-dimensional viscous thin film as a function of time $t$ and lateral position $x$ influenced by thermal noise $W$ and assuming Navier slip at the substrate. The noise $W$ is assumed to be colored Gaussian and the symbol $u \circ \mathrm{d} W$ denotes Stratonovich noise. Equation (1.1) can be regarded as an approximate model to the full stochastic thin-film equation

$$
\mathrm{d} u=-\partial_{x}\left(\left(\ell_{\mathrm{s}} u^{2}+u^{3}\right) \partial_{x}^{3} u\right) \mathrm{d} t+\partial_{x}\left(\sqrt{\ell_{\mathrm{s}} u^{2}+u^{3}} \circ \mathrm{d} W\right) \quad \text { for } \quad(t, x) \in[0, T) \times \mathbb{T}_{L},
$$

where the constant $\ell_{\mathrm{s}}>0$ denotes the slip length. Hence, (1.1) is an approximation of (1.2) for film heights $u$ that are much smaller than the slip length $\ell_{\mathrm{s}}$.

In this paper we prove the existence of nonnegative martingale solutions to (1.1) (cf. Theorem 1.2 and Remark 1.3 below) for initial data $u_{0} \in H^{1}\left(\mathbb{T}_{L}\right)$ such that $u_{0} \geq 0$. The construction is based on the following Trotter-Kato scheme

$$
\partial_{t} v_{N}=-\partial_{x}\left(v_{N}^{2} \partial_{x}^{3} v_{N}\right) \quad \text { for } \quad(t, x) \in[(j-1) \delta, j \delta) \times \mathbb{T}_{L}
$$

and

$$
\mathrm{d} w_{N}=\partial_{x}\left(w_{N} \circ \mathrm{d} W\right) \quad \text { for } \quad(t, x) \in[(j-1) \delta, j \delta) \times \mathbb{T}_{L},
$$

where $\delta:=\frac{T}{N+1}, j \in\{1, \ldots, N+1\}$, and $N \in \mathbb{N}_{0}$, glueing together according to $v_{N}(0, \cdot):=u_{0}$, $w_{N}((j-1) \delta, \cdot):=\lim _{t \nearrow j \delta} v_{N}(t, \cdot)$ for $j \in\{1, \ldots, N\}$, and $v_{N}(j \delta, \cdot):=\lim _{t \nearrow_{j \delta}} w_{N}(t, \cdot)$ for $j \in\{1, \ldots, N\}$, and taking the limit as $N \rightarrow \infty$. Before giving mathematical details, we will next discuss the choice of Stratonovich instead of Itô noise in (1.1), (1.2), and (1.3b).

1.2. Itô versus Stratonovich formulation. Two versions of the stochastic thin-film equation have been proposed independently. The first due to Davidovitch, Moro, and Stone [17] is in line with the formulation (1.1) and has been applied to describe the enhanced spreading of droplets. The other ground-laying work by Grün, Mecke, and Rauscher [33] additionally takes an interface potential between fluid and substrate into account that prevents $u$ from becoming negative. The study in [33] focuses on coarsening and de-wetting phenomena.

The first rigorous construction of nonnegative martingale solutions to the stochastic thin-film equation with Itô noise and additional interface potential, as derived in [33], has been recently given by Fischer and Grün in [19]. A generalization to more general mobilities at the expense of introducing suitable nonlocal source terms has subsequently been introduced by Cornalba in [13]. The inclusion of an additional interface potential is crucially used in these works in order to obtain suitable a-priori estimates.

The starting point of the (informal) derivation of the stochastic thin-film equation in [33] is the transport equation (see [33, p. 1265, Eq. (6)])

$$
\partial_{t} u=v_{y}-v_{x} \partial_{x} u
$$

where $v_{x}$ and $v_{y}$ denote the horizontal and vertical components of the fluid velocity, respectively. Since the fluid velocity is modelled as a solution to the stochastic incompressible Navier-Stokes equation, it should be understood as a stochastic process. Therefore, the product in (1.4) needs to be understood in the sense of a stochastic integral. We next recall the (informal) derivation of (1.4) in order to justify the choice of stochastic integration (Itô versus Stratonovich). Equation (1.4) can be derived by considering the movement of fluid particles at the liquidair interface with trajectories parametrized by $(x(t), u(t, x(t)))$, where $x(t)$ denotes the lateral 
position as a function of time $t$. The change of the height of the fluid is given by the vertical component $v_{y}$ of the fluid velocity, that is,

$$
\frac{\mathrm{d}}{\mathrm{d} t} u(t, x(t))=v_{y}(x(t), u(t, x(t))) .
$$

The lateral position of a fluid particle changes according to the horizontal component $v_{x}$ of the fluid velocity

$$
\dot{x}(t)=v_{x}(x(t), u(t, x(t))),
$$

which again should be understood as a stochastic equation. Informally, Itô's formula dictates

$$
\begin{aligned}
\frac{\mathrm{d}}{\mathrm{d} t} u(t, x(t)) & =\left(\partial_{t} u\right)(t, x(t))+\left(\partial_{x} u\right)(t, x(t)) \circ \dot{x}(t) \\
& =\left(\partial_{t} u\right)(t, x(t))+\left(\partial_{x} u\right)(t, x(t)) \circ v_{x}(x(t), u(t, x(t))),
\end{aligned}
$$

which together with (1.5) yields (1.4). If we were to use the Itô interpretation in (1.6), an appropriate Itô correction term would appear. This indicates that the derivation of the stochastic thin-film equation in [33] relies on Stratonovich calculus and that the resulting model, as well as the one of [17], is naturally formulated with Stratonovich noise. In [33, Appendix C] it was then claimed that the specific choice of the stochastic calculus (Itô versus Stratonovich) is immaterial, at least in the case of space-time white noise.

In the present work we choose to consider the Stratonovich formulation of the thin-film equation due to two points: First, we prove that in Stratonovich formulation the construction of nonnegative martingale solutions is possible without an additional interface potential and allowing for touch down of solutions, thus relaxing the assumptions of $[13,19]$. Second, we show that the Stratonovich formulation allows for a simpler construction of solutions via a Trotter-Kato scheme. Notably, this scheme requires Stratonovich noise as only then the transport equation $(1.3 \mathrm{~b})$ is well-posed.

\subsection{Weak formulation and main result. Let}

$$
W(t, x):=\sum_{k \in \mathbb{Z}} \lambda_{k} \psi_{k}(x) \beta^{k}(t)
$$

where $\left(\lambda_{k}\right)_{k \in \mathbb{Z}}$ are real and nonnegative, define the family $\left(\psi_{k}\right)_{k \in \mathbb{Z}}$ through

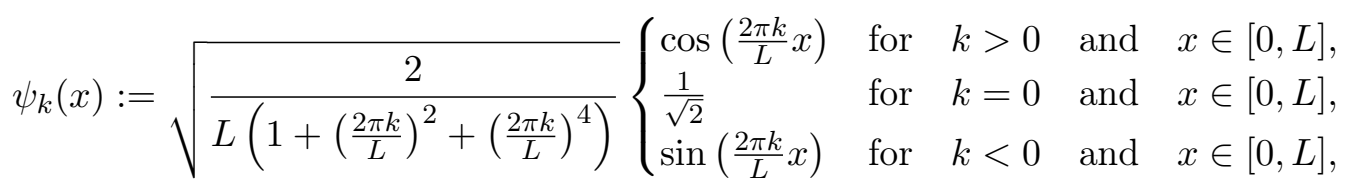

being an orthonormal basis of $H^{2}\left(\mathbb{T}_{L}\right)$ of eigenfunctions of the periodic Laplacian, and let $\left(\beta^{k}\right)_{k \in \mathbb{Z}}$ be a family of mutually independent standard real-valued $\left(\mathcal{F}_{t}\right)$-Wiener processes on a complete filtered probability space $\left(\Omega, \mathcal{F},\left(\mathcal{F}_{t}\right)_{t \in[0, T)}, \mathbb{P}\right)$, with a complete and right-continuous filtration $\left(\mathcal{F}_{t}\right)_{t \in[0, T)}$. From (1.8) it follows in particular

$$
\partial_{x} \psi_{k}=\frac{2 \pi k}{L} \psi_{-k} \quad \text { for } \quad k \in \mathbb{Z},
$$

so that

$$
\partial_{x}^{2} \psi_{k}=-\frac{4 \pi^{2} k^{2}}{L^{2}} \psi_{k}, \quad \partial_{x}^{3} \psi_{k}=-\frac{8 \pi^{3} k^{3}}{L^{3}} \psi_{-k}, \quad \partial_{x}^{4} \psi_{k}=\frac{16 \pi^{4} k^{4}}{L^{4}} \psi_{k} \quad \text { for } \quad k \in \mathbb{Z} .
$$

We will further assume the decay condition

$$
\sum_{k \in \mathbb{Z}} \lambda_{k}^{2}<\infty
$$

This ensures that $W$ takes values in $H^{2}\left(\mathbb{T}_{L}\right)$. Condition $(1.10)$ is the same as in $[19$, p. 417 , condition (H4)], taking into account that Fischer and Grün choose an orthonormal basis of $L^{2}\left(\mathbb{T}_{L}\right)$. 
Equation (1.1) with noise $W$ as in (1.7) may be rewritten using Itô calculus as (see [16, §3] for an analogous case)

$$
\mathrm{d} u=\partial_{x}\left(-\left(u^{2} \partial_{x}^{3} u\right)+\frac{1}{2} \sum_{k \in \mathbb{Z}} \lambda_{k}^{2} \psi_{k} \partial_{x}\left(\psi_{k} u\right)\right) \mathrm{d} t+\partial_{x}\left(\sum_{k \in \mathbb{Z}} \lambda_{k} \psi_{k} u \mathrm{~d} \beta^{k}\right)
$$

and its weak formulation is given by

$$
\begin{aligned}
\mathrm{d}(u, \varphi)_{2}= & \left(\int_{\{u(t, \cdot)>0\}} u^{2}\left(\partial_{x}^{3} u\right)\left(\partial_{x} \varphi\right) \mathrm{d} x-\frac{1}{2} \sum_{k \in \mathbb{Z}} \lambda_{k}^{2}\left(\left(\psi_{k} \partial_{x}\left(\psi_{k} u\right)\right), \partial_{x} \varphi\right)_{2}\right) \mathrm{d} t \\
& -\sum_{k \in \mathbb{Z}} \lambda_{k}\left(\psi_{k} u, \partial_{x} \varphi\right)_{2} \mathrm{~d} \beta^{k},
\end{aligned}
$$

$\mathbb{P}$-almost surely, for any $\varphi \in C^{\infty}\left(\mathbb{T}_{L}\right)$, where $(v, w)_{2}:=\int_{0}^{L} v(x) w(x) \mathrm{d} x$ for $v, w \in L^{2}\left(\mathbb{T}_{L}\right)$ denotes the inner product in $L^{2}\left(\mathbb{T}_{L}\right)$. Note that in the weak formulation, we only require the third derivative $\partial_{x}^{3} u$ to exist on the positivity set $\{u>0\}$ (cf. Definition 1.1).

We use the following notion of solutions:

Definition 1.1. Let $u_{0} \in H^{1}\left(\mathbb{T}_{L}\right)$ be nonnegative. A triple, consisting of a filtered probability space $\left(\tilde{\Omega}, \tilde{\mathcal{F}},\left(\tilde{\mathcal{F}}_{t}\right)_{t \in[0, T)}, \tilde{\mathbb{P}}\right)$, where $\left(\tilde{\mathcal{F}}_{t}\right)_{t \in[0, T)}$ is a complete and right-continuous filtration, an $\left(\tilde{\mathcal{F}}_{t}\right)$-adapted bounded continuous $H_{\mathrm{w}}^{1}\left(\mathbb{T}_{L}\right)$-valued process $\tilde{u}$ on $[0, T)$ such that the distributional derivative $\partial_{x}^{3} \tilde{u}$ is $\left(\tilde{\mathcal{F}}_{t}\right)$-adapted with $\partial_{x}^{3} \tilde{u} \in L^{2}(\{\tilde{u}>r\})$ for any $r>0$ and $\tilde{u}^{2}\left(\partial_{x}^{3} \tilde{u}\right) \in L^{2}(\{\tilde{u}>0\})$, $\tilde{\mathbb{P}}$-almost surely, as well as mutually independent standard real-valued $\left(\tilde{\mathcal{F}}_{t}\right)$-Wiener processes $\widetilde{\beta}^{k}$, is called a martingale solution of the stochastic thin-film equation (1.1) if its weak formulation

$$
\begin{aligned}
(\tilde{u}(t, \cdot), \varphi)_{2}-\left(u_{0}, \varphi\right)_{2}= & \int_{0}^{t} \int_{\left\{\tilde{u}\left(t^{\prime}, \cdot\right)>0\right\}} \tilde{u}^{2}\left(\partial_{x}^{3} \tilde{u}\right)\left(\partial_{x} \varphi\right) \mathrm{d} x \mathrm{~d} t^{\prime} \\
& -\frac{1}{2} \sum_{k \in \mathbb{Z}} \lambda_{k}^{2} \int_{0}^{t}\left(\psi_{k} \partial_{x}\left(\psi_{k} \tilde{u}\left(t^{\prime}, \cdot\right)\right), \partial_{x} \varphi\right)_{2} \mathrm{~d} t^{\prime} \\
& -\sum_{k \in \mathbb{Z}} \lambda_{k} \int_{0}^{t}\left(\psi_{k} \tilde{u}\left(t^{\prime}, \cdot\right), \partial_{x} \varphi\right)_{2} \mathrm{~d} \tilde{\beta}^{k}\left(t^{\prime}\right)
\end{aligned}
$$

is satisfied for every $\varphi \in C^{\infty}\left(\mathbb{T}_{L}\right)$ and $t \in[0, T), \tilde{\mathbb{P}}$-almost surely.

The main result of this work is

Theorem 1.2 (martingale solutions to the stochastic thin-film equation). Suppose that $u_{0} \in$ $H^{1}\left(\mathbb{T}_{L}\right)$ such that $u_{0} \geq 0$. Then, in the sense of Definition 1.1, there exists a martingale solution

$$
\left([0,1], \tilde{\mathcal{F}},\left(\tilde{\mathcal{F}}_{t}\right)_{t \in[0, T)}, \tilde{\mathbb{P}}\right), \quad \tilde{u}, \quad \text { and } \quad\left(\tilde{\beta}_{k}\right)_{k \in \mathbb{Z}}
$$

to the stochastic thin-film equation (1.1) for which $\tilde{u} \geq 0, \mathbb{P}$-almost surely, and for which the a-priori estimate

$$
\tilde{\mathbb{E}} \underset{t \in[0, T)}{\operatorname{ess}-\sup }\|\tilde{u}(t, \cdot)\|_{1,2}^{p} \leq C\left\|u_{0}\right\|_{1,2}^{p}
$$

is satisfied for any $p \in[2, \infty)$, where $C<\infty$ is independent of $\tilde{u}$ and $u_{0}$.

The proof of the above result is given in Section 5 below.

We emphasize once more that compared to the previous works $[13,19]$ we do not require an interface potential and that the occurrence of de-wetted regions with positive measure $\tilde{\mathbb{P}}|\{\tilde{u}(t, \cdot)=0\}|>0$ is included. This is due to the fact that the arguments of the present work only rely on controlling the energy $\frac{1}{2} \int_{0}^{L}\left(\partial_{x} \tilde{u}(t, x)\right)^{2} \mathrm{~d} x$ and not on controlling the entropy $\int_{0}^{L}|\ln \tilde{u}(t, x)| \mathrm{d} x$ as in $[13,19]$. 
Remark 1.3. Note that Theorem 1.2 easily translates to the case of random initial data $u_{0}$ satisfying

$$
u_{0} \in L^{q}\left(\Omega, \mathcal{F}_{0}, \mathbb{P} ; H^{1}\left(\mathbb{T}_{L}\right)\right) \quad \text { with } \quad u_{0} \geq 0, \quad \mathbb{P} \text {-almost surely }
$$

where $q \geq p$ is sufficiently large.

1.4. Decomposition of the dynamics. The idea of the construction is to split the dynamics of (1.12) into a deterministic evolution and a stochastic evolution; a Trotter-Kato-type decomposition that has been utilized in many other solution approaches for SPDEs, too. See for instance the works of Bensoussan, Glowinsky, and Răşcanu [3] and Gyöngy and Krylov [34] on the Zakaï equation or Govindan [30] for a mild-solution approach to semilinear stochastic evolution equations.

To begin with, we split the time interval $[0, T)$ into pieces of length $\delta:=\frac{T}{N+1}$, where $N \in \mathbb{N}_{0}$. Then we define

(D) Deterministic dynamics: On $[(j-1) \delta, j \delta)$ the function $v_{N}$ satisfies the evolution

$$
\left(v_{N}(t, \cdot), \varphi\right)_{2}-\left(v_{N}((j-1) \delta, \cdot), \varphi\right)_{2}=\int_{(j-1) \delta}^{t} \int_{\left\{v_{N}\left(t^{\prime},\right)>0\right\}} v_{N}^{2}\left(\partial_{x}^{3} v_{N}\right)\left(\partial_{x} \varphi\right) \mathrm{d} x \mathrm{~d} t^{\prime}
$$

for $t \in[(j-1) \delta, j \delta), \mathbb{P}$-almost surely, where $j=1, \ldots, N+1$ and $\varphi \in C^{\infty}\left(\mathbb{T}_{L}\right)$.

(S) Stochastic dynamics: On $[(j-1) \delta, j \delta)$ the function $w_{N}$ satisfies the evolution

$$
\begin{aligned}
\left(w_{N}(t, \cdot), \varphi\right)_{2}-\left(w_{N}((j-1) \delta, \cdot), \varphi\right)_{2}= & -\frac{1}{2} \sum_{k \in \mathbb{Z}} \lambda_{k}^{2} \int_{(j-1) \delta}^{t}\left(\psi_{k} \partial_{x}\left(\psi_{k} w_{N}\left(t^{\prime}, \cdot\right)\right), \partial_{x} \varphi\right)_{2} \mathrm{~d} t^{\prime} \\
& -\sum_{k \in \mathbb{Z}} \lambda_{k} \int_{(j-1) \delta}^{t}\left(\psi_{k} w_{N}\left(t^{\prime}, \cdot\right), \partial_{x} \varphi\right)_{2} \mathrm{~d} \beta^{k}\left(t^{\prime}\right)
\end{aligned}
$$

for $t \in[(j-1) \delta, j \delta), \mathbb{P}$-almost surely, where $j=1, \ldots, N+1$ and $\varphi \in C^{\infty}\left(\mathbb{T}_{L}\right)$.

(DS) Connecting deterministic and stochastic dynamics: We use

(1.13c) $\quad v_{N}(0, \cdot):=u_{0}, \quad v_{N}(j \delta, \cdot):=\lim _{t \nearrow j \delta} w_{N}(t, \cdot), \quad$ and $\quad w_{N}((j-1) \delta, \cdot):=\lim _{t \nearrow j \delta} v_{N}(t, \cdot)$,

$\mathbb{P}$-almost surely, where $j \in\{1, \ldots, N\}$.

Notice that (1.13a) is the weak formulation of (1.3a), while (1.13b) is the weak formulation of (1.3b), i.e., with noise $W$ as in (1.7),

$$
\mathrm{d} w_{N}=\frac{1}{2} \sum_{k \in \mathbb{Z}} \lambda_{k}^{2} \partial_{x}\left(\psi_{k} \partial_{x}\left(\psi_{k} w_{N}\right)\right) \mathrm{d} t+\sum_{k \in \mathbb{Z}} \lambda_{k} \partial_{x}\left(\psi_{k} w_{N}\right) \mathrm{d} \beta^{k} \quad \text { for } \quad t \in[(j-1) \delta, j \delta)
$$

and $j \in\{1, \ldots, N+1\}$. Since (1.3a) and (1.14) are in divergence form, they both automatically conserve mass $\int_{0}^{L} v_{N}(t, x) \mathrm{d} x$ or $\int_{0}^{L} w_{N}(t, x) \mathrm{d} x$, respectively.

Note that the dynamics in (D) are purely deterministic, while the dynamics in (S) are purely stochastic, with (DS) connecting them. In this work we show that solutions to (D) and (S) exist and that as $N \rightarrow \infty$, the scheme (D)-(S)-(DS) converges to a martingale solution to (1.1).

Note that the deterministic dynamics (D) are determined by the deterministic thin-film equation (1.3a), for which an existence theory of weak solutions due to Bernis and Friedman [6] is available. This theory has been subsequently upgraded to entropy-weak solutions by Beretta, Bertsch, and Dal Passo in [4] and Bertozzi and Pugh in [8] and to higher dimensions by Dal Passo, Garcke, and Grün in [14] and by Grün in [32]. The stochastic dynamics (S), on the other hand, are determined by a transport equation, to which we will apply a viscous regularization and the variational approach in order to construct solutions. While the existence of variational solutions is well-known (e.g. Krylov, Rozovskiu [44] and Gerencsér, Gyöngy, Krylov [21]), we recall some details on the proof in order to keep track on the dependency of the constants on the time step, as needed for the proof of convergence of the Trotter-Kato scheme. By construction, the scheme will preserve nonnegativity of solutions as long as we start with nonnegative and sufficiently regular initial data $u_{0}$, since this is known to be true for weak solutions to the deterministic 
thin-film equation (1.3a) (cf. [6, Theorem 4.1]), while (1.14) is a transport equation for which this assertion is not difficult to prove (cf. Proposition 3.3 below). Note, however, that the additional drift term in $(1.13 \mathrm{~b})$ is crucial in order to allow for the construction of solutions and that the dynamics (S) without this additional drift term are in fact not well-defined.

1.5. Outline. In $\S 2-4$ we prove that nonnegative solutions to the splitting scheme (D)-(S)(DS) exist such that certain bounds and regularity properties are satisfied. More precisely, in $\S 2$ we derive that solutions to the deterministic thin-film dynamics (D) (cf. Theorem 2.1 below and $[4,6,8]$ ) satisfy suitable bounds on the surface energy $\int_{0}^{L}\left(\partial_{x} v_{N}\right)^{2} \mathrm{~d} x$ (cf. Corollary 2.2 below). In $\S 3$ and Appendix A we move on to the stochastic dynamics (S) and prove that solutions exist by the vanishing viscosity method employing the variational approach (cf. Proposition A.2 and Proposition 3.2 below). The solution satisfies a bound on the expected surface energy $\mathbb{E} \int_{0}^{L}\left(\partial_{x} w_{N}\right)^{2} \mathrm{~d} x$ with suitable constants and we further prove that $w_{N}$ is, $\mathbb{P}$-almost surely, nonnegative (cf. Proposition 3.3 below). In $\S 4$ we summarize the results for the concatenated solution $u_{N}$ fulfilling (D)-(S)-(DS) (cf. Proposition 4.1 below) and prove additional regularity in time by cross interpolation (cf. Proposition 4.2 and Appendix B below).

In $\S 5$ we construct solutions to the original equation (1.1). The compactness argument in $\S 5.1$ is based on a generalization of Skorokhod's representation theorem due to Jakubowski (cf. Theorem 5.1 below and [38, Theorem 2]) by proving tightness in suitable spaces (cf. Proposition 5.2 below). The rest of $\S 5.1$ is devoted to the identification of the limits of the convergent subsequences (cf. Propositions 5.2, 5.3, and 5.5 below). In $\S 5.2$ we subsequently recover the stochastic thin-film equation (1.1) in the sense of Definition 1.1, leading to the main result, formulated in Theorem 1.2, in which nonnegative martingale solutions are obtained.

The paper is completed in $\S 6$ with concluding remarks on possible future directions.

\subsection{Notation and conventions.}

Sets. We write $\mathbb{N}:=\{1,2,3, \ldots\}$ for positive integers and $\mathbb{N}_{0}:=\mathbb{N} \cup\{0\}$. The set $\mathbb{T}_{L}$ denotes the torus of the interval $[0, L]$, where $L>0$. For sets $X$ and $K$ we write $K \Subset X$ if $K$ is a subset of $X(K \subseteq X)$ and $K$ is compact. We write $\mathbb{1}_{A}$ for the indicator function of a set $A \subseteq X$.

Lebesgue spaces. We denote by $L^{p}(\Omega, \mathcal{A}, \mu ; X)$ the Lebesgue spaces with $p \in[1, \infty]$ of functions $\Omega \rightarrow X$, where $\Omega$ is a set, $\mathcal{A}$ is a $\sigma$-algebra on $\Omega, \mu: \mathcal{A} \rightarrow[0, \infty]$ is a measure, and $X$ denotes a Banach space. In case that $\mathcal{A}$ denotes the Borel- $\sigma$-algebra and $\mu$ is the Lebesgue measure, we simply write $L^{p}(\Omega ; X)$, and if $X=\mathbb{R}$, we write $L^{p}(\Omega)$. We write $(u, v)_{2}:=\int_{0}^{L} u v \mathrm{~d} x$ and $\|u\|_{2}:=\sqrt{(u, u)_{2}}$ for the inner product and norm, where $u, v \in L^{2}\left(\mathbb{T}_{L}\right)$.

Hölder spaces and spaces of bounded continuous functions. For $\Omega \subseteq \mathbb{R}^{d}$ with $\partial \Omega \in C^{\infty}$, the space $C^{k+\alpha}(\Omega ; X)$ is the space of $k$-times differentiable functions $\Omega \rightarrow X$, where $k \in \mathbb{N}_{0}$, whose $k$-th derivatives are Hölder continuous with exponent $\alpha \in(0,1)$ on compact subsets of $\Omega$. For $k \in \mathbb{N}$ we write $C^{k-}(\Omega ; X)$ for the space of $(k-1)$-times differentiable functions $\Omega \rightarrow X$ whose $(k-1)$-th derivatives are Lipschitz continuous. We write $B C^{0}(\Omega ; X)$ for the space of bounded continuous functions $\Omega \rightarrow X$.

Sobolev(-Slobodeckij) spaces. Suppose that $\Omega \subseteq \mathbb{R}^{d}$ with $\partial \Omega \in C^{\infty}, s \in[0, \infty), p \in[1, \infty]$, and let $X$ be a Banach space. For a locally integrable function $u: \Omega \rightarrow X$ we define

$$
\|u\|_{W^{s, p}(\Omega ; X)}:=\left(\sum_{\alpha \in \mathbb{N}_{0}^{d}, 0 \leq|\alpha| \leq s} \int_{\Omega}\left\|\partial^{\alpha} u\right\|_{X}^{p} \mathrm{~d} x\right)^{\frac{1}{p}} \text { for } p \in[1, \infty) \quad \text { and } \quad s \in \mathbb{N}_{0},
$$

and $\|u\|_{W^{s, p}(\Omega ; X)}:=\|u\|_{W^{\lfloor s, p(\Omega ; X)}}+[u]_{W^{s, p}(\Omega ; X)}$ for $s \in(0, \infty) \backslash \mathbb{N}$, where

$$
[u]_{W^{s, p}(\Omega ; X)}:=\left(\sum_{\alpha \in \mathbb{N}_{0}^{d},|\alpha|=\lfloor s\rfloor} \int_{\Omega} \int_{\Omega} \frac{\left\|\partial^{\alpha} u(x)-\partial^{\alpha} u(y)\right\|_{X}^{p}}{|x-y|^{(s-\lfloor s\rfloor) p+d}} \mathrm{~d} x \mathrm{~d} y\right)^{\frac{1}{p}} \quad \text { for } \quad p \in[1, \infty),
$$


with the usual modifications for $p=\infty$. Then, the Sobolev-Slobodeckij space $W^{s, p}(\Omega ; X)$ is the space of all locally integrable $u: \Omega \rightarrow X$ such that $\|u\|_{W^{s, p}(\Omega ; X)}<\infty$. If $X=\mathbb{R}$, we simply write $W^{s, p}(\Omega)$. The space $W_{0}^{s, p}(\Omega ; X)$ is defined as the closure of $C_{\mathrm{c}}^{\infty}(\stackrel{\Omega}{\Omega} ; X)$ in $W^{s, p}(\Omega ; X)$. The space $W^{s, p}(\Omega ; X)$ for $s<0$ and $1 \leq p<\infty$ is defined as the dual of $W_{0}^{-s, p^{\prime}}\left(\Omega ; X^{\prime}\right)$, where $\frac{1}{p}+\frac{1}{p^{\prime}}=1$ and $X$ is reflexive.

Besov spaces. Assuming that $\Omega \subseteq \mathbb{R}^{d}$ with $\partial \Omega \in C^{\infty}, s \in \mathbb{R} \backslash \mathbb{Z}$, and $p, q \in[1, \infty)$, we introduce the Besov space $B_{q}^{s, p}(\Omega ; X):=\left(W^{\lfloor s\rfloor, p}(\Omega ; X), W^{\lceil s\rceil, p}(\Omega ; X)\right)_{\kappa, q}$, where $\kappa=s-\lfloor s\rfloor$ and $(\cdot, \cdot)_{\kappa, q}$ denotes the real interpolation functor. For $s \in \mathbb{Z}$ we define the Besov space $B_{q}^{s, p}(\Omega ; X):=$ $\left(W^{s-1, p}(\Omega ; X), W^{s+1, p}(\Omega ; X)\right)_{\frac{1}{2}, q}$. For an introduction to complex and real interpolation of operators, we refer to $[5, \S 3, \S 4]$ or $[56, \S 1]$, while Besov spaces with values in a Banach space are discussed for instance in [1] and [2, Chapter VII, §2].

Periodic Bessel-potential spaces. For $s \in[0, \infty)$ and $p \in(1, \infty)$ we define $H^{s, p}\left(\mathbb{T}_{L}\right)$ as the space of locally integrable $u: \mathbb{T}_{L} \rightarrow \mathbb{R}$ such that $\|u\|_{s, p}<\infty$, where for $p \neq 2$ we use

$$
\|u\|_{s, p}:=\left(\sum_{k \in \mathbb{Z}}\left(1+k^{2}\right)^{\frac{s p}{2}}|\hat{u}(k)|^{p}\right)^{\frac{1}{p}}, \quad \text { where } \hat{u}(k):=\frac{1}{\sqrt{L}} \int_{0}^{L} e^{\frac{2 \pi i k}{L} x} u(x) \mathrm{d} x
$$

and for $p=2$ we write $H^{s}\left(\mathbb{T}_{L}\right):=H^{s, 2}\left(\mathbb{T}_{L}\right)$ and define the inner products and norms by

$$
\begin{aligned}
(u, v)_{s, 2} & :=\sum_{j=0}^{s} \int_{0}^{L}\left(\partial_{x}^{j} u\right)\left(\partial_{x}^{j} v\right) \mathrm{d} x \quad \text { for } \quad s \in \mathbb{N}_{0}, \\
(u . v)_{s, 2} & :=\sum_{k \in \mathbb{Z}}\left(1+k^{2}\right)^{s}(\hat{u}(k))^{*} \hat{v}(k) \quad \text { for } \quad s \in(0, \infty) \backslash \mathbb{N}, \quad \hat{u}(k):=\frac{1}{\sqrt{L}} \int_{0}^{L} e^{\frac{2 \pi i k}{L} x} u(x) \mathrm{d} x,
\end{aligned}
$$

and $\|u\|_{s, 2}:=\sqrt{(u, u)_{s, 2}}$, where $u, v \in H^{s}\left(\mathbb{T}_{L}\right)$. We write $\dot{H}^{1}\left(\mathbb{T}_{L}\right)$ for the homogeneous Sobolev space of all locally integrable $u: \Omega \rightarrow \mathbb{R}$ with norm $\left\|\partial_{x} u\right\|_{2}<\infty$, where we identify $u, v \in$ $\dot{H}^{1}\left(\mathbb{T}_{L}\right)$, if $u-v$ is a constant. The space $H^{-s, p}\left(\mathbb{T}_{L}\right)$ is defined as the dual of $H^{s, p^{\prime}}\left(\mathbb{T}_{L}\right)$, where $\frac{1}{p}+\frac{1}{p^{\prime}}=1$. We write $\langle\cdot, \cdot\rangle$ or $\langle\langle\cdot, \cdot\rangle\rangle$ for the dual pairing of $H^{-1}\left(\mathbb{T}_{L}\right)$ with $H^{1}\left(\mathbb{T}_{L}\right)$ in $L^{2}\left(\mathbb{T}_{L}\right)$ or $L^{2}\left(\mathbb{T}_{L}\right)$ with $H^{2}\left(\mathbb{T}_{L}\right)$ in $H^{1}\left(\mathbb{T}_{L}\right)$, respectively. We write $H_{\mathrm{w}}^{1}\left(\mathbb{T}_{L}\right)$ for the space $H^{1}\left(\mathbb{T}_{L}\right)$ endowed with the weak topology induced by $\|\cdot\|_{1,2}$.

Periodic Besov spaces. For $s \in \mathbb{R} \backslash \mathbb{Z}, p \in(1, \infty)$, and $q \in[1, \infty)$, we define periodic Besov spaces by real interpolation as $B_{q}^{s, p}\left(\mathbb{T}_{L}\right):=\left(H^{\lfloor s\rfloor, p}\left(\mathbb{T}_{L}\right), H^{\lceil s\rceil, p}\left(\mathbb{T}_{L}\right)\right)_{\kappa, q}$, where $\kappa:=s-\lfloor s\rfloor$. For $s \in \mathbb{Z}$ we set $B_{q}^{s, p}\left(\mathbb{T}_{L}\right):=\left(H^{s+1, p}\left(\mathbb{T}_{L}\right), H^{s-1, p}\left(\mathbb{T}_{L}\right)\right)_{\frac{1}{2}, q}$. Periodic Besov spaces are investigated in detail in $[52, \S 3]$.

Hilbert-Schmidt operators. We denote by $L_{2}(U ; H)$ the space of Hilbert-Schmidt operators $U \rightarrow$ $H$, where $U$ and $H$ are separable Hilbert spaces, i.e., the space of bounded linear operators $B: U \rightarrow H$ with finite norm $\|B\|_{L_{2}(U ; H)}:=\sqrt{\sum_{k \in \mathbb{N}}\left\|B e_{k}\right\|_{H}^{2}}$, where $\left(e_{k}\right)_{k \in \mathbb{N}}$ denotes any orthonormal basis of $U$.

Probability spaces. We write $\mathbb{E}$ or $\tilde{\mathbb{E}}$ for the expectation with respect to a probability space $(\Omega, \mathcal{F}, \mathbb{P})$ or $(\tilde{\Omega}, \tilde{\mathcal{F}}, \tilde{\mathbb{P}})$, respectively. The symbol $\langle\cdot\rangle_{t}$ denotes the quadratic variation process. For probability spaces $(\Omega, \mathcal{F}, \mathbb{P})$ and $(\tilde{\Omega}, \tilde{\mathcal{F}}, \tilde{\mathbb{P}})$, and a topological space $(\mathcal{X}, \mathcal{T})$, suppose we are given random variables $X: \Omega \rightarrow \mathcal{X}$ and $\tilde{X}: \tilde{\Omega} \rightarrow \mathcal{X}$. Then we write $X \sim \tilde{X}$ and say that the laws of $X$ and $\tilde{X}$ coincide if $\mathbb{P}\{X \in \mathcal{U}\}=\tilde{\mathbb{P}}\{\tilde{X} \in \mathcal{U}\}$ for every $\mathcal{U} \in \mathcal{T}$.

Constants. In what follows, $c, C, c_{j}$, and $C_{j}$ will denote generic positive and finite constants and if deemed necessary, their (in-)dependence on parameters or functions is specified. 


\section{Deterministic DYNAmics}

Consider the deterministic thin-film dynamics (1.3a), i.e.,

$$
\partial_{t} v=-\partial_{x}\left(v^{2} \partial_{x}^{3} v\right) \quad \text { on } \quad[0, \delta) .
$$

We use the existence and regularity results on solutions to (2.1) developed in $[4,8]$ as the proof of non-negativity therein does not require the use of the entropy as in [6]. Note that Beretta, Bertsch, and Dal Passo in [6] consider solutions to (2.1) on the interval $[0, L]$ but with homogeneous (Neumann) data

$$
\partial_{x} v(\cdot, 0)=\partial_{x} v(\cdot, L)=0 \quad \text { and } \quad\left(v^{n} \partial_{x}^{3} v\right)(\cdot, 0)=\left(v^{n} \partial_{x}^{3} v\right)(\cdot, L)=0,
$$

though the construction of solutions on the torus $\mathbb{T}_{L}$ works in the same manner. The following statements form a summary of those in [4, Proposition 1.1] and [8, Theorem 2.1, Proposition 4.6, Proposition 4.8].

Theorem 2.1 (Beretta, Bertsch, Dal Passo [4], Bertozzi and Pugh [8]). Assume that $v_{0} \in$ $H^{1}\left(\mathbb{T}_{L}\right)$ with $v_{0} \geq 0$. Then, there exists a function $v:[0, \delta) \times \mathbb{T}_{L} \rightarrow[0, \infty)$ with the following properties:

(a) $v \in C^{\frac{1}{8}}, \frac{1}{2}\left([0, \delta] \times \mathbb{T}_{L}\right)$ (mixed Hölder continuity with exponent $\frac{1}{8}$ in time and $\frac{1}{2}$ in space).

(b) Initial value: $v(0, \cdot)=v_{0}$ in the sense that $\left\|v(t, \cdot)-v_{0}\right\|_{1,2} \rightarrow 0$ as $t \searrow 0$.

(c) $v \in L^{\infty}\left([0, \delta) ; H^{1}\left(\mathbb{T}_{L}\right)\right)$.

(d) $v^{2} \partial_{x}^{3} v \in L^{2}(\{v>0\})$.

(e) Mass conservation: $\int_{0}^{L} v \mathrm{~d} x=\int_{0}^{L} v_{0} \mathrm{~d} x$ on the time interval $[0, \delta)$.

(f) The function $v$ satisfies

$$
\int_{0}^{\delta} \int_{0}^{L} v\left(\partial_{t} \phi\right) \mathrm{d} x \mathrm{~d} t+\int_{0}^{\delta} \int_{\{v(t, \cdot)>0\}} v^{2}\left(\partial_{x}^{3} v\right)\left(\partial_{x} \phi\right) \mathrm{d} x \mathrm{~d} t=0
$$

for all $\phi \in C_{\mathrm{c}}^{\infty}\left((0, \delta) ; C^{\infty}\left(\mathbb{T}_{L}\right)\right)$.

In addition to mass conservation, we also need a quantitative energy estimate, which essentially follows from the construction of $[4,6,8]$ :

Corollary 2.2 (quantitative estimate). In the situation of Theorem 2.1 there exists a solution $v:[0, \delta) \times \mathbb{T}_{L} \rightarrow[0, \infty)$ satisfying the properties (a)-(f) and further

$$
\left\|\partial_{x} v(t, \cdot)\right\|_{2}^{p}+2 \int_{0}^{t}\left\|\partial_{x} v\left(t^{\prime}, \cdot\right)\right\|_{2}^{p-2} \int_{\left\{v\left(t^{\prime}, \cdot\right)>0\right\}}\left(v\left(t^{\prime}, x\right)\right)^{2}\left(\partial_{x}^{3} v\left(t^{\prime}, x\right)\right)^{2} \mathrm{~d} x \mathrm{~d} t^{\prime} \leq\left\|\partial_{x} v_{0}\right\|_{2}^{p}
$$

for $t \in[0, \delta)$, where $p \in[2, \infty)$ is arbitrary.

Proof of Corollary 2.2. Denote by $v^{\varepsilon}$ unique classical solutions to the approximating problems

$$
\partial_{t} v^{\varepsilon}+\partial_{x}\left(f_{\varepsilon}\left(v^{\varepsilon}\right) \partial_{x}^{3} v^{\varepsilon}\right)=0 \quad \text { in } \quad[0, \delta) \times \mathbb{T}_{L},
$$

with $f_{\varepsilon}(s)=\frac{s^{4}}{\varepsilon+s^{2}}$ and initial data $v_{0}^{\varepsilon} \in C^{\infty}\left(\mathbb{T}_{L}\right)$ such that $v_{0}^{\varepsilon}>0$ and $\left\|v_{0}-v_{0}^{\varepsilon}\right\|_{1,2} \rightarrow 0$ as $\varepsilon \searrow 0$ (cf. $[4, \S 1]$ for details). From [4, Eq. (1.8)] we infer that

$$
\left\|\partial_{x} v^{\varepsilon}(t, \cdot)\right\|_{2}^{2}+2 \int_{0}^{t} \int_{\left\{v^{\varepsilon}\left(t^{\prime}, \cdot\right)>0\right\}} f_{\varepsilon}\left(v^{\varepsilon}\right)\left(\partial_{x}^{3} v^{\varepsilon}\right)^{2} \mathrm{~d} x \mathrm{~d} t^{\prime}=\left\|\partial_{x} v_{0}^{\varepsilon}\right\|_{2}^{2} \quad \text { for all } \quad t \in[0, \delta)
$$

holds true. Since as $\varepsilon \searrow 0$ a subsequence of $v^{\varepsilon}$ uniformly converges to $v$ of Theorem 2.1 (cf. [4, (1.13)]), for any $r>0$ and $\varepsilon>0$ sufficiently small such that $\sup _{(t, x) \in[0, \delta) \times \mathbb{T}_{L}}\left|v^{\varepsilon}(t, x)-v(t, x)\right| \leq$ $\frac{r}{2}$, we have

$$
\int_{0}^{\delta} \int_{\{v(t, \cdot)>r\}}\left(\partial_{x}^{3} v^{\varepsilon}\right)^{2} \mathrm{~d} x \mathrm{~d} t \leq \frac{1}{f_{\varepsilon}(r / 2)} \int_{0}^{\delta} \int_{\{v(t, \cdot)>r\}} f_{\varepsilon}\left(v^{\varepsilon}\right)\left(\partial_{x}^{3} v^{\varepsilon}\right)^{2} \mathrm{~d} x \mathrm{~d} t \stackrel{(2.4)}{\leq} \frac{8 \varepsilon+2 r^{2}}{r^{4}}\left\|\partial_{x} v_{0}\right\|_{2}^{2} .
$$


A diagonal sequence argument implies that, up to taking another subsequence, we have for some $\zeta \in L^{2}\left(\mathbb{T}_{L}\right)$

$$
\int_{0}^{\delta} \int_{\{v(t, \cdot)>r\}} \eta \partial_{x}^{3} v^{\varepsilon} \mathrm{d} x \mathrm{~d} t \rightarrow \int_{0}^{\delta} \int_{\{v(t, \cdot)>r\}} \eta \zeta \mathrm{d} x \mathrm{~d} t \quad \text { as } \quad \varepsilon \searrow 0
$$

for any $r>0$ and any $\eta \in C_{\mathrm{c}}^{\infty}(\{v>r\})$. On the other hand, through integration by parts and bounded convergence

$$
\int_{0}^{\delta} \int_{\{v(t, \cdot)>r\}} \eta\left(\partial_{x}^{3} v^{\varepsilon}\right) \mathrm{d} x \mathrm{~d} t=-\int_{0}^{\delta} \int_{\{v(t, \cdot)>r\}}\left(\partial_{x}^{3} \eta\right) v^{\varepsilon} \mathrm{d} x \mathrm{~d} t \rightarrow-\int_{0}^{\delta} \int_{\{v(t, \cdot)>r\}}\left(\partial_{x}^{3} \eta\right) v \mathrm{~d} x \mathrm{~d} t,
$$

as $\varepsilon \searrow 0$, i.e., $\zeta=\partial_{x}^{3} v$. From (2.4) we deduce that, up to taking another subsequence, also estimate (2.3) is valid for $p=2$ by weak lower-semicontinuity using $\partial_{x}^{3} v^{\varepsilon} \rightarrow \partial_{x}^{3} v$ in $L^{2}(\{v>0\})$ and $\sup _{(t, x) \in[0, \delta) \times \mathbb{T}_{L}}\left|v^{\varepsilon}(t, x)-v(t, x)\right| \rightarrow 0$ as $\varepsilon \searrow 0$. Estimate (2.3) for $p \in[2, \infty)$ follows from the one for $p=2$ by noting that

$$
\begin{aligned}
& \left\|\partial_{x} v(t, \cdot)\right\|_{2}^{p}+\int_{0}^{t}\left\|\partial_{x} v\left(t^{\prime}, \cdot\right)\right\|_{2}^{p-2} \int_{\left\{v\left(t^{\prime}, \cdot\right)>0\right\}}\left(v\left(t^{\prime}, x\right)\right)^{2}\left(\partial_{x}^{3} v\left(t^{\prime}, x\right)\right)^{2} \mathrm{~d} x \mathrm{~d} t^{\prime} \\
& \leq \sup _{t^{\prime} \in[0, t]}\left\|\partial_{x} v\left(t^{\prime}, \cdot\right)\right\|_{2}^{p-2}\left(\left\|\partial_{x} v(t, \cdot)\right\|_{2}^{2}+\int_{0}^{t} \int_{\left\{v\left(t^{\prime}, \cdot\right)>0\right\}}\left(v\left(t^{\prime}, x\right)\right)^{2}\left(\partial_{x}^{3} v\left(t^{\prime}, x\right)\right)^{2} \mathrm{~d} x \mathrm{~d} t^{\prime}\right) \\
& \leq\left\|\partial_{x} v_{0}\right\|_{2}^{p-2}\left(\left\|\partial_{x} v(t, \cdot)\right\|_{2}^{2}+\int_{0}^{t} \int_{\left\{v\left(t^{\prime}, \cdot\right)>0\right\}}\left(v\left(t^{\prime}, x\right)\right)^{2}\left(\partial_{x}^{3} v\left(t^{\prime}, x\right)\right)^{2} \mathrm{~d} x \mathrm{~d} t^{\prime}\right) \leq\left\|\partial_{x} v_{0}\right\|_{2}^{p} .
\end{aligned}
$$

\section{Stochastic DYNAmics}

Denote by $\left(\Omega, \mathcal{F},\left(\mathcal{F}_{t}\right)_{t \in[0, \delta]}, \mathbb{P}\right)$ a complete filtered probability space such that the filtration $\left(\mathcal{F}_{t}\right)_{t \in[0, \delta]}$ is complete and right-continuous. Further denote by $\left(\beta^{k}\right)_{k \in \mathbb{Z}}$ mutually independent standard real-valued $\left(\mathcal{F}_{t}\right)$-Wiener processes. Our aim is to construct weak solutions to equation (1.14), i.e.,

$$
\mathrm{d} w=\frac{1}{2} \sum_{k \in \mathbb{Z}} \lambda_{k}^{2} \partial_{x}\left(\psi_{k} \partial_{x}\left(\psi_{k} w\right)\right) \mathrm{d} t+\sum_{k \in \mathbb{Z}} \lambda_{k} \partial_{x}\left(\psi_{k} w\right) \mathrm{d} \beta^{k} \quad \text { on } \quad[0, \delta),
$$

satisfying suitable bounds. The material leading to Proposition 3.2 is standard (see for instance $[21,44])$ and given in Appendix A. There, we present some additional details in order to track the dependency of the occurring constants on the time step, which will be needed below.

We introduce the operator

$$
A^{0}: H^{1}\left(\mathbb{T}_{L}\right) \rightarrow H^{-1}\left(\mathbb{T}_{L}\right), \quad w \mapsto \frac{1}{2} \sum_{k \in \mathbb{Z}} \lambda_{k}^{2} \partial_{x}\left(\psi_{k} \partial_{x}\left(\psi_{k} w\right)\right)
$$

and the diagonal Hilbert-Schmidt-valued operator

$$
B^{0}: H^{1}\left(\mathbb{T}_{L}\right) \rightarrow L_{2}\left(H^{2}\left(\mathbb{T}_{L}\right) ; L^{2}\left(\mathbb{T}_{L}\right)\right), \quad w \mapsto\left(v \mapsto \sum_{k \in \mathbb{Z}} \lambda_{k}\left(v, \psi_{k}\right)_{2,2}\left(\partial_{x}\left(\psi_{k} w\right)\right)\right) .
$$

Equation (3.1) now attains the abstract form

$$
\partial_{t} w=A^{0} w+\left(B^{0} w\right) \mathrm{d} W_{H^{2}\left(\mathbb{T}_{L}\right)}, \quad \text { where } \quad W_{H^{2}\left(\mathbb{T}_{L}\right)}:=\sum_{k \in \mathbb{Z}} \beta^{k} \psi_{k} .
$$

Note that $W_{H^{2}\left(\mathbb{T}_{L}\right)}$ is a cylindrical $\left(\mathcal{F}_{t}\right)$-Wiener process in $H^{2}\left(\mathbb{T}_{L}\right)$ with $\left(B^{0} w\right) \mathrm{d} W_{H^{2}\left(\mathbb{T}_{L}\right)}=$ $\partial_{x}(w \circ \mathrm{d} W)$ for any $w \in H^{1}\left(\mathbb{T}_{L}\right)$, where $W$ is as in (1.7). We introduce the concept of weak solutions to (3.4): 
Definition 3.1. A weak solution to (3.4) is a continuous $\left(\mathcal{F}_{t}\right)$-adapted $L^{2}\left(\mathbb{T}_{L}\right)$-valued process $w$ such that its $\mathrm{d} t \otimes \mathrm{d} \mathbb{P}$-equivalence class $\hat{w}$ meets

$$
\hat{w} \in L^{2}\left([0, \delta) \times \Omega, \mathrm{d} t \otimes \mathrm{d} \mathbb{P} ; H^{1}\left(\mathbb{T}_{L}\right)\right)
$$

and $\mathbb{P}$-almost surely

$$
w(t, \cdot)=w_{0}+\int_{0}^{t} A^{0} \bar{w}\left(t^{\prime}, \cdot\right) \mathrm{d} t^{\prime}+\int_{0}^{t}\left(B^{0} \bar{w}\left(t^{\prime}, \cdot\right)\right) \mathrm{d} W_{H^{2}\left(\mathbb{T}_{L}\right)}\left(t^{\prime}, \cdot\right) \quad \text { for } \quad t \in[0, \delta),
$$

where $\bar{w}$ denotes any $H^{1}\left(\mathbb{T}_{L}\right)$-valued progressively measurable $\mathrm{d} t \otimes \mathrm{d} \mathbb{P}$-version of $\hat{w}$.

With help of Proposition A.2 we can show:

Proposition 3.2. Suppose that $p \in[2, \infty)$ and let (1.10) hold true. Then, for any

$$
w_{0} \in L^{p}\left(\Omega, \mathcal{F}_{0}, \mathbb{P} ; H^{1}\left(\mathbb{T}_{L}\right)\right)
$$

there exists a solution $w$ of (3.1) with initial data $w_{0}$ satisfying the a-priori estimates

$$
\begin{aligned}
& \underset{t \in[0, \delta)}{\mathbb{E} \text { ess-sup }}\|w(t, \cdot)\|_{1,2}^{p} \leq C_{1} \mathbb{E}\left\|w_{0}\right\|_{1,2}^{p}, \\
& \underset{t \nearrow \delta}{\limsup } \mathbb{E}\left\|\partial_{x} w(t, \cdot)\right\|_{2}^{p} \leq e^{C_{2} \delta}\left(\mathbb{E}\left\|\partial_{x} w_{0}\right\|_{2}^{p}+C_{3} \delta \mathbb{E}\left|\int_{0}^{L} w_{0} \mathrm{~d} x\right|^{p}\right),
\end{aligned}
$$

where $C_{1}, C_{2}, C_{3}<\infty$ are independent of $\delta, w$, and $w_{0}$. Furthermore, the mass is conserved, i.e., $\int_{0}^{L} w(t, \cdot) \mathrm{d} x=\int_{0}^{L} w_{0} \mathrm{~d} x$ holds true for $t \in[0, \delta), \mathbb{P}$-almost surely.

Proof of Proposition 3.2. Suppose that $w^{\varepsilon}$ is the unique variational solution to the regularized equation (A.1) below, i.e.,

$$
\mathrm{d} w^{\varepsilon}=\left(\frac{1}{2} \sum_{k \in \mathbb{Z}} \lambda_{k}^{2} \partial_{x}\left(\psi_{k} \partial_{x}\left(\psi_{k} w^{\varepsilon}\right)\right)+\varepsilon \partial_{x}^{2} w^{\varepsilon}\right) \mathrm{d} t+\sum_{k \in \mathbb{Z}} \lambda_{k} \partial_{x}\left(\psi_{k} w^{\varepsilon}\right) \mathrm{d} \beta^{k} \quad \text { on } \quad[0, \delta),
$$

given by Proposition A.2 below. Since the bound (A.8a) of Proposition A.2 is satisfied uniformly in $\varepsilon$, by weak-* sequential compactness of $L^{p}\left(\Omega, \mathcal{F}, \mathbb{P} ; L^{\infty}\left([0, \delta) ; H^{1}\left(\mathbb{T}_{L}\right)\right)\right)$, we may take a subsequence, again denoted by $w^{\varepsilon}$, that weak-*-converges to a limit function $w \in$ $L^{p}\left(\Omega, \mathcal{F}, \mathbb{P} ; L^{\infty}\left([0, \delta) ; H^{1}\left(\mathbb{T}_{L}\right)\right)\right)$. Testing (A.6) of Definition A.1 below with $\varphi \in C^{\infty}\left(\mathbb{T}_{L}\right)$ gives

$$
\begin{aligned}
\left(w^{\varepsilon}(t, \cdot), \varphi\right)_{2}= & \left(w_{0}, \varphi\right)_{2}-\frac{1}{2} \sum_{k \in \mathbb{Z}} \lambda_{k}^{2} \int_{0}^{t}\left(\psi_{k} \partial_{x}\left(\psi_{k} \bar{w}^{\varepsilon}\left(t^{\prime}, \cdot\right)\right), \partial_{x} \varphi\right)_{2} \mathrm{~d} t^{\prime} \\
& -\varepsilon \int_{0}^{t}\left(\partial_{x} w^{\varepsilon}\left(t^{\prime}, \cdot\right), \partial_{x} \varphi\right)_{2} \mathrm{~d} t^{\prime} \\
& -\sum_{k \in \mathbb{Z}} \lambda_{k} \int_{0}^{t}\left(\psi_{k} \bar{w}^{\varepsilon}\left(t^{\prime}, \cdot\right), \partial_{x} \varphi\right)_{2} \mathrm{~d} \beta^{k}\left(t^{\prime}\right) \quad \text { for } \quad t \in[0, \delta), \quad \mathbb{P} \text {-almost surely. }
\end{aligned}
$$

Now, we argue as in $\left[49\right.$, Proof of Theorem 4.2.4], i.e., we test against $\eta \in L^{\infty}\left(\Omega, \mathcal{F}, \mathbb{P} ; L^{\infty}([0, \delta))\right)$ and pass to the limit as $\varepsilon \searrow 0$, so that

$$
\begin{aligned}
\mathbb{E} \int_{0}^{\delta}(w(t, \cdot), \varphi)_{2} \eta(t) \mathrm{d} t= & \mathbb{E} \int_{0}^{\delta}\left(w_{0}, \varphi\right)_{2} \eta(t) \mathrm{d} t \\
& -\frac{1}{2} \sum_{k \in \mathbb{Z}} \lambda_{k}^{2} \mathbb{E} \int_{0}^{\delta} \int_{0}^{t}\left(\psi_{k} \partial_{x}\left(\psi_{k} \bar{w}\left(t^{\prime}, \cdot\right)\right), \partial_{x} \varphi\right)_{2} \mathrm{~d} t^{\prime} \eta(t) \mathrm{d} t \\
& -\sum_{k \in \mathbb{Z}} \lambda_{k} \mathbb{E} \int_{0}^{\delta} \int_{0}^{t}\left(\psi_{k} \bar{w}\left(t^{\prime}, \cdot\right), \partial_{x} \varphi\right)_{2} \mathrm{~d} \beta^{k}\left(t^{\prime}\right) \eta(t) \mathrm{d} t
\end{aligned}
$$


Since the limiting equation (3.7) holds true for all test functions $\eta \in L^{\infty}\left(\Omega, \mathcal{F}, \mathbb{P} ; L^{\infty}([0, \delta))\right)$, it is true almost everywhere in $(\omega, t) \in \Omega \times[0, \delta)$. Next, as in [49, Proof of Theorem 4.2.4], we re-define $w$ by the right-hand side of the limiting equation (3.7), so that

$$
\begin{aligned}
(w(t, \cdot), \varphi)_{2}= & \left(w_{0}, \varphi\right)_{2}-\frac{1}{2} \sum_{k \in \mathbb{Z}} \lambda_{k}^{2} \int_{0}^{t}\left(\psi_{k} \partial_{x}\left(\psi_{k} \bar{w}\left(t^{\prime}, \cdot\right)\right), \partial_{x} \varphi\right)_{2} \mathrm{~d} t^{\prime} \\
& -\sum_{k \in \mathbb{Z}} \lambda_{k} \int_{0}^{t}\left(\psi_{k} \bar{w}\left(t^{\prime}, \cdot\right), \partial_{x} \varphi\right)_{2} \mathrm{~d} \beta^{k}\left(t^{\prime}\right) \quad \text { for } t \in[0, \delta), \quad \mathbb{P} \text {-almost surely. }
\end{aligned}
$$

Hence, (3.5) is indeed satisfied and the initial value $\left.w\right|_{t=0}=w_{0}$ holds true in $H^{-1}\left(\mathbb{T}_{L}\right), \mathbb{P}$-almost surely. Taking $\varphi=1$ in (3.8) implies conservation of mass, i.e., $\int_{0}^{L} w(t, \cdot) \mathrm{d} x=\int_{0}^{L} w_{0} \mathrm{~d} x$ holds true for $t \in[0, \delta), \mathbb{P}$-almost surely. Furthermore, uniformity of estimates (A.8) in $\varepsilon$ together with weak lower-semicontinuity of the norms and mass conservation imply that estimates (3.6) hold true. Finally, it is immediate to notice that from (A.6) of Definition A.1 below it follows

$$
\begin{aligned}
w_{0} & \in L^{2}\left(\Omega, \mathcal{F}, \mathbb{P} ; L^{2}\left(\mathbb{T}_{L}\right)\right), \\
\left(t \mapsto A^{0} \bar{w}(t, \cdot)\right) & \in L^{2}\left([0, \delta) \times \Omega, \mathrm{d} t \otimes \mathrm{d} \mathbb{P} ; H^{-1}\left(\mathbb{T}_{L}\right)\right), \\
\left(t \mapsto B^{0} \bar{w}(t, \cdot)\right) & \in L^{2}\left([0, \delta) \times \Omega, \mathrm{d} t \otimes \mathrm{d} \mathbb{P} ; L_{2}\left(H^{2}\left(\mathbb{T}_{L}\right) ; L^{2}\left(\mathbb{T}_{L}\right)\right)\right), \\
\hat{w} & \in L^{2}\left([0, \delta) \times \Omega, \mathrm{d} t \otimes \mathrm{d} \mathbb{P} ; H^{1}\left(\mathbb{T}_{L}\right)\right),
\end{aligned}
$$

so that $w$ is a continuous $\left(\mathcal{F}_{t}\right)$-adapted $L^{2}\left(\mathbb{T}_{L}\right)$-valued process by [49, Theorem 4.2.5].

We can furthermore show nonnegativity of weak solutions to (3.1):

Proposition 3.3 (nonnegativity). In the situation of Proposition 3.2 assume $w_{0} \geq 0, \mathbb{P}$-almost surely. Then, we have $w \geq 0, \mathbb{P}$-almost surely.

Proof of Proposition 3.3. We first introduce suitable regular entropies. Therefore, we take $\eta_{\varepsilon}(s):=$ $\eta(s / \varepsilon)$ for $s \in \mathbb{R}$ and $\varepsilon>0$, where $\eta \in C^{\infty}(\mathbb{R})$ with $0 \leq \eta \leq 1,\left.\eta\right|_{(-\infty,-2]}=1$, and $\left.\eta\right|_{\mathbb{R} \backslash[-1, \infty)}=0$. We define

$$
\Gamma_{\varepsilon}(s):=-s \eta_{\varepsilon}(s), \quad \text { where } \quad \varepsilon>0 \quad \text { and } \quad s \in \mathbb{R}
$$

and consider the entropy functional

$$
L^{2}\left(\mathbb{T}_{L}\right) \rightarrow \mathbb{R}, \quad \varphi \mapsto \int_{0}^{L} \Gamma_{\varepsilon}(\varphi(x)) \mathrm{d} x
$$

Applying Itô's lemma in form of [45, Theorem 3.1], one may verify conditions [45, §3 (i)-(iv)], which is done in $[45, \S 4]$. As a result, we obtain

$$
\begin{aligned}
\int_{0}^{L} \Gamma_{\varepsilon}(w(t, \cdot)) \mathrm{d} x= & \int_{0}^{L} \Gamma_{\varepsilon}\left(w_{0}\right) \mathrm{d} x+\sum_{k \in \mathbb{Z}} \lambda_{k} \int_{0}^{t} \int_{0}^{L}\left(\partial_{s} \Gamma_{\varepsilon}\right)(w)\left(\partial_{x}\left(\psi_{k} w\right)\right) \mathrm{d} x \mathrm{~d} \beta^{k} \\
& -\frac{1}{2} \sum_{k \in \mathbb{Z}} \lambda_{k}^{2} \int_{0}^{t} \int_{0}^{L}\left(\partial_{s}^{2} \Gamma_{\varepsilon}\right)(w)\left(\partial_{x} w\right) \psi_{k}\left(\partial_{x}\left(\psi_{k} w\right)\right) \mathrm{d} x \mathrm{~d} t^{\prime} \\
& +\frac{1}{2} \sum_{k \in \mathbb{Z}} \lambda_{k}^{2} \int_{0}^{t} \int_{0}^{L}\left(\partial_{s}^{2} \Gamma_{\varepsilon}\right)(w)\left(\partial_{x}\left(\psi_{k} w\right)\right)^{2} \mathrm{~d} x \mathrm{~d} t^{\prime}, \quad \mathbb{P} \text {-almost surely. }
\end{aligned}
$$


We further simplify the second line and obtain

$$
\begin{aligned}
\int_{0}^{L} & \left(\partial_{s}^{2} \Gamma_{\varepsilon}\right)(w)\left(\partial_{x} w\right) \psi_{k}\left(\partial_{x}\left(\psi_{k} w\right)\right) \mathrm{d} x \\
= & \int_{0}^{L}\left(\partial_{s}^{2} \Gamma_{\varepsilon}\right)(w)\left(\partial_{x}\left(\psi_{k} w\right)\right)^{2} \mathrm{~d} x-\int_{0}^{L}\left(\partial_{s}^{2} \Gamma_{\varepsilon}\right)(w) w\left(\partial_{x} \psi_{k}\right)\left(\partial_{x}\left(\psi_{k} w\right)\right) \mathrm{d} x \\
= & \int_{0}^{L}\left(\partial_{s}^{2} \Gamma_{\varepsilon}\right)(w)\left(\partial_{x}\left(\psi_{k} w\right)\right)^{2} \mathrm{~d} x-\int_{0}^{L}\left(\partial_{s}^{2} \Gamma_{\varepsilon}\right)(w) w^{2}\left(\partial_{x} \psi_{k}\right)^{2} \mathrm{~d} x \\
& -\frac{1}{2} \int_{0}^{L}\left(\partial_{s}^{2} \Gamma_{\varepsilon}\right)(w) w\left(\partial_{x} w\right)\left(\partial_{x} \psi_{k}^{2}\right) \mathrm{d} x \\
= & \int_{0}^{L}\left(\partial_{s}^{2} \Gamma_{\varepsilon}\right)(w)\left(\partial_{x}\left(\psi_{k} w\right)\right)^{2} \mathrm{~d} x-\int_{0}^{L}\left(s^{2} \partial_{s}^{2} \Gamma_{\varepsilon}\right)(w)\left(\partial_{x} \psi_{k}\right)^{2} \mathrm{~d} x+\frac{1}{2} \int_{0}^{L} \bar{\Gamma}_{\varepsilon}(w)\left(\partial_{x}^{2} \psi_{k}^{2}\right) \mathrm{d} x,
\end{aligned}
$$

$\mathbb{P}$-almost surely, where we have defined

$$
\bar{\Gamma}_{\varepsilon}(s):=\int_{s}^{0}\left(s \partial_{s}^{2} \Gamma_{\varepsilon}\right)\left(s_{1}\right) \mathrm{d} s_{1} .
$$

This implies

$$
\begin{aligned}
\int_{0}^{L} \Gamma_{\varepsilon}(w(t, \cdot)) \mathrm{d} x= & \int_{0}^{L} \Gamma_{\varepsilon}\left(w_{0}\right) \mathrm{d} x+\sum_{k \in \mathbb{Z}} \lambda_{k} \int_{0}^{t} \int_{0}^{L}\left(\partial_{s} \Gamma_{\varepsilon}\right)(w) \partial_{x}\left(\psi_{k} w\right) \mathrm{d} x \mathrm{~d} \beta^{k} \\
& +\frac{1}{2} \sum_{k \in \mathbb{Z}} \lambda_{k}^{2} \int_{0}^{t} \int_{0}^{L}\left(s^{2} \partial_{s}^{2} \Gamma_{\varepsilon}\right)(w)\left(\partial_{x} \psi_{k}\right)^{2} \mathrm{~d} x \mathrm{~d} t^{\prime} \\
& -\frac{1}{4} \sum_{k \in \mathbb{Z}} \lambda_{k}^{2} \int_{0}^{t} \int_{0}^{L} \bar{\Gamma}_{\varepsilon}(w)\left(\partial_{x}^{2} \psi_{k}^{2}\right) \mathrm{d} x \mathrm{~d} t^{\prime}, \quad \mathbb{P} \text {-almost surely. }
\end{aligned}
$$

Next, we recognize that

$$
\begin{aligned}
s^{2} \partial_{s}^{2} \Gamma_{\varepsilon}(s) & =-2 s\left(s \partial_{s} \eta_{\varepsilon}\right)(s)-s\left(s^{2} \partial_{s}^{2} \eta_{\varepsilon}\right)(s) \leq \varepsilon\left(2 \sup _{s \in \mathbb{R}}\left|s \partial_{s} \eta(s)\right|+\sup _{s \in \mathbb{R}}\left|s^{2} \partial_{s}^{2} \eta(s)\right|\right), \\
\left|\bar{\Gamma}_{\varepsilon}(s)\right| & =\left|\int_{s}^{0}\left(2\left(s \partial_{s} \eta_{\varepsilon}\right)\left(s_{1}\right)+\left(s^{2} \partial_{s}^{2} \eta_{\varepsilon}\right)\left(s_{1}\right)\right) \mathrm{d} s_{1}\right| \leq \varepsilon\left(2 \sup _{s \in \mathbb{R}}\left|s \partial_{s} \eta(s)\right|+\varepsilon \sup _{s \in \mathbb{R}}\left|s^{2} \partial_{s}^{2} \eta(s)\right|\right),
\end{aligned}
$$

where we have used $\operatorname{supp}\left(s^{2} \partial_{s}^{2} \eta_{\varepsilon}\right) \subseteq \operatorname{supp}\left(s \partial_{s} \eta_{\varepsilon}\right) \subseteq[-2 \varepsilon,-\varepsilon]$. This implies together with (1.8), (1.9), (1.10), and after taking the expectation,

$$
\underset{t \in[0, \delta)}{\operatorname{ess}-\sup } \mathbb{E} \int_{0}^{L} \Gamma_{\varepsilon}(w(t, \cdot)) \mathrm{d} x \leq \mathbb{E} \int_{0}^{L} \Gamma_{\varepsilon}\left(w_{0}\right) \mathrm{d} x+C \varepsilon \delta,
$$

where $C<\infty$ is independent of $\varepsilon, \delta, w$, and $w_{0}$. Since $\Gamma_{\varepsilon_{1}}(s) \leq \Gamma_{\varepsilon_{2}}(s)$ for $\varepsilon_{1} \geq \varepsilon_{2} \geq 0$ and $s \in \mathbb{R}$, we may take the limit as $\varepsilon \searrow 0$ and get by monotone convergence,

$$
\begin{aligned}
\underset{t \in[0, \delta)}{\operatorname{ess-sup}}\left(-\mathbb{E} \int_{\{w<0\}} w(t, x) \mathrm{d} x\right) & =\underset{t \in[0, \delta)}{\operatorname{ess-sup}} \mathbb{E} \int_{0}^{L} \Gamma_{0}(w(t, x)) \mathrm{d} x \\
& \leq \mathbb{E} \int_{0}^{L} \Gamma_{0}\left(w_{0}\right) \mathrm{d} x=-\mathbb{E} \int_{\left\{w_{0}<0\right\}} w_{0} \mathrm{~d} x=0 .
\end{aligned}
$$

This implies $w \geq 0, \mathbb{P}$-almost surely.

\section{REgUlARITY IN TIME AND UNIFORM BOUNDS OF APPROXIMATE SOLUTIONS}

We use the notations and conventions of $\S 1.3$. For $u_{0} \in H^{1}\left(\mathbb{T}_{L}\right)$ such that $u_{0} \geq 0$, we define for every $N \in \mathbb{N}$ solutions $v_{N}: \Omega \times[0, T) \times \mathbb{T}_{L} \rightarrow[0, \infty)$ and $w_{N}: \Omega \times[0, T) \times \mathbb{T}_{L} \rightarrow$ $[0, \infty)$ according to the splitting scheme (D)-(S)-(DS) through Theorem 2.1, Corollary 2.2, and Proposition 3.2. Note that indeed by Theorem 2.1 (a) and Definition 3.1 the limits

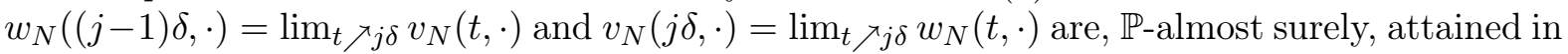


$C^{\frac{1}{2}}\left(\mathbb{T}_{L}\right)$ and $L^{2}\left(\mathbb{T}_{L}\right)$, respectively, and because of (2.3) of Corollary 2.2, (3.6) of Proposition 3.2, and weak lower-semicontunity of the appearing norms, we have $\mathbb{E}\left\|\partial_{x} w_{N}((j-1) \delta, \cdot)\right\|_{2}^{p}<\infty$ and $\left\|\partial_{x} v_{N}(j \delta, \cdot)\right\|_{2}^{p}<\infty$, where $j \in\{1, \ldots, N+1\}$. We further define the concatenated approximate solution $u_{N}: \Omega \times[0, T) \times \mathbb{T}_{L} \rightarrow[0, \infty)$ by

$$
u_{N}(t, \cdot):=\left\{\begin{array}{ll}
v_{N}(2 t-(j-1) \delta, \cdot) & \text { for } t \in\left[(j-1) \delta,\left(j-\frac{1}{2}\right) \delta\right), \\
w_{N}(2 t-j \delta, \cdot) & \text { for } t \in\left[\left(j-\frac{1}{2}\right) \delta, j \delta\right),
\end{array} \quad \text { where } \quad j \in\{1, \ldots, N+1\},\right.
$$

where we recall the notation $\delta=\frac{T}{N+1}$. By Theorem 2.1 and Propositions 2.2 and 3.3 we have $\int_{0}^{L} u_{N} \mathrm{~d} x=\int_{0}^{L} u_{0} \mathrm{~d} x$ in $[0, T), \mathbb{P}$-almost surely, and $u_{N} \geq 0, \mathbb{P}$-almost surely, for every $t \in[0, T)$. Furthermore, we can prove:

Proposition 4.1. For any $p \in[2, \infty)$, there exists a constant $C<\infty$ such that for all $N \in \mathbb{N}$ we have

$$
v_{N}, w_{N} \in L^{p}\left(\Omega, \mathcal{F}, \mathbb{P} ; L^{\infty}\left([0, T) ; H^{1}\left(\mathbb{T}_{L}\right)\right)\right)
$$

with

$$
\begin{aligned}
& \underset{t \in[0, T)}{\mathbb{E} \text { ess-sup }}\left\|u_{N}(t, \cdot)\right\|_{1,2}^{p}+\underset{t \in[0, T)}{\mathbb{E}} \operatorname{ess-sup}_{t \in[}\left\|v_{N}(t, \cdot)\right\|_{1,2}^{p}+\underset{t \in[0, T)}{\mathbb{E} \text { ess-sup }}\left\|w_{N}(t, \cdot)\right\|_{1,2}^{p} \\
& +\mathbb{E} \int_{0}^{T}\left\|v_{N}(t, \cdot)\right\|_{1,2}^{p-2} \int_{\left\{v_{N}(t, \cdot)>0\right\}}\left(v_{N} \partial_{x}^{3} v_{N}\right)^{2} \mathrm{~d} x \mathrm{~d} t \leq C\left\|u_{0}\right\|_{1,2}^{p} .
\end{aligned}
$$

Proof of Proposition 4.1. By Theorem 2.1 (e), Proposition 3.2, and the fact that due to (1.13c) of property (DS) there are no jumps of $u_{N}$ at times $t \in\left\{\frac{\delta}{2}, \ldots,(2 N+1) \frac{\delta}{2}\right\}$, we have

(4.3a) $\int_{0}^{L} u_{0} \mathrm{~d} x=\int_{0}^{L} u_{N} \mathrm{~d} x=\int_{0}^{L} v_{N} \mathrm{~d} x=\int_{0}^{L} w_{N} \mathrm{~d} x \quad$ for all $t \in[0, T), \quad \mathbb{P}$-almost surely,

i.e., the mass is conserved. Since surface energy for the deterministic dynamics (D) is dissipated due to (2.3) of Corollary 2.2 and the growth of the initial value for the stochastic dynamics (S) is controlled due to (3.6b) of Proposition 3.2, we obtain

$$
\begin{aligned}
& \mathbb{E}\left\|\partial_{x} v_{N}(j \delta, \cdot)\right\|_{2}^{p} \leq e^{C_{2} j \delta}\left(\left\|\partial_{x} u_{0}\right\|_{2}^{p}+C_{3} j \delta\left(\int_{0}^{L} u_{0} \mathrm{~d} x\right)^{p}\right), \\
& \mathbb{E}\left\|\partial_{x} w_{N}(j \delta, \cdot)\right\|_{2}^{p} \leq e^{C_{2} j \delta}\left(\left\|\partial_{x} u_{0}\right\|_{2}^{p}+C_{3} j \delta\left(\int_{0}^{L} u_{0} \mathrm{~d} x\right)^{p}\right)
\end{aligned}
$$

for $j \in\{0, \ldots, N\}$, where $C_{2}$ and $C_{3}$ are as in (3.6). The combination of (4.3a) with (4.3b) and (4.3c) utilizing Poincaré's inequalities

$$
c\|\varphi\|_{1,2} \leq\left\|\partial_{x} \varphi\right\|_{2}+\left|\int_{0}^{L} \varphi \mathrm{d} x\right| \leq C\|\varphi\|_{1,2} \quad \text { for fixed } 0<c \leq C<\infty \text { and all } \varphi \in H^{1}\left(\mathbb{T}_{L}\right)
$$

implies that there exists $C<\infty$ such that

$$
\mathbb{E}\left\|v_{N}(j \delta, \cdot)\right\|_{1,2}^{p} \leq C\left\|u_{0}\right\|_{1,2}^{p} \quad \text { and } \quad \mathbb{E}\left\|w_{N}(j \delta, \cdot)\right\|_{1,2}^{p} \leq C\left\|u_{0}\right\|_{1,2}^{p}
$$

for $j \in\{0, \ldots, N\}$. Now combining (4.3) with (2.3) of Corollary 2.2 and (3.6a) of Proposition 3.2 and making use of Poincaré's inequalities once more, we obtain (4.2) upon enlarging $C$.

Proposition 4.2 (regularity in time). For any $p \in[2, \infty), \varepsilon>0, \kappa \in\left(2 \varepsilon, 2 p^{-1}\right) \cap\left(2 \varepsilon, \frac{1}{2}\right]$, and $q \in\left(\frac{2}{\kappa-2 \varepsilon}, \infty\right)$, there exists $C<\infty$ such that for all $N \in \mathbb{N}$ we have

$$
u_{N} \in L^{p}\left(\Omega, \mathcal{F}, \mathbb{P} ; B_{q}^{\frac{\kappa}{2}-\varepsilon, q}\left([0, T) ; B_{q}^{\frac{1}{2}-2 \kappa, q}\left(\mathbb{T}_{L}\right)\right)\right)
$$

with

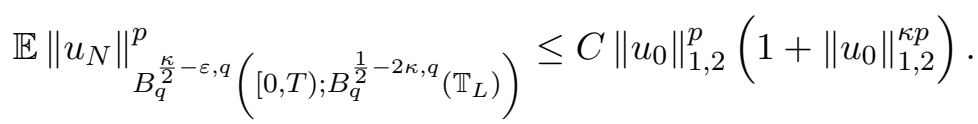


In order to prove Proposition 4.2, we first prove regularity in time for $v_{N}$ and $w_{N}$ separately:

Lemma 4.3. For any $p \in[2, \infty), \varepsilon>0$ and $q \in[p, \infty)$, there exists a constant $C<\infty$ such that for all $N \in \mathbb{N}, j \in\{1, \ldots, N+1\}$, and $\kappa \in\left(0,2 p^{-1}\right)$ we have

$$
v_{N} \in L^{p}\left(\Omega, \mathcal{F}, \mathbb{P} ; B_{q}^{\frac{\kappa}{2}-\varepsilon, q}\left([(j-1) \delta, j \delta) ; B_{q}^{\frac{1}{2}-2 \kappa, q}\left(\mathbb{T}_{L}\right)\right)\right)
$$

with

$$
\mathbb{E}\left(\sum_{j=1}^{N+1}\left\|v_{N}\right\|_{B_{q}^{\frac{\kappa}{2}-\varepsilon, q}}^{q}\left([(j-1) \delta, j \delta) ; B_{q}^{\frac{1}{2}-2 \kappa, q}\left(\mathbb{T}_{L}\right)\right)\right)^{\frac{p}{q}} \leq C\left\|u_{0}\right\|_{1,2}^{p}\left(1+\left\|u_{0}\right\|_{1,2}^{\kappa p}\right) .
$$

Proof of Lemma 4.3. For $(j-1) \delta \leq t_{1} \leq t_{2}<j \delta$ we have from (2.2) of Theorem 2.1 and a localization argument of the appearing test function in time

$$
\left(v_{N}\left(t_{2}, \cdot\right)-v_{N}\left(t_{1}, \cdot\right), \varphi\right)_{2}=\int_{t_{1}}^{t_{2}} \int_{\left\{v_{N}(t, \cdot)>0\right\}} v_{N}^{2}\left(\partial_{x}^{3} v_{N}\right)\left(\partial_{x} \varphi\right) \mathrm{d} x \mathrm{~d} t, \quad \mathbb{P} \text {-almost surely, }
$$

where $\varphi \in C^{\infty}\left(\mathbb{T}_{L}\right)$. Applying the Cauchy-Schwarz inequality leads to

$$
\left\|v_{N}\left(t_{2}, \cdot\right)-v_{N}\left(t_{1}, \cdot\right)\right\|_{H^{-1}\left(\mathbb{T}_{L}\right)} \leq \int_{t_{1}}^{t_{2}}\left(\int_{\left\{v_{N}(t, \cdot)>0\right\}} v_{N}^{4}\left(\partial_{x}^{3} v_{N}\right)^{2} \mathrm{~d} x\right)^{\frac{1}{2}} \mathrm{~d} t, \quad \mathbb{P} \text {-almost surely. }
$$

Hence, we obtain, after using the Sobolev embedding theorem and the Cauchy-Schwarz inequality once more,

$$
\begin{aligned}
\left\|v_{N}\left(t_{2}, \cdot\right)-v_{N}\left(t_{1}, \cdot\right)\right\|_{H^{-1}\left(\mathbb{T}_{L}\right)}^{2} & \leq C\left(\int_{t_{1}}^{t_{2}}\left\|v_{N}(t, \cdot)\right\|_{1,2}\left(\int_{\left\{v_{N}(t, \cdot)>0\right\}} v_{N}^{2}\left(\partial_{x}^{3} v_{N}\right)^{2} \mathrm{~d} x\right)^{\frac{1}{2}} \mathrm{~d} t\right)^{2} \\
& \leq C\left(t_{2}-t_{1}\right) \int_{t_{1}}^{t_{2}}\left\|v_{N}(t, \cdot)\right\|_{1,2}^{2} \int_{\left\{v_{N}(t, \cdot)>0\right\}} v_{N}^{2}\left(\partial_{x}^{3} v_{N}\right)^{2} \mathrm{~d} x \mathrm{~d} t,
\end{aligned}
$$

$\mathbb{P}$-almost surely, so that with help of (4.2) of Proposition 4.1

$$
\left\|v_{N}\right\|_{L^{2}\left(\Omega, \mathcal{F}, \mathbb{P} ; C^{\frac{1}{2}}\left([(j-1) \delta, j \delta) ; H^{-1}\left(\mathbb{T}_{L}\right)\right)\right)} \leq C\left\|u_{0}\right\|_{1,2}^{2},
$$

where $C<\infty$ only depends on $L$. Setting $\tilde{p}:=\frac{2(1-\kappa) p}{2-\kappa p}$ and

$$
\begin{aligned}
& X_{1}:=L^{\infty}\left([(j-1) \delta, j \delta) ; H^{1}\left(\mathbb{T}_{L}\right)\right), \\
& X_{2}:=W^{\frac{1}{2}, \infty}\left([(j-1) \delta, j \delta) ; H^{-1}\left(\mathbb{T}_{L}\right)\right) \hookleftarrow C^{\frac{1}{2}}\left([0, T) ; H^{-1}\left(\mathbb{T}_{L}\right)\right),
\end{aligned}
$$

we infer by interpolation

$$
\begin{array}{cll}
\left\|v_{N}\right\|_{L^{p}\left(\Omega, \mathcal{F}, \mathbb{P} ;\left(X_{1}, X_{2}\right)_{\kappa, p}\right)} & =\left\|v_{N}\right\|_{\left(L^{\tilde{p}}\left(\Omega, \mathcal{F}, \mathbb{P} ; X_{1}\right), L^{2}\left(\Omega, \mathcal{F}, \mathbb{P} ; X_{2}\right)\right)_{\kappa, p}} \\
\leq & C\left\|v_{N}\right\|_{L^{\tilde{p}}\left(\Omega, \mathcal{F}, \mathbb{P} ; X_{1}\right)}^{1-\kappa}\left\|v_{N}\right\|_{L^{2}\left(\Omega, \mathcal{F}, \mathbb{P} ; X_{2}\right)}^{\kappa} \\
& \stackrel{(4.2),(4.6)}{\leq} & C\left\|u_{0}\right\|_{1,2}^{1+\kappa},
\end{array}
$$

where $C<\infty$ only depends on $\kappa$ and $p$, and we have applied [47, Chapitre VII, §1, 1.1, Théorème (1.1)] or equivalently [56, Theorem 1.18.4] in the first line and the standard interpolation inequality in the second line.

Now, we may use $[5$, Theorem 3.4.1 (b)] or [56, §1.3.3, Theorem (d)] and the Sobolev embedding theorem to deduce

provided $p \leq q$, where

$$
\left(X_{1}, X_{2}\right)_{\kappa, p} \hookrightarrow\left(Y_{1}, Y_{2}\right)_{\kappa, q},
$$

$$
Y_{1}:=B_{q}^{-\varepsilon, q}\left([(j-1) \delta, j \delta) ; B_{q}^{\frac{1}{2}, q}\left(\mathbb{T}_{L}\right)\right), \quad Y_{2}:=B_{q}^{\frac{1}{2}, q}\left([(j-1) \delta, j \delta) ; B_{q}^{-\frac{3}{2}, q}\left(\mathbb{T}_{L}\right)\right),
$$


and

$$
\left\|v_{N}\right\|_{\left(Y_{1}, Y_{2}\right)_{\kappa, q}} \leq C \delta^{\frac{1}{q}}\left\|v_{N}\right\|_{\left(X_{1}, X_{2}\right)_{\kappa, p}}
$$

for $C<\infty$ independent of $\delta$ and $j \in\{1, \ldots, N+1\}$. From Lemma B.1 below we infer

with

$$
\left(X_{1}, X_{2}\right)_{\kappa, p} \hookrightarrow B_{q}^{\frac{\kappa}{2}-\varepsilon, q}\left([(j-1) \delta, j \delta) ; B_{q}^{\frac{1}{2}-2 \kappa, q}\left(\mathbb{T}_{L}\right)\right)
$$

$$
\left\|v_{N}\right\|_{B_{q}^{\frac{\kappa}{2}-\varepsilon, q}}\left([(j-1) \delta, j \delta) ; B_{q}^{\frac{1}{2}-2 \kappa, q}\left(\mathbb{T}_{L}\right)\right) \leq C \delta^{\frac{1}{q}}\left\|v_{N}\right\|_{\left(X_{1}, X_{2}\right)_{\kappa, p}},
$$

where $C<\infty$ is independent of $\delta$ and $j \in\{1, \ldots, N+1\}$. In conjunction with (4.7) this implies (4.5) after raising to the power $q$ and summation over $j \in\{1, \ldots, N+1\}$.

Lemma 4.4. For any $p \in[2, \infty), \varepsilon>0$, and $q \in[p, \infty)$, there exists $C<\infty$ such that for all $N \in \mathbb{N}, j \in\{1, \ldots, N+1\}$, and $\gamma \in(0,1)$ we have

$$
w_{N} \in L^{p}\left(\Omega, \mathcal{F}, \mathbb{P} ; B_{q}^{\frac{\gamma}{2}-\varepsilon, q}\left([(j-1) \delta, j \delta) ; B_{q}^{\frac{1}{2}-2 \gamma, q}\left(\mathbb{T}_{L}\right)\right)\right)
$$

with

$$
\mathbb{E}\left(\sum_{j=1}^{N+1}\left\|w_{N}\right\|_{B_{q}^{\frac{\gamma}{2}-\varepsilon, q}}^{q}\left([(j-1) \delta, j \delta) ; B_{q}^{\frac{1}{2}-2 \gamma, q}\left(\mathbb{T}_{L}\right)\right)\right)^{\frac{p}{q}} \leq C\left\|u_{0}\right\|_{1,2}^{p} .
$$

Proof of Lemma 4.4. We derive higher regularity in time $t$ for $w_{N}$. From (3.2), (3.3), (3.4), and (3.5) of Definition 3.1, we infer

$$
\begin{aligned}
w_{N}(t, \cdot)= & w_{N}((j-1) \delta, \cdot)+\underbrace{\int_{(j-1) \delta}^{t} \frac{1}{2} \sum_{k \in \mathbb{Z}} \lambda_{k}^{2} \partial_{x}\left(\psi_{k} \partial_{x}\left(\psi_{k} w_{N}\left(t^{\prime}, \cdot\right)\right)\right) \mathrm{d} t^{\prime}}_{=: w_{N}^{(1)}(t, \cdot)} \\
& +\underbrace{\int_{(j-1) \delta}^{t} \sum_{k \in \mathbb{Z}} \lambda_{k} \partial_{x}\left(\psi_{k} w_{N}\left(t^{\prime}, \cdot\right)\right) \mathrm{d} \beta^{k}\left(t^{\prime}\right)}_{=: w_{N}^{(2)}(t, \cdot)} \text { for } t \in[(j-1) \delta, j \delta),
\end{aligned}
$$

$\mathbb{P}$-almost surely. We conclude that for $(j-1) \delta \leq t_{1} \leq t_{2}<j \delta$ and $\varphi \in C^{\infty}\left(\mathbb{T}_{L}\right)$ we have

$$
\begin{aligned}
\left\|w_{N}^{(1)}\left(t_{2}, \cdot\right)-w_{N}^{(1)}\left(t_{1}, \cdot\right)\right\|_{H^{-1}\left(\mathbb{T}_{L}\right)}^{p} & \stackrel{(1.8),(1.9)}{\leq} C\left(\sum_{k \in \mathbb{Z}} \lambda_{k}^{2} \int_{t_{1}}^{t_{2}}\left\|w_{N}(t, \cdot)\right\|_{1,2} \mathrm{~d} t\right)^{p} \\
& \stackrel{(1.10)}{\leq} C\left|t_{1}-t_{2}\right|^{p} \underset{t \in[0, T)}{\operatorname{ess}-\sup }\left\|w_{N}(t, \cdot)\right\|_{1,2}^{p},
\end{aligned}
$$

$\mathbb{P}$-almost surely, so that with help of (4.2) of Proposition 4.1

$$
\left\|w_{N}^{(1)}\right\|_{L^{p}\left(\Omega, \mathcal{F}, \mathbb{P} ; C^{1-}\left([(j-1) \delta, j \delta] ; H^{-1}\left(\mathbb{T}_{L}\right)\right)\right)} \leq C\left\|u_{0}\right\|_{1,2} .
$$

From [20, Lemma 2.1] we may further deduce for the stochastic integral

$$
\begin{aligned}
& \left\|w_{N}^{(2)}\right\|_{L^{p}\left(\Omega, \mathcal{F}, \mathbb{P} ; W^{\frac{1}{2}-\varepsilon, q}\left([(j-1) \delta, j \delta) ; L^{2}\left(\mathbb{T}_{L}\right)\right)\right)}^{q} \\
& \leq \quad C \mathbb{E} \int_{(j-1) \delta}^{j \delta}\left(\sum_{k \in \mathbb{Z}} \lambda_{k}^{2}\left\|\partial_{x}\left(\psi_{k} w_{N}(t, \cdot)\right)\right\|_{2}^{2}\right)^{\frac{q}{2}} \mathrm{~d} t \\
& \stackrel{(1.8),(1.9),(1.10)}{\leq} C \delta \mathbb{E} \underset{t \in[(j-1) \delta, j \delta)}{\operatorname{ess}-\sup }\left\|w_{N}(t, \cdot)\right\|_{1,2}^{q} \\
& \stackrel{(4.2)}{\leq} \quad C \delta\left\|u_{0}\right\|_{1,2}^{q} \quad \text { for } \quad \alpha<\frac{1}{2} \text {, }
\end{aligned}
$$


where Proposition 4.1 has been used again and $C<\infty$ is independent of $\delta$ and $j \in\{1, \ldots, N+1\}$. This implies by interpolation with (4.2) using [47, Chapitre VII, §1, 1.1, Théorème (1.1)] or [56, Theorem 1.18.4], and scaling in time,

$$
\left\|w_{N}\right\|_{L^{p}\left(\Omega, \mathcal{F}, \mathbb{P} ;\left(X_{1}, X_{2}\right)_{\gamma, p}\right)} \leq C \delta^{\frac{1}{q}}\left\|u_{0}\right\|_{1,2}
$$

where

$$
\begin{aligned}
X_{1} & :=L^{q}\left([(j-1) \delta, j \delta) ; H^{1}\left(\mathbb{T}_{L}\right)\right) \hookrightarrow B_{q}^{-\varepsilon, q}\left([(j-1) \delta, j \delta) ; B_{q}^{\frac{1}{2}, q}\left(\mathbb{T}_{L}\right)\right), \\
X_{2} & :=W^{1, q}\left([(j-1) \delta, j \delta) ; H^{-1}\left(\mathbb{T}_{L}\right)\right)+W^{\frac{1}{2}-\varepsilon, q}\left([(j-1) \delta, j \delta) ; L^{2}\left(\mathbb{T}_{L}\right)\right) \\
& \hookrightarrow B_{q}^{\frac{1}{2}-\varepsilon, q}\left([(j-1) \delta, j \delta) ; B_{q}^{-\frac{3}{2}, q}\left(\mathbb{T}_{L}\right)\right),
\end{aligned}
$$

with $\gamma \in[0,1]$ and where $C<\infty$ is independent of $\delta$ and $j \in\{1, \ldots, N+1\}$. Using [5, Theorem 3.4.1 (b)] or [56, §1.3.3, Theorem (d)] and Lemma B.1 below, we infer

$$
\left(X_{1}, X_{2}\right)_{\gamma, p} \hookrightarrow B_{q}^{\frac{\gamma}{2}-\varepsilon, q}\left([(j-1) \delta, j \delta) ; B_{q}^{\frac{1}{2}-2 \gamma, q}\left(\mathbb{T}_{L}\right)\right),
$$

uniformly in $\delta$ and $j \in\{1, \ldots, N+1\}$, which leads to (4.8) as in the proof of Lemma 4.3.

Proposition 4.2 follows by applying Lemmata 4.3 and 4.4 :

Proof of Proposition 4.2. We may choose $\kappa=\gamma$ in (4.5) and (4.8) of Lemmata 4.3 and 4.4 and note that by construction the function $u_{N}$ does not jump at times $t \in\left\{\frac{\delta}{2}, \ldots,(2 N+1) \frac{\delta}{2}\right\}$. Since by assumption $\frac{\kappa}{2}-\varepsilon-\frac{1}{q}>0$, we have

$$
u_{N} \in L^{p}\left(\Omega, \mathcal{F}, \mathbb{P} ; B C^{0}\left([0, T) ; B_{q}^{\frac{1}{2}-2 \kappa, q}\left(\mathbb{T}_{L}\right)\right)\right)
$$

so that Lemma B.2 below is applicable, giving the bound (4.4).

\section{Convergence of the SPlitting scheme}

In this section, we pass to the limit as $N \rightarrow \infty$ (implying $\delta=\frac{T}{N+1} \rightarrow 0$ ) for the scheme (D)-(S)(DS). We use the notations and conventions introduced in $\S 1.3$ and $\S 4$. Note that the present reasoning is quite similar to the one in $[19, \S 5]$, except for those parts that are specific to the Trotter-Kato scheme (D)-(S)-(DS) and the lack of an interface potential (cf. Proposition 5.6). We also refer to [15, Proposition 5.4] and to [20, Theorem 3.1] for other examples in which analogous arguments have been applied.

5.1. Tightness and convergence of a subsequence. We make use of the following abstract result, which is a generalization of a theorem due to Skorokhod (cf. [55]):

Theorem 5.1 (Jakubowski [38]). Suppose that $(\mathcal{X}, \mathcal{T})$ is a topological space such that there exists a countable family $\left(f_{N}: \mathcal{X} \rightarrow[-1,1]\right)_{N \in \mathbb{N}}$ of $\mathcal{T}$-continuous functions separating points of $\mathcal{X}$. Further assume that $\left(X_{N}\right)_{N \in \mathbb{N}}$ is a sequence of $\mathcal{X}$-valued random variables and that for all $M \in \mathbb{N}$ there exists $\mathcal{K}_{M} \Subset \mathcal{X}$ such that for all $N \in \mathbb{N}$ we have $\mathbb{P}\left\{X_{N} \in \mathcal{K}_{M}\right\}>1-\frac{1}{M}$ (tightness). Then, there exists a subsequence of $\left(X_{N}\right)_{N \in \mathbb{N}}$, denoted by $\left(X_{N}\right)_{N \in \mathbb{N}}$ again, and random variables $\tilde{X}, \tilde{X}_{N}:[0,1] \rightarrow \mathcal{X}$, where $N \in \mathbb{N}$ and $[0,1]$ is equipped with the Borel $\sigma$ algebra, such that $X_{N} \sim \tilde{X}_{N}$ and $\lim _{N \rightarrow \infty} \tilde{X}_{N}(\omega)=\tilde{X}(\omega)$ for all $\omega \in[0,1]$, where the limit is attained in the topology $\mathcal{T}$.

We now apply Theorem 5.1 in order to derive point-wise convergence of in law identical subsequences: 
Proposition 5.2 (point-wise convergence). We define the spaces

$$
\begin{aligned}
\mathcal{X}_{u} & :=B C^{0}\left([0, T) \times \mathbb{T}_{L}\right), \\
\mathcal{X}_{J} & :=L^{2}\left([0, T) \times \mathbb{T}_{L}\right) \quad \text { endowed with the weak topology, } \\
\mathcal{X}_{W} & :=B C^{0}\left([0, T) ; H^{2}\left(\mathbb{T}_{L}\right)\right) .
\end{aligned}
$$

Then, there exist random variables $\tilde{u}, \tilde{u}_{N}:[0,1] \rightarrow \mathcal{X}_{u}, \tilde{J}_{N}, \tilde{J}:[0,1] \rightarrow \mathcal{X}_{J}$, and $\tilde{W}_{N}^{\prime}, \tilde{W}:[0,1] \rightarrow$ $\mathcal{X}_{W}$ with

$$
\left(\tilde{u}_{N}, \tilde{J}_{N}, \tilde{W}_{N}^{\prime}\right) \sim\left(u_{N}, J_{N}, W\right), \quad \text { where } J_{N}:=\mathbb{1}_{\left\{v_{N}>0\right\}} v_{N}^{2}\left(\partial_{x}^{3} v_{N}\right),
$$

as well as $\tilde{u}_{N}(\omega) \rightarrow \tilde{u}(\omega)$ in $\mathcal{X}_{u}, \tilde{J}_{N}(\omega) \rightarrow \tilde{J}(\omega)$ in $\mathcal{X}_{J}$, and $\tilde{W}_{N}^{\prime}(\omega) \rightarrow \tilde{W}(\omega)$ in $\mathcal{X}_{W}$ as $N \rightarrow \infty$, for every $\omega \in[0,1]$, up to taking a subsequence.

Proof of Proposition 5.2. By Markov's inequality, we have for $R>0$ and using Proposition 4.2 with $\varepsilon \in\left(0, \frac{\kappa}{2}\right), p:=2, q \in[2, \infty)$, and $\kappa \in\left[0, \frac{1}{2}\right]$,

$$
\begin{aligned}
\mathbb{P}\left\{\left\|u_{N}\right\|_{B_{q}^{\frac{\kappa}{2}-\varepsilon, q}\left([0, T) ; B_{q}^{\frac{1}{2}-2 \kappa, q}\left(\mathbb{T}_{L}\right)\right)}>R\right\} & \left.\leq \frac{1}{R^{2}} \mathbb{E}\left\|u_{N}\right\|_{B_{q}^{\frac{\kappa}{2}-\varepsilon, q}\left([0, T) ; B_{q}^{\frac{1}{2}-2 \kappa, q}\right.}^{2}\left(\mathbb{T}_{L}\right)\right) \\
& \stackrel{(4.4)}{\leq} \frac{C^{2}}{R^{2}}\left\|u_{0}\right\|_{1,2}^{2}\left(1+\left\|u_{0}\right\|_{1,2}^{2 \kappa}\right) \rightarrow 0 \text { as } R \rightarrow \infty,
\end{aligned}
$$

uniformly in $N \in \mathbb{N}$. Hence,

$$
\mathbb{P}\left\{\left\|u_{N}\right\|_{B_{q}^{\frac{\kappa}{2}-\varepsilon, q}}\left([0, T) ; B_{q}^{\frac{1}{2}-2 \kappa, q}\left(\mathbb{T}_{L}\right)\right) \leq R\right\} \rightarrow 1 \quad \text { as } \quad R \rightarrow \infty,
$$

uniformly in $N \in \mathbb{N}$. Now, for $\kappa<\frac{1}{4}$ and $q>\max \left\{\frac{2}{\kappa-2 \varepsilon}, \frac{2}{1-4 \kappa}\right\}$, by using the compactness result $[1$, Theorem 4.4] and the embedding [52, §3.5.5 Corollary (i)], we infer that

$$
B_{q}^{\frac{1}{2}-2 \kappa, q}\left(\mathbb{T}_{L}\right) \hookrightarrow B C^{0}\left(\mathbb{T}_{L}\right)
$$

is compact because $\frac{1}{2}-2 \kappa-\frac{1}{q}>0$. Once more using [1, Theorem 4.4] and the embeddings $[1,(3.3)$ \& (3.8)], we conclude that

$$
B_{q}^{\frac{\kappa}{2}-\varepsilon, q}\left([0, T) ; B_{q}^{\frac{1}{2}-2 \kappa, q}\left(\mathbb{T}_{L}\right)\right) \hookrightarrow \mathcal{X}_{u}=B C^{0}\left([0, T) \times \mathbb{T}_{L}\right)
$$

is compact because $\frac{\kappa}{2}-\varepsilon-\frac{1}{q}>0$. Therefore, the set

$$
\left\{\|u\|_{B_{q}^{\frac{\kappa}{2}-\varepsilon, q}\left([0, T) ; B_{q}^{\frac{1}{2}-2 \kappa, q}\left(\mathbb{T}_{L}\right)\right)} \leq R\right\}
$$

is a compact subset of $\mathcal{X}_{u}$ for all $R>0$, so that we obtain tightness of $u_{N}$ in $\mathcal{X}_{u}$.

For tightness of $J_{N}$, observe that, again by Markov's inequality and Proposition 4.1,

$$
\begin{aligned}
\mathbb{P}\left\{\left\|J_{N}\right\|_{L^{2}\left([0, T) \times \mathbb{T}_{L}\right)}>R\right\} & \leq \frac{1}{R^{2}} \int_{0}^{T} \mathbb{E} \int_{0}^{L} v_{N}^{4}\left(\partial_{x}^{3} v_{N}\right)^{2} \mathrm{~d} x \mathrm{~d} t \\
& \leq \frac{C}{R^{2}} \mathbb{E} \int_{0}^{T}\left\|v_{N}(t, \cdot)\right\|_{1,2}^{2} \int_{0}^{L} v_{N}^{2}\left(\partial_{x}^{3} v_{N}\right)^{2} \mathrm{~d} x \mathrm{~d} t \\
& \stackrel{(4.2)}{\leq} \frac{C}{R^{2}}\left\|u_{0}\right\|_{1,2}^{4} \rightarrow 0 \text { as } R \rightarrow \infty,
\end{aligned}
$$

uniformly in $N \in \mathbb{N}$, and that $\left\{\|J\|_{L^{2}\left([0, T) \times \mathbb{T}_{L}\right)} \leq R\right\}$ is weakly compact in $L^{2}\left([0, T) \times \mathbb{T}_{L}\right)$. 
For tightness of $W$ in $\mathcal{X}_{W}$ observe that the law of $W, \mu_{W}(\mathcal{A}):=\mathbb{P}\{W \in \mathcal{A}\}$, where $\mathcal{A} \in \mathcal{B}\left(\mathcal{X}_{W}\right)$, is a Radon measure by [40, Theorem 3.16], since $\mathcal{X}_{W}$ is a Polish space. This implies regularity from the interior, i.e.,

$$
1=\mu_{W}\left(\mathcal{X}_{W}\right)=\sup \left\{\mu_{W}(\mathcal{K}): \mathcal{K} \Subset \mathcal{X}_{W}\right\},
$$

which is a reformulation of tightness.

Now the claim follows by application of Theorem 5.1.

In what follows, we assume that the assumptions of Proposition 5.2 are satisfied and we use the notation introduced there. It is convenient to introduce the rescaled and periodically stopped noise

$$
W_{N}(t, \cdot):=\left\{\begin{array}{ll}
W((j-1) \delta, \cdot) & \text { for } \left.t \in\left[(j-1) \delta,\left(j-\frac{1}{2}\right) \delta\right)\right), \\
W(2 t-j \delta, \cdot) & \text { for } t \in\left[\left(j-\frac{1}{2}\right) \delta, j \delta\right),
\end{array} \quad \text { where } \quad j \in\{1, \ldots, N+1\},\right.
$$

$$
\tilde{W}_{N}(t, \cdot):=\left\{\begin{array}{ll}
\tilde{W}_{N}^{\prime}((j-1) \delta, \cdot) & \text { for } \left.t \in\left[(j-1) \delta,\left(j-\frac{1}{2}\right) \delta\right)\right), \\
\tilde{W}_{N}^{\prime}(2 t-j \delta, \cdot) & \text { for } t \in\left[\left(j-\frac{1}{2}\right) \delta, j \delta\right),
\end{array} \text { where } \quad j \in\{1, \ldots, N+1\} .\right.
$$

We define the real-valued processes

$$
\begin{aligned}
\beta_{N}^{k}(t) & :=\lambda_{k}^{-1}\left(W_{N}(t, \cdot), \psi_{k}\right)_{2,2}, \\
\tilde{\beta}_{N}^{k}(t) & :=\lambda_{k}^{-1}\left(\tilde{W}_{N}(t, \cdot), \psi_{k}\right)_{2,2}, \\
\tilde{\beta}^{k}(t) & :=\lambda_{k}^{-1}\left(\tilde{W}(t, \cdot), \psi_{k}\right)_{2,2}
\end{aligned}
$$

so that

$$
W_{N}=\sum_{k \in \mathbb{Z}} \lambda_{k} \psi_{k} \beta_{N}^{k}, \quad \tilde{W}_{N}=\sum_{k \in \mathbb{Z}} \lambda_{k} \psi_{k} \tilde{\beta}_{N}^{k}, \quad \text { and } \quad \tilde{W}=\sum_{k \in \mathbb{Z}} \lambda_{k} \psi_{k} \tilde{\beta}^{k}
$$

Furthermore, we define $\left(\tilde{\mathcal{F}}_{t}\right)_{t \in[0, T)}$ as the augmented filtration of

$$
\tilde{\mathcal{F}}_{t}^{\prime}:=\sigma\left(\tilde{u}\left(t^{\prime}\right), \tilde{J}\left(t^{\prime}\right), \tilde{W}\left(t^{\prime}\right): 0 \leq t^{\prime} \leq t\right)
$$

Proposition 5.3. The processes $\left(\tilde{\beta}^{k}\right)_{k \in \mathbb{Z}}$ are mutually independent standard real-valued $\left(\tilde{\mathcal{F}}_{t}\right)$ Wiener processes.

Proof of Proposition 5.3. We note that $W$ and $\tilde{W}$ as well as $W_{N}$ and $\tilde{W}_{N}$ have the same law and that $W$ and $W_{N}$ take values in $\mathcal{X}_{W}, \mathbb{P}$-almost surely. Hence, also $\tilde{W}$ and $\tilde{W}_{N}$ take values in $\mathcal{X}_{W}, \tilde{\mathbb{P}}$-almost surely. By definitions (5.3b), (5.4b), and (5.4c) this implies that

$$
\tilde{W}_{N} \rightarrow \tilde{W} \quad \text { in } \quad \mathcal{X}_{W} \quad \text { and } \quad \tilde{\beta}_{N}^{k} \rightarrow \tilde{\beta}^{k} \quad \text { in } \quad B C^{0}([0, T)) \quad \text { as } \quad N \rightarrow \infty, \quad \tilde{\mathbb{P}} \text {-almost surely, }
$$

where $k \in \mathbb{Z}$. By definition $(5.4 \mathrm{~b})$, the $\tilde{\beta}^{k}$ are real-valued and $\left(\tilde{\mathcal{F}}_{t}\right)$-adapted. Furthermore, since the joint laws of $\left(\tilde{\beta}^{k}\right)_{k \in \mathbb{Z}}$ and $\left(\beta^{k}\right)_{k \in \mathbb{Z}}$ or $\left(\tilde{\beta}_{N}^{k}\right)_{k \in \mathbb{Z}}$ and $\left(\beta_{N}^{k}\right)_{k \in \mathbb{Z}}$, respectively, coincide, the $\tilde{\beta}^{k}$ or $\tilde{\beta}_{N}^{k}$, respectively, are mutually independent. Then it suffices to show that the $\tilde{\beta}^{k}$ are in fact $\left(\tilde{\mathcal{F}}_{t}\right)$-Wiener processes. This is analogous to [15, Proposition 5.4] or [19, Lemma 5.7], so we only sketch the arguments here.

The first step is to show that

$$
\tilde{\mathbb{E}}\left[\left(\tilde{\beta}^{k}(t)-\tilde{\beta}^{k}\left(t^{\prime}\right)\right) \tilde{\Phi}\right]=0, \quad \text { where } \quad \tilde{\Phi}:=\Phi\left(\left.\tilde{u}\right|_{\left[0, t^{\prime}\right]},\left.\tilde{J}\right|_{\left[0, t^{\prime}\right]},\left.\tilde{W}\right|_{\left[0, t^{\prime}\right]}\right)
$$


and $\Phi \in C^{0}\left(\left.\mathcal{X}_{u}\right|_{\left[0, t^{\prime}\right]} \times\left.\mathcal{X}_{J}\right|_{\left[0, t^{\prime}\right]} \times\left.\mathcal{X}_{W}\right|_{\left[0, t^{\prime}\right]} ;[0,1]\right)$, so that $\tilde{\beta}^{k}$ is an $\left(\tilde{\mathcal{F}}_{t}^{\prime}\right)$-martingale, where again $\tilde{\mathcal{F}}_{t}^{\prime}:=\sigma\left(\tilde{u}\left(t^{\prime \prime}\right), \tilde{J}\left(t^{\prime \prime}\right), \tilde{W}\left(t^{\prime \prime}\right): 0 \leq t^{\prime \prime} \leq t\right)$. This follows from the convergence stated in Proposition 5.2 and (5.5) as well as Vitali's convergence theorem. In the same way, we may conclude that also $\left(\tilde{\beta}^{k}(t)\right)^{2}-t$ is an $\left(\tilde{\mathcal{F}}_{t}^{\prime}\right)$-martingale.

We denote by $\left(\tilde{\mathcal{F}}_{t}^{\prime \prime}\right)_{[0, T)}$ the filtration for which all $\mathbb{P}$-zero sets are added to $\left(\tilde{\mathcal{F}}_{t}^{\prime}\right)_{t \in[0, T)}$. Since $\tilde{\mathcal{F}}_{t^{\prime}}=\bigcap_{t^{\prime \prime}>t^{\prime}} \tilde{\mathcal{F}}_{t^{\prime \prime}}^{\prime \prime}$, continuity in time of $\tilde{\beta}^{k}$ implies with Vitali's convergence theorem

$$
\tilde{\mathbb{E}}\left[\left(\tilde{\beta}^{k}(t)-\tilde{\beta}^{k}\left(t^{\prime}\right)\right) \tilde{\phi}\right]=0
$$

for all $\tilde{\mathcal{F}}_{t^{\prime}}$-measurable and bounded $\tilde{\phi}:[0,1] \rightarrow \mathbb{R}$, so that $\tilde{\beta}^{k}$ is an $\left(\tilde{\mathcal{F}}_{t}\right)$-martingale. The same argument shows that also $\left(\tilde{\beta}^{k}(t)\right)^{2}-t$ is an $\left(\tilde{\mathcal{F}}_{t}\right)$-martingale. By Lévy's characterization theorem (cf. [54, Theorem 3.16]), we infer that the $\tilde{\beta}^{k}$ are $\left(\tilde{\mathcal{F}}_{t}\right)$-Wiener processes.

It is in fact also possible to extract point-wise convergent subsequences of $\tilde{v}_{N}$ and $\tilde{w}_{N}$ (the latter are defined through (4.1), where $u_{N}, v_{N}$, and $w_{N}$ are replaced by $\tilde{u}_{N}, \tilde{v}_{N}$, and $\tilde{w}_{N}$, respectively) and to identify their limits.

Corollary 5.4. Assume that $\tilde{u}_{N}, \tilde{v}_{N}, \tilde{w}_{N}$, and $\tilde{u}$ are given as in Proposition 5.2. Then

$$
\left\|\tilde{u}_{N}-\tilde{u}\right\|_{B C^{0}\left([0, T) \times \mathbb{T}_{L}\right)} \rightarrow 0, \quad\left\|\tilde{v}_{N}-\tilde{u}\right\|_{L^{\infty}\left([0, T) \times \mathbb{T}_{L}\right)} \rightarrow 0, \quad\left\|\tilde{w}_{N}-\tilde{u}\right\|_{L^{\infty}\left([0, T) \times \mathbb{T}_{L}\right)} \rightarrow 0
$$

as $N \rightarrow \infty, \tilde{\mathbb{P}}$-almost surely.

Proof of Corollary 5.4. Since $\mathcal{X}_{u}=B C^{0}\left([0, T) \times \mathbb{T}_{L}\right)$, the first part of (5.6) is a reformulation of Proposition 5.2. In view of (4.1) this implies

$$
\left\|\tilde{v}_{N}-\tilde{u}\right\|_{L^{\infty}\left([0, T) \times \mathbb{T}_{L}\right)} \rightarrow 0 \text { and } \quad\left\|\tilde{w}_{N}-\tilde{u}\right\|_{L^{\infty}\left([0, T) \times \mathbb{T}_{L}\right)} \rightarrow 0 \quad \text { as } \quad N \rightarrow \infty,
$$

$\tilde{\mathbb{P}}$-almost surely. This proves the second and the third limit in (5.6).

Proposition 5.5 (weak convergence, identification of limits, a-priori estimate). Let $\tilde{u}_{N}$ and $\tilde{u}$ be as in Proposition 5.2. Then, there exist subsequences of $\tilde{u}_{N}, \tilde{v}_{N}$ and $\tilde{w}_{N}$, again denoted by $\tilde{u}_{N}, \tilde{v}_{N}$, and $\tilde{w}_{N}$, such that for any $p \in[2, \infty)$,

$$
\tilde{u}_{N} \stackrel{*}{\rightarrow} \tilde{u}, \quad \tilde{v}_{N} \stackrel{*}{\rightarrow} \tilde{u}, \quad \text { and } \quad \tilde{w}_{N} \stackrel{*}{\rightarrow} \tilde{u} \quad \text { as } \quad N \rightarrow \infty \quad \text { in } \quad L^{p}\left([0,1] ; L^{\infty}\left([0, T) ; H^{1}\left(\mathbb{T}_{L}\right)\right)\right)
$$

as $N \rightarrow \infty$. Furthermore,

$$
\underset{t \in[0, T)}{\tilde{\mathbb{E}} \operatorname{ess-sup}}\|\tilde{u}(t, \cdot)\|_{1,2}^{p} \leq C\left\|u_{0}\right\|_{1,2}^{p}
$$

for a constant $C<\infty$ independent of $\tilde{u}$ and $u_{0}$. Hence, $\tilde{u}$ is a bounded continuous $H_{\mathrm{w}}^{1}\left(\mathbb{T}_{L}\right)$-valued process.

Proof of Proposition 5.5. The existence of subsequences meeting (5.7) follows by compactness, employing the bound (4.2) of Proposition 4.1, uniqueness of the limit due to (5.6) of Corollary 5.4, and a diagonal-sequence argument to obtain convergence for all $p \in[2, \infty)$. Because of weak lower-semicontinuity of the norm, estimate (4.2) of Proposition 4.1 translates into (5.8).

Since $\tilde{u} \in L^{\infty}\left([0, T) ; H^{1}\left(\mathbb{T}_{L}\right)\right)$, $\tilde{\mathbb{P}}$-almost surely, any sequence $\left(t_{j}\right) \in[0, T)$ with $t_{j} \rightarrow t \in[0, T)$ as $j \rightarrow \infty$ has a subsequence $\left(t_{j}^{\prime}\right)_{j}$, such that $\tilde{u}\left(t_{j}^{\prime}, \cdot\right)$ weak-*-converges in $H^{1}\left(\mathbb{T}_{L}\right)$, $\tilde{\mathbb{P}}$-almost surely. Since $\tilde{u} \in B C^{0}\left([0, T) \times \mathbb{T}_{L}\right), \tilde{\mathbb{P}}$-almost surely, the limit is uniquely given by $\tilde{u}(t, \cdot)$ and thus also $\tilde{u}\left(t_{j}, \cdot\right) \stackrel{*}{\rightarrow} \tilde{u}(t, \cdot)$ in $H^{1}\left(\mathbb{T}_{L}\right), \tilde{\mathbb{P}}$-almost surely, proving the continuity statement.

We can also identify the flux density: 
Proposition 5.6. Let $\tilde{u}_{N}, \tilde{u}, \tilde{J}_{N}$, and $\tilde{J}$ be as in Proposition 5.2. Then the distributional derivative $\partial_{x}^{3} \tilde{u}$ meets $\partial_{x}^{3} \tilde{u} \in L^{2}(\{\tilde{u}>r\})$ for any $r>0$ and further $\tilde{J}_{N}=\mathbb{1}_{\left\{\tilde{v}_{N}>0\right\}} \tilde{v}_{N}^{2}\left(\partial_{x}^{3} \tilde{v}_{N}\right)$ and $\tilde{J}=\mathbb{1}_{\{\tilde{u}>0\}} \tilde{u}^{2}\left(\partial_{x}^{3} \tilde{u}\right), \tilde{\mathbb{P}}$-almost surely.

Proof of Proposition 5.6. Since by (5.2) of Proposition 5.2 the joint laws coincide, we have for any $\phi \in C^{\infty}\left([0, T] \times \mathbb{T}_{L}\right)$

$$
\begin{aligned}
0 & =\mathbb{E}\left|\int_{0}^{T} \int_{0}^{L} J_{N} \phi \mathrm{d} x \mathrm{~d} t-\int_{0}^{T} \int_{0}^{L} \mathbb{1}_{\left\{v_{N}(t, \cdot)>0\right\}} v_{N}^{2}\left(\partial_{x}^{3} v_{N}\right) \phi \mathrm{d} x \mathrm{~d} t\right| \\
& =\tilde{\mathbb{E}}\left|\int_{0}^{T} \int_{0}^{L} \tilde{J}_{N} \phi \mathrm{d} x \mathrm{~d} t-\int_{0}^{T} \int_{0}^{L} \mathbb{1}_{\left\{\tilde{v}_{N}(t,)>0\right\}} \tilde{v}_{N}^{2}\left(\partial_{x}^{3} \tilde{v}_{N}\right) \phi \mathrm{d} x \mathrm{~d} t\right|,
\end{aligned}
$$

so that indeed $\tilde{J}_{N}=\mathbb{1}_{\left\{\tilde{v}_{N}>0\right\}} \tilde{v}_{N}^{2}\left(\partial_{x}^{3} \tilde{v}_{N}\right)$.

Because of the a-priori estimate (4.2) of Proposition 4.1, we have

$$
\tilde{\mathbb{E}} \int_{0}^{T} \int_{\left\{\tilde{v}_{N}(t, \cdot)>0\right\}} \tilde{v}_{N}^{2}\left(\partial_{x}^{3} \tilde{v}_{N}\right)^{2} \mathrm{~d} x \mathrm{~d} t \leq C\left\|u_{0}\right\|_{1,2}^{2},
$$

where $C<\infty$ only depends on $T$. Hence, for fixed $r>0$ we obtain

$$
\begin{aligned}
& \left.\tilde{\mathbb{E}} \int_{0}^{T} \int_{0}^{L}\left(\partial_{x}^{3} \tilde{v}_{N}\right)^{2} \mathbb{1}_{\left\{\left\|\tilde{v}_{N}-\tilde{u}\right\|_{L}\left([0, T) \times \mathbb{T}_{L}\right)\right.}<\frac{r}{2}\right\} \cap\{\tilde{u}>r\} \\
&
\end{aligned}
$$

so that upon taking a subsequence we obtain by compactness

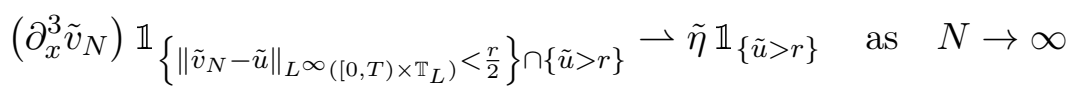

in $L^{2}\left([0,1] \times[0, T) \times \mathbb{T}_{L}\right)$. Taking the limit as $r \searrow 0$, a diagonal-sequence argument implies that, up to taking another subsequence, (5.9) holds true for any $r>0$. Now, for $\tilde{\zeta} \in L^{2}\left([0,1] ; C^{\infty}\left([0, T] \times \mathbb{T}_{L}\right)\right)$ with $\operatorname{supp}_{(t, x) \in[0, T) \times \mathbb{T}_{L}} \tilde{\zeta} \Subset\{\tilde{u}>r\}$ for all $\omega \in[0,1]$, we have

$$
\begin{aligned}
\tilde{\mathbb{E}} \int_{0}^{T} \int_{\{\tilde{u}(t, \cdot)>r\}} \tilde{\eta} \tilde{\zeta} \mathrm{d} x \mathrm{~d} t & \leftarrow \tilde{\mathbb{E}} \int_{0}^{T} \int_{0}^{L}\left(\partial_{x}^{3} \tilde{v}_{N}\right) \mathbb{1}_{\left\{\left\|\tilde{v}_{N}-\tilde{u}\right\|_{L \infty\left([0, T) \times \mathbb{T}_{L}\right)}<\frac{r}{2}\right\} \cap\{\tilde{u}>r\}} \tilde{\zeta} \mathrm{d} x \mathrm{~d} t \\
& =-\tilde{\mathbb{E}} \int_{0}^{T} \int_{0}^{L} \tilde{v}_{N} \mathbb{1}_{\left\{\left\|\tilde{v}_{N}-\tilde{u}\right\|_{L^{\infty}\left([0, T) \times \mathbb{T}_{L}\right)}<\frac{r}{2}\right\} \cap\{\tilde{u}>r\}}\left(\partial_{x}^{3} \tilde{\zeta}\right) \mathrm{d} x \mathrm{~d} t \\
& \rightarrow-\tilde{\mathbb{E}} \int_{0}^{T} \int_{\{\tilde{u}(t, \cdot)>r\}} \tilde{u}\left(\partial_{x}^{3} \tilde{\zeta}\right) \mathrm{d} x \mathrm{~d} t
\end{aligned}
$$

as $N \rightarrow \infty$ for any $r>0$ by using Vitali's convergence theorem in the last line. Application of the latter relies on (5.6) of Corollary 5.4 and

$$
\begin{aligned}
\tilde{\mathbb{E}} \int_{0}^{T} \int_{0}^{L}\left|\tilde{v}_{N}\right|^{\frac{3}{2}}\left|\partial_{x}^{3} \tilde{\zeta}\right|^{\frac{3}{2}} \mathrm{~d} x \mathrm{~d} t & \leq\left(\tilde{\mathbb{E}} \int_{0}^{T} \int_{0}^{L}\left|\tilde{v}_{N}\right|^{6} \mathrm{~d} x \mathrm{~d} t\right)^{\frac{1}{4}}\left(\tilde{\mathbb{E}} \int_{0}^{T} \int_{0}^{L}\left(\partial_{x}^{3} \tilde{\zeta}\right)^{2} \mathrm{~d} x \mathrm{~d} t\right)^{\frac{3}{4}} \\
& \leq C T^{\frac{1}{4}}\left(\underset{\mathbb{E}}{\tilde{\mathbb{E}}_{t \in[0, T)}}\left\|\tilde{v}_{N}(t, \cdot)\right\|_{1,2}^{6}\right)^{\frac{1}{4}} \stackrel{(4.2)}{\leq} C\left\|u_{0}\right\|_{1,2}^{\frac{3}{2}},
\end{aligned}
$$

where $C<\infty$ is independent of $N$ and Hölder's inequality and Proposition 4.1 have been used. Hence, we obtain $\tilde{\eta}=\partial_{x}^{3} \tilde{u}$ distributionally on $\{\tilde{u}>0\}$. For $\tilde{\phi} \in L^{\infty}\left([0,1] \times[0, T] \times \mathbb{T}_{L}\right)$ and $N$ 
sufficiently large, we may split up according to

$$
\begin{aligned}
\tilde{\mathbb{E}} \int_{0}^{T} \int_{\left\{\tilde{v}_{N}(t, \cdot)>0\right\}} \tilde{v}_{N}^{2}\left(\partial_{x}^{3} \tilde{v}_{N}\right) \tilde{\phi} \mathrm{d} x \mathrm{~d} t \\
=\quad \tilde{\mathbb{E}} \int_{0}^{T} \int_{0}^{L} \tilde{v}_{N}^{2}\left(\partial_{x}^{3} \tilde{v}_{N}\right) \mathbb{1}_{\left\{\left\|\tilde{v}_{N}-\tilde{u}\right\|_{L \infty\left([0, T) \times \mathbb{T}_{L}\right)}<\frac{r}{2}\right\} \cap\{\tilde{u}>r\}} \tilde{\phi} \mathrm{d} x \mathrm{~d} t \\
\quad+\tilde{\mathbb{E}} \int_{0}^{T} \int_{\left\{\tilde{v}_{N}(t, \cdot)>0\right\}} \tilde{v}_{N}^{2}\left(\partial_{x}^{3} \tilde{v}_{N}\right) \mathbb{1}_{\left\{\left\|\tilde{v}_{N}-\tilde{u}\right\|_{L_{(}\left([0, T) \times \mathbb{T}_{L}\right)} \geq \frac{r}{2}\right\} \cup\{\tilde{u} \leq r\}} \tilde{\phi} \mathrm{d} x \mathrm{~d} t .
\end{aligned}
$$

Since by Proposition 4.1 and Sobolev embedding

$$
\tilde{\mathbb{E}} \int_{0}^{T} \int_{0}^{L}\left(\tilde{v}_{N}-\tilde{u}\right)^{6} \mathrm{~d} x \mathrm{~d} t \leq C\left(\underset{\mathbb{E}}{\tilde{\mathbb{E}} \operatorname{ess}_{t \in[0, T)} \sup }\left\|\tilde{v}_{N}(t, \cdot)\right\|_{1,2}^{6}+\underset{t \in[0, T)}{\tilde{\mathbb{E}}} \operatorname{ess-sup}_{t \in \tilde{u}}(t, \cdot) \|_{1,2}^{6}\right) \stackrel{(4.2)}{\leq} C\left\|u_{0}\right\|_{1,2}^{6}
$$

and $\left\|\tilde{v}_{N}-\tilde{u}\right\|_{L^{\infty}\left([0, T) \times \mathbb{T}_{L}\right)} \rightarrow 0$ as $N \rightarrow \infty, \tilde{\mathbb{P}}$-almost surely, by (5.6) of Corollary 5.4, it follows by Vitali's convergence theorem that

$$
\tilde{\mathbb{E}} \int_{0}^{T} \int_{0}^{L}\left(\tilde{v}_{N}-\tilde{u}\right)^{4} \mathrm{~d} x \mathrm{~d} t \rightarrow 0 \quad \text { as } \quad N \rightarrow \infty
$$

Hence, we obtain

$$
\begin{aligned}
\tilde{\mathbb{E}} \int_{0}^{T} \int_{0}^{L} \tilde{v}_{N}^{2}\left(\partial_{x}^{3} \tilde{v}_{N}\right) \mathbb{1}_{\left\{\left\|\tilde{v}_{N}-\tilde{u}\right\|_{L^{\infty}\left([0, T) \times \mathbb{T}_{L}\right)}<\frac{r}{2}\right\} \cap\{\tilde{u}>r\}} \tilde{\phi} \mathrm{d} x \mathrm{~d} t \\
\quad \rightarrow \quad \tilde{\mathbb{E}} \int_{0}^{T} \int_{\{\tilde{u}(t, \cdot)>r\}} \tilde{u}^{2}\left(\partial_{x}^{3} \tilde{u}\right) \tilde{\phi} \mathrm{d} x \mathrm{~d} t \quad \text { as } \quad N \rightarrow \infty
\end{aligned}
$$

because of (5.9) and (5.11). Furthermore,

$$
\begin{aligned}
\mid \tilde{\mathbb{E}} \int_{0}^{T} & \int_{\left\{\tilde{v}_{N}(t, \cdot)>0\right\}} \tilde{v}_{N}^{2}\left(\partial_{x}^{3} \tilde{v}_{N}\right) \mathbb{1}_{\left\{\left\|\tilde{v}_{N}-\tilde{u}\right\|_{L^{\infty}\left([0, T) \times \mathbb{T}_{L}\right)} \geq \frac{r}{2}\right\} \cup\{\tilde{u} \leq r\}} \tilde{\phi} \mathrm{d} x \mathrm{~d} t \mid \\
\leq & C\|\tilde{\phi}\|_{L^{\infty}\left([0,1] \times[0, T) \times \mathbb{T}_{L}\right)} \\
& \times\left(\tilde{\mathbb{E}} \int_{0}^{T} \int_{\{\tilde{u}(t, \cdot) \leq r\} \cap\left\{\tilde{v}_{N}(t, \cdot)>0\right\}} \tilde{v}_{N}^{2}\left(\partial_{x}^{3} \tilde{v}_{N}\right)^{2} \mathrm{~d} x \mathrm{~d} t\right)^{\frac{1}{2}} \\
& \times\left(\tilde{\mathbb{E}} \int_{0}^{T} \int_{\left\{\tilde{v}_{N}(t, \cdot)>0\right\}}\left(\tilde{v}_{N}\right)^{2} \mathbb{1}_{\left\{\left\|\tilde{v}_{N}-\tilde{u}\right\|_{L}\left([0, T) \times \mathbb{T}_{L}\right)\right.} \geq \frac{r}{2}\right\} \cup\{\tilde{u} \leq r\} \\
\stackrel{(4.2)}{\leq} & \left.C\|\tilde{\phi}\|_{L^{\infty}\left([0,1] \times[0, T) \times \mathbb{T}_{L}\right)}\left\|u_{0}\right\|_{1,2} \mathrm{~d} t\right)^{\frac{1}{2}} \\
& \times\left(\tilde{\mathbb{E}} \int_{0}^{T} \int_{\left\{\tilde{v}_{N}(t, \cdot)>0\right\}}\left(\tilde{v}_{N}\right)^{2} \mathbb{1}_{\left\{\left\|\tilde{v}_{N}-\tilde{u}\right\|_{L}\left([0, T) \times \mathbb{T}_{L}\right) \geq \frac{r}{2}\right\} \cup\{\tilde{u} \leq r\}} \mathrm{d} x \mathrm{~d} t\right)^{\frac{1}{2}},
\end{aligned}
$$

where $C<\infty$ is independent of $N$ and Proposition 4.1 has been applied. Now, we note that by Sobolev embedding

$$
\begin{aligned}
& \tilde{\mathbb{E}} \int_{0}^{T} \int_{\left\{\tilde{v}_{N}(t, \cdot)>0\right\}}\left(\tilde{v}_{N}\right)^{4} \mathbb{1}_{\left\{\left\|\tilde{v}_{N}-\tilde{u}\right\|_{L^{\infty}\left([0, T) \times \mathbb{T}_{L}\right)} \geq \frac{r}{2}\right\} \cup\{\tilde{u} \leq r\}} \mathrm{d} x \mathrm{~d} t \\
& \leq \quad C \underset{t \in[0, T)}{\operatorname{\mathbb {E}}} \operatorname{ess-sup}_{t \in \tilde{v}_{N}(t, \cdot)\left\|_{1,2}^{4} \stackrel{(4.2)}{\leq} C\right\| u_{0} \|_{1,2}^{4},}
\end{aligned}
$$


where $C<\infty$ is independent of $N$ and, so that by (5.6) of Corollary 5.4 we have by Vitali's convergence theorem

$$
\begin{aligned}
& \tilde{\mathbb{E}} \int_{0}^{T} \int_{\left\{\tilde{v}_{N}(t, \cdot)>0\right\}}\left(\tilde{v}_{N}\right)^{2} \mathbb{1}_{\left\{\left\|\tilde{v}_{N}-\tilde{u}\right\|_{L^{\infty}\left([0, T) \times \mathbb{T}_{L}\right)} \geq \frac{r}{2}\right\} \cup\{\tilde{u} \leq r\}} \mathrm{d} x \mathrm{~d} t \\
& \quad \rightarrow \tilde{\mathbb{E}} \int_{0}^{T} \int_{0}^{L} \tilde{u}^{2} \mathbb{1}_{\{\tilde{u} \leq r\}} \mathrm{d} x \mathrm{~d} t=O\left(r^{2}\right) \quad \text { as } \quad N \rightarrow \infty
\end{aligned}
$$

and (5.13) implies

$$
\tilde{\mathbb{E}} \int_{0}^{T} \int_{\left\{\tilde{v}_{N}(t, \cdot)>0\right\}} \tilde{v}_{N}^{2}\left(\partial_{x}^{3} \tilde{v}_{N}\right) \mathbb{1}_{\left\{\left\|\tilde{v}_{N}-\tilde{u}\right\|_{L^{\infty}\left([0, T) \times \mathbb{T}_{L}\right)} \geq \frac{r}{2}\right\} \cup\{\tilde{u} \leq r\}} \tilde{\phi} \mathrm{d} x \mathrm{~d} t=O(r) \quad \text { as } \quad N \rightarrow \infty .
$$

The limits (5.12) and (5.14) in (5.10) lead to

$$
\begin{aligned}
\tilde{\mathbb{E}} & \int_{0}^{T} \int_{\left\{\tilde{v}_{N}(t, \cdot)>0\right\}} \tilde{v}_{N}^{2}\left(\partial_{x}^{3} \tilde{v}_{N}\right) \tilde{\phi} \mathrm{d} x \mathrm{~d} t \\
& =\tilde{\mathbb{E}} \int_{0}^{T} \int_{\{\tilde{u}(t, \cdot)>r\}} \tilde{u}^{2}\left(\partial_{x}^{3} \tilde{u}\right) \tilde{\phi} \mathrm{d} x \mathrm{~d} t+O(r) \text { as } N \rightarrow \infty \\
& \rightarrow \tilde{\mathbb{E}} \int_{0}^{T} \int_{\{\tilde{u}(t, \cdot)>0\}} \tilde{u}^{2}\left(\partial_{x}^{3} \tilde{u}\right) \tilde{\phi} \mathrm{d} x \mathrm{~d} t \quad \text { as } \quad r \searrow 0 .
\end{aligned}
$$

The last step follows by dominated convergence, where we have employed that the integrand is absolutely integrable. The latter follows from

$$
\tilde{u}^{2}\left|\partial_{x}^{3} \tilde{u}\right||\tilde{\phi}| \leq \tilde{u}^{2}\left|\partial_{x}^{3} \tilde{u}\right|\|\tilde{\phi}\|_{L^{\infty}\left([0,1] \times[0, T) \times \mathbb{T}_{L}\right)}
$$

and the fact that by monotone convergence, the first two lines of (5.15), and the Sobolev embedding theorem,

$$
\begin{aligned}
& \tilde{\mathbb{E}} \int_{0}^{T} \int_{\{\tilde{u}(t, \cdot)>0\}} \tilde{u}^{2}\left|\partial_{x}^{3} \tilde{u}\right| \mathrm{d} x \mathrm{~d} t \\
& =\lim _{r \searrow 0} \tilde{\mathbb{E}} \int_{0}^{T} \int_{\{\tilde{u}(t, \cdot)>r\}} \tilde{u}^{2}\left(\partial_{x}^{3} \tilde{u}\right)\left(\mathbb{1}_{\left\{\partial_{x}^{3} \tilde{u}>0\right\}}-\mathbb{1}_{\left\{\partial_{x}^{3} \tilde{u}<0\right\}}\right) \mathrm{d} x \mathrm{~d} t \\
& =\lim _{N \rightarrow \infty} \tilde{\mathbb{E}} \int_{0}^{T} \int_{\left\{\tilde{v}_{N}(t, \cdot)>0\right\}} \tilde{v}_{N}^{2}\left(\partial_{x}^{3} \tilde{v}_{N}\right)\left(\mathbb{1}_{\left\{\partial_{x}^{3} \tilde{u}>0\right\}}-\mathbb{1}_{\left\{\partial_{x}^{3} \tilde{u}<0\right\}}\right) \mathrm{d} x \mathrm{~d} t \\
& \leq C \lim _{N \rightarrow \infty}\left(\tilde{\mathbb{E}} \int_{0}^{T}\left\|\tilde{v}_{N}(t, \cdot)\right\|_{1,2}^{2} \int_{\left\{\tilde{v}_{N}(t, \cdot)>0\right\}} \tilde{v}_{N}^{2}\left(\partial_{x}^{3} \tilde{v}_{N}\right)^{2} \mathrm{~d} x \mathrm{~d} t\right)^{\frac{1}{2}} \stackrel{(4.2)}{\leq} C\left\|u_{0}\right\|_{1,2}^{2},
\end{aligned}
$$

where $C<\infty$ and Proposition 4.1 was used in the last step.

From (5.15) it follows that

$$
\tilde{J}_{N}=\mathbb{1}_{\left\{\tilde{v}_{N}>0\right\}} \tilde{v}_{N}^{2}\left(\partial_{x}^{3} \tilde{v}_{N}\right) \rightarrow \mathbb{1}_{\{\tilde{u}>0\}} \tilde{u}^{2}\left(\partial_{x}^{3} \tilde{u}\right) \quad \text { in } L^{1}\left([0,1] \times[0, T) \times \mathbb{T}_{L}\right) \text { as } N \rightarrow \infty
$$

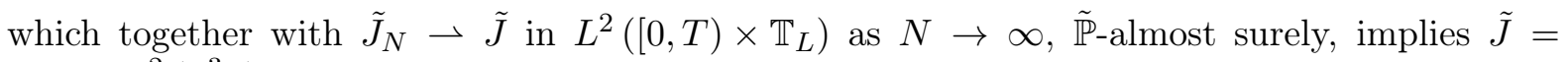
$\mathbb{1}_{\{\tilde{u}>0\}} \tilde{u}^{2}\left(\partial_{x}^{3} \tilde{u}\right)$. 
5.2. Recovering the SPDE. From the scheme (D)-(S)-(DS) we deduce for $t \in[0, T)$ and recalling $\delta=\frac{T}{N+1}$

$$
\begin{aligned}
\left(v_{N}(t, \cdot), \varphi\right)_{2}- & \left(u_{0}, \varphi\right)_{2} \\
\stackrel{(1.13 \mathrm{c})}{=} \quad & \left(v_{N}(t, \cdot), \varphi\right)_{2}+\sum_{j=1}^{\left\lfloor\frac{t}{\delta}\right\rfloor}\left(-\left(v_{N}(j \delta, \cdot), \varphi\right)_{2}+\lim _{t^{\prime} \nearrow j \delta}\left(w_{N}\left(t^{\prime}, \cdot\right), \varphi\right)_{2}\right) \\
& +\sum_{j=1}^{\left\lfloor\frac{t}{\delta}\right\rfloor}\left(\lim _{t^{\prime} \nearrow j \delta}\left(v_{N}\left(t^{\prime}, \cdot\right), \varphi\right)_{2}-\left(w_{N}((j-1) \delta, \cdot), \varphi\right)_{2}\right)-\left(v_{N}(0, \cdot), \varphi\right)_{2} \\
= & \left(v_{N}(t, \cdot), \varphi\right)_{2}-\left(v_{N}\left(\left\lfloor\frac{t}{\delta}\right\rfloor \delta, \cdot\right), \varphi\right)_{2} \\
& +\sum_{j=1}^{\left\lfloor\frac{t}{\delta}\right\rfloor}\left(\lim _{t^{\prime} \nearrow j \delta}\left(v_{N}\left(t^{\prime}, \cdot\right), \varphi\right)_{2}-\left(v_{N}((j-1) \delta, \cdot), \varphi\right)_{2}\right) \\
& +\sum_{j=1}^{\left\lfloor\frac{t}{\delta}\right\rfloor}\left(\lim _{t^{\prime} \nearrow j \delta}\left(w_{N}\left(t^{\prime}, \cdot\right), \varphi\right)_{2}-\left(w_{N}((j-1) \delta, \cdot), \varphi\right)_{2}\right) \\
& \int_{0}^{t} \int_{\left\{v_{N}\left(t^{\prime}, \cdot\right)>0\right\}} v_{N}^{2}\left(\partial_{x}^{3} v_{N}\right)\left(\partial_{x} \varphi\right) \mathrm{d} x \mathrm{~d} t^{\prime} \\
& -\frac{1}{2} \sum_{k \in \mathbb{Z}} \lambda_{k}^{2} \int_{0}^{\left\lfloor\frac{t}{\delta}\right\rfloor \delta}\left(\psi_{k} \partial_{x}\left(\psi_{k} w_{N}\left(t^{\prime}, \cdot\right)\right), \partial_{x} \varphi\right)_{2} \mathrm{~d} t^{\prime} \\
& -\sum_{k \in \mathbb{Z}} \lambda_{k} \int_{0}^{\left\lfloor\frac{t}{\delta}\right\rfloor \delta}\left(\psi_{k} w_{N}\left(t^{\prime}, \cdot\right), \partial_{x} \varphi\right)_{2} \mathrm{~d} \beta^{k}\left(t^{\prime}\right),
\end{aligned}
$$

$\tilde{\mathbb{P}}$-almost surely, where $\varphi \in C^{\infty}\left(\mathbb{T}_{L}\right)$ is a test function. Note that equations (1.13a) and (1.13b) follow rigorously from (2.2) of Theorem 2.1 and (3.5) of Definition 3.1 tested against $\varphi$. Changing the stochastic basis to

$$
\left([0,1], \tilde{\mathcal{F}},\left(\tilde{\mathcal{F}}_{t}\right)_{t \in[0, T)}, \tilde{\mathbb{P}}\right)
$$

we obtain for the in law equivalent convergent subsequences $\tilde{u}_{N}, \tilde{v}_{N}$, and $\tilde{w}_{N}$ for $t \in[0, T)$ by taking (1.7), (4.1), (5.3), and (5.4) into account,

$$
\begin{aligned}
\left(\tilde{v}_{N}(t, \cdot), \varphi\right)_{2}-\left(u_{0}, \varphi\right)_{2}= & \int_{0}^{t} \int_{\left\{\tilde{v}_{N}\left(t^{\prime}, \cdot\right)>0\right\}} \tilde{v}_{N}^{2}\left(\partial_{x}^{3} \tilde{v}_{N}\right) \partial_{x} \varphi \mathrm{d} x \mathrm{~d} t^{\prime} \\
& -\frac{1}{2} \sum_{k \in \mathbb{Z}} \lambda_{k}^{2} \int_{0}^{\left\lfloor\frac{t}{\delta}\right\rfloor \delta}\left(\psi_{k} \partial_{x}\left(\psi_{k} \tilde{w}_{N}\left(t^{\prime}, \cdot\right)\right), \partial_{x} \varphi\right)_{2} \mathrm{~d} t^{\prime} \\
& -\sum_{k \in \mathbb{Z}} \lambda_{k} \int_{0}^{\left\lfloor\frac{t}{\delta}\right\rfloor \delta}\left(\psi_{k} \tilde{u}_{N}\left(t^{\prime}, \cdot\right), \partial_{x} \varphi\right)_{2} \mathrm{~d} \tilde{\beta}_{N}^{k}\left(t^{\prime}\right) .
\end{aligned}
$$

Passing to the limit as $N \rightarrow \infty$, we obtain the main result, Theorem 1.2, by applying Propositions 5.2, 5.3, and 5.5, and showing that the different terms appearing in (5.16) converge in the sense stated in the next lemma: 
Lemma 5.7. Assume that $\tilde{u}_{N}, \tilde{v}_{N}, \tilde{w}_{N}, \tilde{u}, \tilde{v}$, and $\tilde{w}$ are given as in Proposition 5.2 and 5.5. Then, for any $\varphi \in C^{\infty}\left(\mathbb{T}_{L}\right)$ and $t \in[0, T)$, and up to taking subsequences, we have

$$
\begin{aligned}
\left(\tilde{v}_{N}(t, \cdot), \varphi\right)_{2} & \rightarrow(\tilde{u}(t, \cdot), \varphi)_{2} \\
\int_{0}^{t} \int_{\left\{\tilde{v}_{N}\left(t^{\prime}, \cdot\right)>0\right\}} \tilde{v}_{N}^{2}\left(\partial_{x}^{3} \tilde{v}_{N}\right)\left(\partial_{x} \varphi\right) \mathrm{d} x \mathrm{~d} t^{\prime} & \rightarrow \int_{0}^{t} \int_{\left\{\tilde{u}\left(t^{\prime}, \cdot\right)>0\right\}} \tilde{u}^{2}\left(\partial_{x}^{3} \tilde{u}\right)\left(\partial_{x} \varphi\right) \mathrm{d} x \mathrm{~d} t^{\prime}, \\
\sum_{k \in \mathbb{Z}} \lambda_{k}^{2} \int_{0}^{\left\lfloor\frac{t}{\delta}\right\rfloor \delta}\left(\psi_{k} \partial_{x}\left(\psi_{k} \tilde{w}_{N}\left(t^{\prime}, \cdot\right)\right), \partial_{x} \varphi\right)_{2} \mathrm{~d} t^{\prime} & \rightarrow \sum_{k \in \mathbb{Z}} \lambda_{k}^{2} \int_{0}^{t}\left(\psi_{k} \partial_{x}\left(\psi_{k} \tilde{u}\left(t^{\prime}, \cdot\right)\right), \partial_{x} \varphi\right)_{2} \mathrm{~d} t^{\prime}, \\
\sum_{k \in \mathbb{Z}} \lambda_{k} \int_{0}^{\left\lfloor\frac{t}{\delta}\right\rfloor \delta}\left(\psi_{k} \tilde{u}_{N}\left(t^{\prime}, \cdot\right), \partial_{x} \varphi\right)_{2} \mathrm{~d} \tilde{\beta}_{N}^{k}\left(t^{\prime}\right) & \rightarrow \sum_{k \in \mathbb{Z}} \lambda_{k} \int_{0}^{t}\left(\psi_{k} \tilde{u}\left(t^{\prime}, \cdot\right), \partial_{x} \varphi\right)_{2} \mathrm{~d} \tilde{\beta}^{k}\left(t^{\prime}\right)
\end{aligned}
$$

as $N \rightarrow \infty, \tilde{\mathbb{P}}$-almost surely.

Proof of Lemma 5.7. We prove each limit separately:

Proof of (5.17a). Since by (5.6) of Corollary 5.4 we have $\left\|\tilde{v}_{N}-\tilde{u}\right\|_{L^{\infty}\left([0, T) \times \mathbb{T}_{L}\right)} \rightarrow 0$ as $N \rightarrow \infty$, $\tilde{\mathbb{P}}$-almost surely, and $\tilde{v}_{N}$ is $\tilde{\mathbb{P}}$-almost surely piece-wise continuous in time (cf. Theorem 2.1 (a)), it holds

$$
\left\|\tilde{v}_{N}-\tilde{u}\right\|_{B C^{0}\left([0, T) \times \mathbb{T}_{L}\right)}=\left\|\tilde{v}_{N}-\tilde{u}\right\|_{L^{\infty}\left([0, T) \times \mathbb{T}_{L}\right)} \rightarrow 0 \quad \text { as } \quad N \rightarrow \infty .
$$

Hence, we obtain by bounded convergence that $\left(\tilde{v}_{N}(t, \cdot), \varphi\right)_{2} \rightarrow(\tilde{u}(t, \cdot), \varphi)_{2}$ as $N \rightarrow \infty$ for $t \in[0, T), \tilde{\mathbb{P}}$-almost surely, proving (5.17a).

Proof of (5.17b). The limit (5.17b) immediately follows from the weak convergence of the flux density $\tilde{J}_{N}$ stated in Proposition 5.2, i.e., $\tilde{J}_{N}=\mathbb{1}_{\left\{\tilde{v}_{N}>0\right\}} \tilde{v}_{N}^{2}\left(\partial_{x}^{3} v_{N}\right) \rightarrow \tilde{J}$ in $L^{2}\left([0, T) \times \mathbb{T}_{L}\right)$, $\mathbb{P}$-almost surely, and the identification of the limit $\tilde{J}=\mathbb{1}_{\{\tilde{u}>0\}} \tilde{u}^{2}\left(\partial_{x}^{3} \tilde{u}\right)$ given in Proposition 5.6. Proof of (5.17c). We have by (1.8), (1.9), (1.10), (5.6) of Corollary 5.4, and bounded convergence,

$$
\begin{aligned}
& \sum_{k \in \mathbb{Z}} \lambda_{k}^{2} \int_{0}^{\left\lfloor\frac{t}{\delta}\right\rfloor \delta}\left(\psi_{k} \partial_{x}\left(\psi_{k} \tilde{w}_{N}\left(t^{\prime}, \cdot\right)\right), \partial_{x} \varphi\right)_{2} \mathrm{~d} t^{\prime} \\
& \quad=-\sum_{k \in \mathbb{Z}} \lambda_{k}^{2} \int_{0}^{\left\lfloor\frac{t}{\delta}\right\rfloor \delta}\left(\tilde{w}_{N}\left(t^{\prime}, \cdot\right), \psi_{k} \partial_{x}\left(\psi_{k} \partial_{x} \varphi\right)\right)_{2} \mathrm{~d} t^{\prime} \\
& \quad \rightarrow-\sum_{k \in \mathbb{Z}} \lambda_{k}^{2} \int_{0}^{t}\left(\tilde{u}\left(t^{\prime}, \cdot\right), \psi_{k} \partial_{x}\left(\psi_{k} \partial_{x} \varphi\right)\right)_{2} \mathrm{~d} t^{\prime} \quad \text { as } \quad N \rightarrow \infty, \quad \tilde{\mathbb{P}} \text {-almost surely } \\
& \quad=\sum_{k \in \mathbb{Z}} \lambda_{k}^{2} \int_{0}^{t}\left(\psi_{k} \partial_{x}\left(\psi_{k} \tilde{u}\left(t^{\prime}, \cdot\right)\right), \partial_{x} \varphi\right)_{2} \mathrm{~d} t^{\prime},
\end{aligned}
$$

proving $(5.17 \mathrm{c})$.

Proof of (5.17d). For $\varphi \in C^{\infty}\left(\mathbb{T}_{L}\right)$ and $t \in[0, T)$, we define

$$
\begin{aligned}
M_{N, \varphi}(t) & :=\quad-\sum_{k \in \mathbb{Z}} \lambda_{k} \int_{0}^{\left\lfloor\frac{t}{\delta}\right\rfloor \delta}\left(\psi_{k} \tilde{u}_{N}\left(t^{\prime}, \cdot\right), \partial_{x} \varphi\right)_{2} \mathrm{~d} \tilde{\beta}_{N}^{k}\left(t^{\prime}\right) \\
\stackrel{(5.16)}{=} & \left(\tilde{v}_{N}(t, \cdot), \varphi\right)_{2}-\left(u_{0}, \varphi\right)_{2}-\int_{0}^{t} \int_{\left\{\tilde{v}_{N}\left(t^{\prime}, \cdot\right)>0\right\}} \tilde{v}_{N}^{2}\left(\partial_{x}^{3} \tilde{v}_{N}\right)\left(\partial_{x} \varphi\right) \mathrm{d} x \mathrm{~d} t^{\prime} \\
& +\frac{1}{2} \sum_{k \in \mathbb{Z}} \lambda_{k}^{2} \int_{0}^{\left\lfloor\frac{t}{\delta}\right\rfloor \delta}\left(\psi_{k} \partial_{x}\left(\psi_{k} \tilde{w}_{N}\left(t^{\prime}, \cdot\right)\right), \partial_{x} \varphi\right)_{2} \mathrm{~d} t^{\prime} .
\end{aligned}
$$

Note that $\tilde{u}_{N}$ and $\tilde{\beta}_{N}^{k}$ are adapted to $\tilde{\mathcal{F}}_{N, t}:=\sigma\left(\tilde{u}_{N}\left(t^{\prime}, \cdot\right), \tilde{W}_{N}\left(t^{\prime}, \cdot\right): 0 \leq t^{\prime} \leq t\right)$ (We do not need to include $\tilde{J}_{N}$ in view of Proposition 5.6.). In view of (4.1), (5.3), Proposition 5.2, and (5.4b), 
we obtain for the quadratic variation process

$$
\begin{aligned}
\left\langle\left\langle\tilde{M}_{N, \varphi}\right\rangle\right\rangle_{t} & =\sum_{k \in \mathbb{Z}} \lambda_{k}^{2} \int_{0}^{\left\lfloor\frac{t}{\delta}\right\rfloor \delta}\left(\psi_{k} \tilde{w}_{N}\left(t^{\prime}, \cdot\right), \partial_{x} \varphi\right)_{2}^{2} \mathrm{~d} t^{\prime} \\
& \leq \quad\left\|\partial_{x} \varphi\right\|_{2}^{2}\left(\sum_{k \in \mathbb{Z}} \lambda_{k}^{2}\left\|\psi_{k}\right\|_{L^{\infty}\left(\mathbb{T}_{L}\right)}^{2}\right) \int_{0}^{\left\lfloor\frac{t}{\delta}\right\rfloor \delta}\left\|\tilde{w}_{N}\left(t^{\prime}, \cdot\right)\right\|_{2}^{2} \mathrm{~d} t^{\prime} \\
\stackrel{(1.8),(1.10)}{\leq} & C\left\|\partial_{x} \varphi\right\|_{2}^{2} \int_{0}^{\left\lfloor\frac{t}{\delta}\right\rfloor \delta}\left\|\tilde{w}_{N}\left(t^{\prime}, \cdot\right)\right\|_{2}^{2} \mathrm{~d} t^{\prime}
\end{aligned}
$$

so that

$$
\begin{aligned}
\tilde{\mathbb{E}}\left(\left\langle\left\langle\tilde{M}_{N, \varphi}\right\rangle\right\rangle_{t}\right)^{q} & \leq C t^{q}\left\|\partial_{x} \varphi\right\|_{2}^{2 q} \underset{t^{\prime} \in[0, T)}{\operatorname{ess}-\sup } \mathbb{\mathbb { E }}\left\|\tilde{w}_{N}\left(t^{\prime}, \cdot\right)\right\|_{2}^{2 q} \\
& \stackrel{(4.2)}{\leq} C t^{q}\left\|\partial_{x} \varphi\right\|_{2}^{2 q}\left\|u_{0}\right\|_{1,2}^{2 q} \quad \text { for } \quad q \geq 1,
\end{aligned}
$$

where $C<\infty$ is independent of $N$ and Proposition 4.1 has been applied. Hence, $\tilde{M}_{N, \varphi}$ is a square-integrable martingale with respect to $\left(\tilde{\mathcal{F}}_{N, t}\right)_{t \in[0, T)}$. We know from $(5.17 \mathrm{a})-(5.17 \mathrm{c})$ that, for all $t \in[0, T)$,

$$
\begin{aligned}
\tilde{M}_{N, \varphi}(t) \rightarrow \tilde{M}_{\varphi}(t):= & (\tilde{u}(t, \cdot), \varphi)_{2}-\left(u_{0}, \varphi\right)_{2}-\int_{0}^{t} \int_{\left\{\tilde{u}\left(t^{\prime}, \cdot\right)>0\right\}} \tilde{u}^{2}\left(\partial_{x}^{3} \tilde{u}\right)\left(\partial_{x} \varphi\right) \mathrm{d} x \mathrm{~d} t^{\prime} \\
& +\frac{1}{2} \sum_{k \in \mathbb{Z}} \lambda_{k}^{2} \int_{0}^{t}\left(\psi_{k} \partial_{x}\left(\psi_{k} \tilde{u}\left(t^{\prime}, \cdot\right)\right), \partial_{x} \varphi\right)_{2} \mathrm{~d} t^{\prime} \quad \text { as } \quad N \rightarrow \infty,
\end{aligned}
$$

$\tilde{\mathbb{P}}$-almost surely. Then, it suffices to show that, for all $t \in[0, T)$,

$$
\tilde{M}_{\varphi}(t)=-\sum_{k \in \mathbb{Z}} \lambda_{k} \int_{0}^{t}\left(\psi_{k} \tilde{u}\left(t^{\prime}, \cdot\right), \partial_{x} \varphi\right)_{2} \mathrm{~d} \tilde{\beta}^{k}\left(t^{\prime}\right) .
$$

Since $\tilde{M}_{N, \varphi}$ is a square-integrable $\left(\tilde{\mathcal{F}}_{N, t}\right)$-martingale, we have for $0 \leq t^{\prime} \leq t<T$, and

$$
\Phi \in C^{0}\left(\left.\mathcal{X}_{u}\right|_{\left[0, t^{\prime}\right]} \times\left.\mathcal{X}_{W}\right|_{\left[0, t^{\prime}\right]} ;[0,1]\right)
$$

as in (5.1a) and (5.1c) of Proposition 5.2 (Again, it is not necessary to include $\mathcal{X}_{J}$ because of Proposition 5.6.) the identities

$$
\begin{aligned}
& \tilde{\mathbb{E}}\left[\left(\tilde{M}_{N, \varphi}(t)-\tilde{M}_{N, \varphi}\left(t^{\prime}\right)\right) \tilde{\Phi}_{N}\right]=0, \\
& \tilde{\mathbb{E}}\left[\left(\left(\tilde{M}_{N, \varphi}(t)\right)^{2}-\left(\tilde{M}_{N, \varphi}\left(t^{\prime}\right)\right)^{2}-\sum_{k \in \mathbb{Z}} \lambda_{k}^{2} \int_{\left\lfloor\frac{\left.t^{\prime}\right\rfloor}{\delta}\right\rfloor \delta}^{\left\lfloor\frac{t}{\delta}\right\rfloor \delta}\left(\psi_{k} \tilde{w}_{N}\left(t^{\prime \prime}, \cdot\right), \partial_{x} \varphi\right)_{2}^{2} \mathrm{~d} t^{\prime \prime}\right) \tilde{\Phi}_{N}\right]=0, \\
& \tilde{\mathbb{E}}\left[\left(\tilde{\beta}_{N}^{k}(t) \tilde{M}_{N, \varphi}(t)-\tilde{\beta}_{N}^{k}\left(t^{\prime}\right) \tilde{M}_{N, \varphi}\left(t^{\prime}\right)+\lambda_{k} \int_{\left\lfloor\frac{t^{\prime}}{\delta}\right\rfloor \delta}^{\left\lfloor\frac{t}{\delta}\right\rfloor \delta}\left(\psi_{k} \tilde{w}_{N}\left(t^{\prime \prime}, \cdot\right), \partial_{x} \varphi\right)_{2} \mathrm{~d} t^{\prime \prime}\right) \tilde{\Phi}_{N}\right]=0 \text {, }
\end{aligned}
$$

where

$$
\tilde{\Phi}_{N}:=\Phi\left(\left.\tilde{u}_{N}\right|_{\left[0, t^{\prime}\right]},\left.\tilde{W}_{N}\right|_{\left[0, t^{\prime}\right]}\right) .
$$

We derive below that, in the limit as $N \rightarrow \infty$, we have for $0 \leq t^{\prime} \leq t<T$

$$
\begin{aligned}
& \tilde{\mathbb{E}}\left[\left(\tilde{M}_{\varphi}(t)-\tilde{M}_{\varphi}\left(t^{\prime}\right)\right) \tilde{\Phi}\right]=0, \\
& \tilde{\mathbb{E}}\left[\left(\left(\tilde{M}_{\varphi}(t)\right)^{2}-\left(\tilde{M}_{\varphi}\left(t^{\prime}\right)\right)^{2}-\sum_{k \in \mathbb{Z}} \lambda_{k}^{2} \int_{t^{\prime}}^{t}\left(\psi_{k} \tilde{u}\left(t^{\prime \prime}, \cdot\right), \partial_{x} \varphi\right)_{2}^{2} \mathrm{~d} t^{\prime \prime}\right) \tilde{\Phi}\right]=0, \\
& \tilde{\mathbb{E}}\left[\left(\tilde{\beta}^{k}(t) \tilde{M}_{\varphi}(t)-\tilde{\beta}^{k}\left(t^{\prime}\right) \tilde{M}_{\varphi}\left(t^{\prime}\right)+\lambda_{k} \int_{t^{\prime}}^{t}\left(\psi_{k} \tilde{u}\left(t^{\prime \prime}, \cdot\right), \partial_{x} \varphi\right)_{2} \mathrm{~d} t^{\prime \prime}\right) \tilde{\Phi}\right]=0,
\end{aligned}
$$


where

$$
\tilde{\Phi}=\Phi\left(\left.\tilde{u}\right|_{\left[0, t^{\prime}\right]},\left.\tilde{W}\right|_{\left[0, t^{\prime}\right]}\right)
$$

With the same argumentation as in the proof of Proposition 5.3, we may then infer that $\tilde{M}_{\varphi}$ is also an $\left(\tilde{\mathcal{F}}_{t}\right)$-martingale. Hence, (5.21) follows from (1.8), (1.10), and [35, Proposition A.1] or [46].

In order to prove (5.23), we note that

$$
\left|\tilde{\Phi}_{N}\right| \leq 1 \quad \text { and } \quad \tilde{\Phi}_{N} \rightarrow \tilde{\Phi} \quad \text { as } N \rightarrow \infty \text { point-wise, } \tilde{\mathbb{P}} \text {-almost surely. }
$$

Argument for (5.23a). From (5.18) and (5.22a) we deduce

$$
\begin{aligned}
0= & \tilde{\mathbb{E}}\left[\left(\left(\tilde{v}_{N}(t, \cdot)-\tilde{v}_{N}\left(t^{\prime}, \cdot\right), \varphi\right)_{2}-\int_{t^{\prime}}^{t} \int_{\left\{\tilde{v}_{N}\left(t^{\prime \prime}, \cdot\right)>0\right\}} \tilde{v}_{N}^{2}\left(\partial_{x}^{3} \tilde{v}_{N}\right)\left(\partial_{x} \varphi\right) \mathrm{d} x \mathrm{~d} t^{\prime \prime}\right) \tilde{\Phi}_{N}\right] \\
& +\frac{1}{2} \tilde{\mathbb{E}}\left[\left(\sum_{k \in \mathbb{Z}} \lambda_{k}^{2} \int_{\left\lfloor\frac{t^{\prime}}{\delta}\right\rfloor \delta}^{\left\lfloor\frac{t}{\delta}\right\rfloor \delta}\left(\psi_{k} \partial_{x}\left(\psi_{k} \tilde{w}_{N}\left(t^{\prime \prime}, \cdot\right)\right), \partial_{x} \varphi\right)_{2} \mathrm{~d} t^{\prime \prime}\right) \tilde{\Phi}_{N}\right] .
\end{aligned}
$$

Then, we note that

$$
\tilde{\mathbb{E}}\left[\left(\tilde{v}_{N}(t, \cdot)-\tilde{v}_{N}\left(t^{\prime}, \cdot\right), \varphi\right)_{2} \tilde{\Phi}_{N}\right] \rightarrow \tilde{\mathbb{E}}\left[\left(\tilde{u}(t, \cdot)-\tilde{u}\left(t^{\prime}, \cdot\right), \varphi\right)_{2} \tilde{\Phi}\right] \quad \text { as } \quad N \rightarrow \infty .
$$

Indeed, from (5.6) of Corollary 5.4 and piece-wise continuity in time by (4.1), we infer

$$
\left\|\tilde{v}_{N}(t, \cdot)-\tilde{v}_{N}\left(t^{\prime}, \cdot\right)-\tilde{u}(t, \cdot)+\tilde{u}\left(t^{\prime}, \cdot\right)\right\|_{L^{\infty}\left(\mathbb{T}_{L}\right)} \rightarrow 0 \quad \text { as } \quad N \rightarrow \infty,
$$

$\tilde{\mathbb{P}}$-almost surely,

$$
\tilde{\mathbb{E}}\left[\left|\left(\tilde{v}_{N}(t, \cdot)-\tilde{v}_{N}\left(t^{\prime}, \cdot\right), \varphi\right)_{2}\right|^{2}\left(\tilde{\Phi}_{N}\right)^{2}\right] \stackrel{(5.24)}{\leq} 4 \underset{t^{\prime \prime} \in[0, T)}{\operatorname{ess}} \sup \tilde{\mathbb{E}}\left\|\tilde{v}_{N}\left(t^{\prime \prime}, \cdot\right)\right\|_{2}^{2}\|\varphi\|_{2}^{2} \stackrel{(4.2)}{\leq} C\left\|u_{0}\right\|_{1,2}^{2}\|\varphi\|_{2}^{2},
$$

where $C<\infty$ is independent of $N$ and Proposition 4.1 has been applied, so that with (5.24) the claim (5.25) follows by Vitali's convergence theorem.

We argue again by Vitali's convergence theorem to infer that

$$
\tilde{\mathbb{E}}\left[\int_{t^{\prime}}^{t} \int_{\left\{\tilde{v}_{N}\left(t^{\prime \prime}, \cdot\right)>0\right\}} \tilde{v}_{N}^{2}\left(\partial_{x}^{3} \tilde{v}_{N}\right)\left(\partial_{x} \varphi\right) \mathrm{d} x \mathrm{~d} t^{\prime \prime} \tilde{\Phi}_{N}\right] \rightarrow \tilde{\mathbb{E}}\left[\int_{t^{\prime}}^{t} \int_{\left\{\tilde{u}\left(t^{\prime \prime}, \cdot\right)>0\right\}} \tilde{u}^{2}\left(\partial_{x}^{3} \tilde{u}\right)\left(\partial_{x} \varphi\right) \mathrm{d} x \mathrm{~d} t^{\prime \prime} \tilde{\Phi}\right]
$$

as $N \rightarrow \infty$. Indeed, this follows from (5.17b), (5.24), and

$$
\begin{aligned}
& \tilde{\mathbb{E}}\left[\left|\int_{t^{\prime}}^{t} \int_{\left\{\tilde{v}_{N}\left(t^{\prime \prime}, \cdot\right)>0\right\}} \tilde{v}_{N}^{2}\left(\partial_{x}^{3} \tilde{v}_{N}\right)\left(\partial_{x} \varphi\right) \mathrm{d} x \mathrm{~d} t^{\prime \prime}\right|^{2}\left(\tilde{\Phi}_{N}\right)^{2}\right] \\
& \quad \leq C\left\|\partial_{x} \varphi\right\|_{2}^{2} \tilde{\mathbb{E}} \int_{0}^{T}\left\|\tilde{v}_{N}\left(t^{\prime \prime}, \cdot\right)\right\|_{1,2}^{2} \int_{\left\{\tilde{v}_{N}\left(t^{\prime \prime}, \cdot\right)>0\right\}} \tilde{v}_{N}^{2}\left(\partial_{x}^{3} \tilde{v}_{N}\right)^{2} \mathrm{~d} x \mathrm{~d} t^{\prime \prime} \\
& \stackrel{(4.2)}{\leq} C\left\|\partial_{x} \varphi\right\|_{2}^{2}\left\|u_{0}\right\|_{1,2}^{4},
\end{aligned}
$$

where $C<\infty$ is independent of $N$ and Proposition 4.1 has been applied. 
Finally, using (1.8), (1.9b), (1.10), Proposition 4.1, (5.6) of Corollary 5.4, (5.24), and Vitali's convergence theorem, we have

$$
\begin{aligned}
\tilde{\mathbb{E}} & {\left[\left(\sum_{k \in \mathbb{Z}} \lambda_{k}^{2} \int_{\left\lfloor\frac{t^{\prime}}{\delta}\right\rfloor \delta}^{\left\lfloor\frac{t}{\delta}\right\rfloor \delta}\left(\psi_{k} \partial_{x}\left(\psi_{k} \tilde{w}_{N}\left(t^{\prime}, \cdot\right)\right), \partial_{x} \varphi\right)_{2} \mathrm{~d} t^{\prime \prime}\right) \tilde{\Phi}_{N}\right] } \\
=-\tilde{\mathbb{E}} & {\left[\left(\sum_{k \in \mathbb{Z}} \lambda_{k}^{2} \int_{\left\lfloor\frac{t^{\prime}}{\delta}\right\rfloor \delta}^{\left\lfloor\frac{t}{\delta}\right\rfloor \delta}\left(\tilde{w}_{N}\left(t^{\prime}, \cdot\right), \psi_{k} \partial_{x}\left(\psi_{k} \partial_{x} \varphi\right)\right)_{2} \mathrm{~d} t^{\prime \prime}\right) \tilde{\Phi}_{N}\right] } \\
\rightarrow-\tilde{\mathbb{E}} & {\left[\left(\sum_{k \in \mathbb{Z}} \lambda_{k}^{2} \int_{t^{\prime}}^{t}\left(\tilde{u}\left(t^{\prime}, \cdot\right), \psi_{k} \partial_{x}\left(\psi_{k} \partial_{x} \varphi\right)\right)_{2} \mathrm{~d} t^{\prime \prime}\right) \tilde{\Phi}\right] \text { as } N \rightarrow \infty } \\
= & \mathbb{E}\left[\left(\sum_{k \in \mathbb{Z}} \lambda_{k}^{2} \int_{t^{\prime}}^{t}\left(\psi_{k} \partial_{x}\left(\psi_{k} \tilde{u}\left(t^{\prime}, \cdot\right)\right), \partial_{x} \varphi\right)_{2} \mathrm{~d} t^{\prime \prime}\right) \tilde{\Phi}\right]
\end{aligned}
$$

Altogether, we infer that taking the limit as $N \rightarrow \infty$ in (5.22a), we may conclude that (5.23a) holds true.

Argument for (5.23b). First, we note that

$$
\begin{aligned}
\tilde{\mathbb{E}} & \left(\sum_{k \in \mathbb{Z}} \lambda_{k}^{2} \int_{\left\lfloor\frac{t^{\prime}}{\delta}\right\rfloor \delta}^{\left\lfloor\frac{t}{\delta}\right\rfloor \delta}\left(\psi_{k} \tilde{w}_{N}\left(t^{\prime \prime}, \cdot\right), \partial_{x} \varphi\right)_{2}^{2} \mathrm{~d} t^{\prime \prime}\right)^{2} \\
& \stackrel{(1.8),(1.10)}{\leq} C\left\|\partial_{x} \varphi\right\|_{2}^{4} \operatorname{ess}^{-\sup _{t^{\prime \prime} \in[0, T)}} \tilde{\mathbb{E}}\left\|\tilde{w}_{N}\left(t^{\prime \prime}, \cdot\right)\right\|_{2}^{4} \stackrel{(4.2)}{\leq} C\left\|\partial_{x} \varphi\right\|_{2}^{4}\left\|u_{0}\right\|_{1,2}^{4},
\end{aligned}
$$

where $C<\infty$ is independent of $N$ and Proposition 4.1 has been utilized. Additionally, by (5.6) of Corollary 5.4 and bounded convergence

$$
\sum_{k \in \mathbb{Z}} \lambda_{k}^{2} \int_{\left\lfloor\frac{t^{\prime}}{\delta}\right\rfloor \delta}^{\left\lfloor\frac{t}{\delta}\right\rfloor \delta}\left(\psi_{k} \tilde{w}_{N}\left(t^{\prime \prime}, \cdot\right), \partial_{x} \varphi\right)_{2}^{2} \mathrm{~d} t^{\prime \prime} \rightarrow \sum_{k \in \mathbb{Z}} \lambda_{k}^{2} \int_{t^{\prime}}^{t}\left(\psi_{k} \tilde{u}\left(t^{\prime \prime}, \cdot\right), \partial_{x} \varphi\right)_{2}^{2} \mathrm{~d} t^{\prime \prime} \quad \text { as } N \rightarrow \infty
$$

$\tilde{\mathbb{P}}$-almost surely. Together with (5.24) this implies

$$
\tilde{\mathbb{E}}\left[\sum_{k \in \mathbb{Z}} \lambda_{k}^{2} \int_{\left\lfloor\frac{t^{\prime}}{\delta}\right\rfloor \delta}^{\left\lfloor\frac{t}{\delta}\right\rfloor \delta}\left(\psi_{k} \tilde{w}_{N}\left(t^{\prime}, \cdot\right), \partial_{x} \varphi\right)_{2}^{2} \mathrm{~d} t^{\prime \prime} \tilde{\Phi}_{N}\right] \rightarrow \tilde{\mathbb{E}}\left[\sum_{k \in \mathbb{Z}} \lambda_{k}^{2} \int_{t^{\prime}}^{t}\left(\psi_{k} \tilde{u}\left(t^{\prime \prime}, \cdot\right), \partial_{x} \varphi\right)_{2}^{2} \mathrm{~d} t^{\prime \prime} \tilde{\Phi}\right]
$$

as $N \rightarrow \infty$ by Vitali's convergence theorem. Now, by (5.20),

$$
\tilde{M}_{N, \varphi}(t) \rightarrow \tilde{M}_{\varphi}(t) \quad \text { and } \quad \tilde{M}_{N, \varphi}\left(t^{\prime}\right) \rightarrow \tilde{M}_{\varphi}\left(t^{\prime}\right) \quad \text { as } N \rightarrow \infty, \tilde{\mathbb{P}} \text {-almost surely }
$$

and further applying the Burkholder-Davis-Gundy inequality (cf. [54, Theorem 3.28]) gives

$$
\tilde{\mathbb{E}}\left[\left(\tilde{M}_{N, \varphi}(t)\right)^{4}\left(\tilde{\Phi}_{N}\right)^{2}\right] \stackrel{(5.24)}{\leq} C \tilde{\mathbb{E}}\left\langle\left\langle\tilde{M}_{N, \varphi}\right\rangle\right\rangle_{t}^{2} \stackrel{(5.19)}{\leq} C t^{2}\left\|\partial_{x} \varphi\right\|_{2}^{4}\left\|u_{0}\right\|_{1,2}^{4}
$$

where $C<\infty$ is independent of $N$, so that by Vitali's convergence theorem

$$
\tilde{\mathbb{E}}\left[\left(\tilde{M}_{N, \varphi}(t)\right)^{2} \tilde{\Phi}_{N}\right] \rightarrow \tilde{\mathbb{E}}\left[\left(\tilde{M}_{\varphi}(t)\right)^{2} \tilde{\Phi}\right] \text { and } \quad \tilde{\mathbb{E}}\left[\left(\tilde{M}_{N, \varphi}\left(t^{\prime}\right)\right)^{2} \tilde{\Phi}_{N}\right] \rightarrow \tilde{\mathbb{E}}\left[\left(\tilde{M}_{\varphi}\left(t^{\prime}\right)\right)^{2} \tilde{\Phi}\right]
$$

as $N \rightarrow \infty$, where (5.24) has been used once more. Therefore, (5.23b) follows by taking the limit as $N \rightarrow \infty$ in (5.22b).

Argument for (5.23c). With the same reasoning as before, we have

$$
\tilde{\mathbb{E}}\left[\int_{\left\lfloor\frac{t^{\prime}}{\delta}\right\rfloor \delta}^{\left\lfloor\frac{t}{\delta}\right\rfloor \delta}\left(\psi_{k} \tilde{w}_{N}\left(t^{\prime \prime}, \cdot\right), \partial_{x} \varphi\right)_{2} \mathrm{~d} t^{\prime \prime} \tilde{\Phi}_{N}\right] \rightarrow \tilde{\mathbb{E}}\left[\int_{t^{\prime}}^{t}\left(\psi_{k} \tilde{u}\left(t^{\prime \prime}, \cdot\right), \partial_{x} \varphi\right)_{2} \mathrm{~d} t^{\prime \prime} \tilde{\Phi}\right] \quad \text { as } \quad N \rightarrow \infty .
$$


Furthermore, with help of the Cauchy-Schwarz and the Burkholder-Davis-Gundy inequality (cf. [54, Theorem 3.28])

$$
\begin{aligned}
\tilde{\mathbb{E}}\left[\left(\tilde{\beta}_{N}^{k}(t)\right)^{2}\left(\tilde{M}_{N, \varphi}(t)\right)^{2}\left(\tilde{\Phi}_{N}\right)^{2}\right] & \stackrel{(5.24)}{\leq} \sqrt{\tilde{\mathbb{E}}\left(\tilde{\beta}_{N}^{k}(t)\right)^{4}} \sqrt{\tilde{\mathbb{E}}\left(\tilde{M}_{N, \varphi}(t)\right)^{4}} \\
& \leq C \sqrt{\left.\tilde{\mathbb{E}}_{\langle}\left\langle\tilde{\beta}_{N}^{k}\right\rangle\right\rangle_{t}^{2}} \sqrt{\tilde{\mathbb{E}}\left\langle\left\langle\tilde{M}_{N, \varphi}\right\rangle\right\rangle_{t}^{2}} \\
& \stackrel{(5.19)}{\leq} C t^{2}\left\|\partial_{x} \varphi\right\|_{2}^{2}\left\|u_{0}\right\|_{1,2}^{2},
\end{aligned}
$$

where $C<\infty$ is independent of $N$, which implies with $\tilde{\beta}_{N}^{k} \rightarrow \tilde{\beta}_{N}$ as $N \rightarrow \infty$ uniformly in $[0, T), \tilde{\mathbb{P}}$-almost surely, by Proposition 5.2 and (5.4b), (5.24), and $\tilde{M}_{N, \varphi}(t) \rightarrow \tilde{M}_{\varphi}(t)$ as $N \rightarrow \infty$, $\tilde{\mathbb{P}}$-almost surely, by $(5.20)$, the limits

$$
\begin{aligned}
\tilde{\mathbb{E}}\left[\tilde{\beta}^{k}(t) \tilde{M}_{N, \varphi}(t) \tilde{\Phi}_{N}\right] & \rightarrow \tilde{\mathbb{E}}\left[\tilde{\beta}^{k}(t) \tilde{M}_{\varphi}(t) \tilde{\Phi}\right], \\
\tilde{\mathbb{E}}\left[\tilde{\beta}_{N}^{k}\left(t^{\prime}\right) \tilde{M}_{N, \varphi}\left(t^{\prime}\right) \tilde{\Phi}_{N}\right] & \rightarrow \tilde{\mathbb{E}}\left[\tilde{\beta}^{k}\left(t^{\prime}\right) \tilde{M}_{\varphi}\left(t^{\prime}\right) \tilde{\Phi}\right]
\end{aligned}
$$

as $N \rightarrow \infty$ by Vitali's convergence theorem. Hence, (5.23c) follows from (5.22c).

\section{CONCluding REMARKS}

The Trotter-Kato splitting scheme (D)-(S)-(DS), utilized in the present work for the construction of solutions to (1.1), can also be used for the design of a suitable numerical scheme. Hence, an interesting direction for future research may be to further develop the present analysis to prove the convergence of this or a similar numerical algorithm. A numerical treatment of the stochastic thin-film equation with Itô noise and an additional interface potential has been introduced by Grün, Mecke, and Rauscher in [33, §3.1]. Furthermore, it may be of interest to test whether employing Stratonovich noise leads to different findings in the droplet formation simulations carried out in [33].

It appears to be challenging to investigate the stochastic thin-film equation

$$
\mathrm{d} u=-\partial_{x}\left(u^{n} \partial_{x}^{3} u\right) \mathrm{d} t+\partial_{x}\left(u^{\frac{n}{2}} \circ \mathrm{d} W\right),
$$

where $n \in[1,3]$ and where the cubic mobility $n=3$ (corresponding to no slip at the substrate) is of particular interest. In this case, however, the noise is nonlinear and singular for $n<2$, so that for instance shocks in the stochastic dynamics may form. Hence, we expect the analysis in this situation to be significantly more involved. For relevant analysis in the case of the second-order SPDE

$$
\mathrm{d} u=\Delta u^{m} \mathrm{~d} t+\nabla \cdot\left(u^{p} \circ \mathrm{d} W\right)
$$

we refer to the works $[15,22,23]$.

It should also be noted that, besides the weak solution approach, an extensive theory of classical solutions to the thin-film equation, based on maximal-regularity estimates of the linearized evolution, has been developed, starting with the works of Bringmann, Giacomelli, Knüpfer, and Otto $[9,25,26]$ for linear mobility in one space dimension and with zero contact angle and later on further developed to include nonlinear mobilities, nonzero contact angles, and higher dimensions in $[18,24,28,29,39,41-43]$. On the other hand, there have been recent developments in the theory of mild solutions and maximal regularity for stochastic partial differential equations due to van Neerven, Veraar, and Weis [57,58] and Hornung [36]. It would be a viable goal to combine these techniques in order to obtain a stronger control of the solution.

Finally, it would be an illuminating task to study the self-similar behavior of the stochastic thin-film equation (6.1) analytically and thus to lift the numerical findings and dimensional analysis of Davidovitch, Moro, and Stone in [17] to full mathematical rigor. Note that again analytic results in the deterministic case have been obtained for the thin-film equation with 
linear mobility, starting with the works of Bernoff and Witelski in [7] and Carrillo and Toscani in [12] and later on upgraded in [10,11,27, 50,51,53].

We believe that all questions detailed above are interesting future directions, but appear to be analytically quite challenging to address.

\section{Appendix A. Viscous Regularization of Stochastic Dynamics}

Let $\left(\Omega, \mathcal{F},\left(\mathcal{F}_{t}\right)_{t \in[0, \delta]}, \mathbb{P}\right)$ be a complete filtered probability space with a complete and rightcontinuous filtration $\left(\mathcal{F}_{t}\right)_{t \in[0, \delta]}$. Further write $\left(\beta^{k}\right)_{k \in \mathbb{Z}}$ for mutually independent standard realvalued $\left(\mathcal{F}_{t}\right)$-Wiener processes. Consider the viscous regularization

$$
\mathrm{d} w^{\varepsilon}=\left(\frac{1}{2} \sum_{k \in \mathbb{Z}} \lambda_{k}^{2} \partial_{x}\left(\psi_{k} \partial_{x}\left(\psi_{k} w^{\varepsilon}\right)\right)+\varepsilon \partial_{x}^{2} w^{\varepsilon}\right) \mathrm{d} t+\sum_{k \in \mathbb{Z}} \lambda_{k} \partial_{x}\left(\psi_{k} w^{\varepsilon}\right) \mathrm{d} \beta^{k} \quad \text { on } \quad[0, \delta)
$$

of equation (3.1), where $\varepsilon \in(0,1]$. Our aim is to construct a variational solution to (A.1). Therefore, we introduce the operators

$$
A^{\varepsilon}: H^{2}\left(\mathbb{T}_{L}\right) \rightarrow L^{2}\left(\mathbb{T}_{L}\right), \quad w \mapsto \frac{1}{2} \sum_{k \in \mathbb{Z}} \lambda_{k}^{2} \partial_{x}\left(\psi_{k} \partial_{x}\left(\psi_{k} w\right)\right)+\varepsilon \partial_{x}^{2} w
$$

and the diagonal Hilbert-Schmidt-valued operator

$$
B: H^{2}\left(\mathbb{T}_{L}\right) \rightarrow L_{2}\left(H^{2}\left(\mathbb{T}_{L}\right) ; H^{1}\left(\mathbb{T}_{L}\right)\right), \quad w \mapsto\left(v \mapsto \sum_{k \in \mathbb{Z}} \lambda_{k}\left(v, \psi_{k}\right)_{2,2}\left(\partial_{x}\left(\psi_{k} w\right)\right)\right),
$$

Equation (A.1) then attains the abstract form

$$
\mathrm{d} w^{\varepsilon}=A^{\varepsilon} w^{\varepsilon} \mathrm{d} t+\left(B w^{\varepsilon}\right) \mathrm{d} W_{H^{2}\left(\mathbb{T}_{L}\right)},
$$

where

$$
W_{H^{2}\left(\mathbb{T}_{L}\right)}:=\sum_{k \in \mathbb{Z}} \beta^{k} \psi_{k}
$$

is a cylindrical $\left(\mathcal{F}_{t}\right)$-Wiener process in $H^{2}\left(\mathbb{T}_{L}\right)$. The underlying Gelfand triple is

$$
\left(L^{2}\left(\mathbb{T}_{L}\right), H^{1}\left(\mathbb{T}_{L}\right), H^{2}\left(\mathbb{T}_{L}\right)\right) .
$$

We use the following notion of solutions (see [49, Definition 5.1.2]):

Definition A.1. A variational solution to $(\mathrm{A} .4)$ is a continuous $\left(\mathcal{F}_{t}\right)$-adapted $H^{1}\left(\mathbb{T}_{L}\right)$-valued process $w^{\varepsilon}$ such that

$$
\hat{w}^{\varepsilon} \in L^{2}\left([0, \delta) \times \Omega, \mathrm{d} t \otimes \mathrm{d} \mathbb{P} ; H^{2}\left(\mathbb{T}_{L}\right)\right),
$$

where $\hat{w}^{\varepsilon}$ denotes the $\mathrm{d} t \otimes \mathrm{d} \mathbb{P}$-equivalence class of $w^{\varepsilon}$, and

$$
w^{\varepsilon}(t, \cdot)=w_{0}+\int_{0}^{t} A^{\varepsilon} \bar{w}^{\varepsilon}\left(t^{\prime}, \cdot\right) \mathrm{d} t^{\prime}+\int_{0}^{t}\left(B \bar{w}^{\varepsilon}\left(t^{\prime}, \cdot\right)\right) \mathrm{d} W_{H^{2}\left(\mathbb{T}_{L}\right)}\left(t^{\prime}, \cdot\right) \quad \text { for } \quad t \in[0, \delta),
$$

$\mathbb{P}$-almost surely. Here, $\bar{w}^{\varepsilon}$ denotes any $H^{2}\left(\mathbb{T}_{L}\right)$-valued progressively measurable (i.e., for any $t \in[0, \delta)$ the process $\left.\bar{w}^{\varepsilon}\right|_{[0, t] \times \Omega \times \mathbb{T}_{L}}$ is $\mathcal{B}([0, t]) \otimes \mathcal{F}_{t} \otimes \mathcal{B}\left(\mathbb{T}_{L}\right)$-measurable $) \mathrm{d} t \otimes \mathrm{d} \mathbb{P}$-version of $\hat{w}^{\varepsilon}$.

Proposition A.2. Assume that (1.10) holds true and that $p \in[2, \infty)$. Then, for any $w_{0} \in$ $L^{p}\left(\Omega, \mathcal{F}_{0}, \mathbb{P} ; H^{1}\left(\mathbb{T}_{L}\right)\right)$, equation (A.1) has a unique variational solution $w^{\varepsilon}$ with initial value $w_{0}$ satisfying

$$
\mathbb{E}\left(\sup _{t \in[0, \delta)}\left\|w^{\varepsilon}(t, \cdot)\right\|_{1,2}^{p}+\int_{0}^{\delta}\left\|w^{\varepsilon}(t, \cdot)\right\|_{2,2}^{2} \mathrm{~d} t\right)<\infty
$$


Furthermore, we have the a-priori estimates

$$
\begin{aligned}
& \mathbb{E} \sup _{t \in[0, \delta)}\left\|w^{\varepsilon}(t, \cdot)\right\|_{1,2}^{p} \leq C_{1} \mathbb{E}\left\|w_{0}\right\|_{1,2}^{p}, \\
& \lim _{t \nearrow \delta} \mathbb{E}\left\|\partial_{x} w^{\varepsilon}(t, \cdot)\right\|_{2}^{p} \leq e^{C_{2} \delta}\left(\mathbb{E}\left\|\partial_{x} w_{0}\right\|_{2}^{p}+C_{3} \delta \mathbb{E}\left|\int_{0}^{L} w_{0} \mathrm{~d} x\right|^{p}\right),
\end{aligned}
$$

where $C_{1}, C_{2}, C_{3}<\infty$ are independent of $\varepsilon, \delta, w^{\varepsilon}$, and $w_{0}$.

A main ingredient for proving Proposition A.2 is the following lemma, for which the use of Stratonovich calculus (see the discussion in $\S 1.2$ ) is essential:

Lemma A.3 (monotonicity and coercivity). Suppose (1.10) holds true. Then, for $w \in H^{2}\left(\mathbb{T}_{L}\right)$ we have

$$
2\left\langle A^{\varepsilon} w, w\right\rangle+\|B w\|_{L_{2}\left(H^{2}\left(\mathbb{T}_{L}\right) ; L^{2}\left(\mathbb{T}_{L}\right)\right)}^{2} \leq C\|w\|_{2}^{2}-2 \varepsilon\|w\|_{1,2}^{2}
$$

and

$$
2\left\langle\partial_{x} A^{\varepsilon} w, \partial_{x} w\right\rangle+\|B w\|_{L_{2}\left(H^{2}\left(\mathbb{T}_{L}\right) ; \dot{H}^{1}\left(\mathbb{T}_{L}\right)\right)}^{2} \leq C\|w\|_{1,2}^{2}-2 \varepsilon\left\|\partial_{x} w\right\|_{1,2}^{2}
$$

for some $C<\infty$ independent of $w$ and $\varepsilon$, so that in particular

$$
2\left\langle\left\langle A^{\varepsilon} w, w\right\rangle+\|B w\|_{L_{2}\left(H^{2}\left(\mathbb{T}_{L}\right) ; H^{1}\left(\mathbb{T}_{L}\right)\right)}^{2} \leq C\|w\|_{1,2}^{2}-2 \varepsilon\|w\|_{2,2}^{2} .\right.
$$

Proof of Lemma A.3. By definition, estimate (A.9c) follows by adding (A.9a) and (A.9b). We prove (A.9a) and (A.9b) separately:

Proof of (A.9a). Observe that for $w \in H^{2}\left(\mathbb{T}_{L}\right)$ we obtain through integration by parts

$$
\begin{aligned}
\left\langle A^{\varepsilon} w, w\right\rangle & =-\frac{1}{2} \sum_{k \in \mathbb{Z}} \lambda_{k}^{2} \int_{0}^{L} \psi_{k}^{2}\left(\partial_{x} w\right)^{2} \mathrm{~d} x-\varepsilon \int_{0}^{L}\left(\partial_{x} w\right)^{2} \mathrm{~d} x-\frac{1}{8} \sum_{k \in \mathbb{Z}} \lambda_{k}^{2} \int_{0}^{L}\left(\partial_{x} \psi_{k}^{2}\right)\left(\partial_{x} w^{2}\right) \mathrm{d} x \\
& =-\frac{1}{2} \sum_{k \in \mathbb{Z}} \lambda_{k}^{2} \int_{0}^{L} \psi_{k}^{2}\left(\partial_{x} w\right)^{2} \mathrm{~d} x+\frac{1}{8} \sum_{k \in \mathbb{Z}} \lambda_{k}^{2} \int_{0}^{L}\left(\partial_{x}^{2} \psi_{k}^{2}\right) w^{2} \mathrm{~d} x-\varepsilon \int_{0}^{L}\left(\partial_{x} w\right)^{2} \mathrm{~d} x
\end{aligned}
$$

and further

$$
\begin{aligned}
\|B w\|_{L_{2}\left(H^{2}\left(\mathbb{T}_{L}\right) ; L^{2}\left(\mathbb{T}_{L}\right)\right)}^{2} & =\sum_{k \in \mathbb{Z}} \lambda_{k}^{2} \int_{0}^{L}\left(\left(\partial_{x} \psi_{k}\right) w+\psi_{k}\left(\partial_{x} w\right)\right)^{2} \mathrm{~d} x \\
& =\sum_{k \in \mathbb{Z}} \lambda_{k}^{2} \int_{0}^{L} \psi_{k}^{2}\left(\partial_{x} w\right)^{2} \mathrm{~d} x-\sum_{k \in \mathbb{Z}} \lambda_{k}^{2} \int_{0}^{L} \psi_{k}\left(\partial_{x}^{2} \psi_{k}\right) w^{2} \mathrm{~d} x,
\end{aligned}
$$

so that the term $\sum_{k \in \mathbb{Z}} \lambda_{k}^{2} \int_{0}^{L} \psi_{k}^{2}\left(\partial_{x} w\right)^{2} \mathrm{~d} x$ cancels and we get

$$
\begin{aligned}
& 2\left\langle A^{\varepsilon} w, w\right\rangle+\|B w\|_{L_{2}\left(H^{2}\left(\mathbb{T}_{L}\right) ; L^{2}\left(\mathbb{T}_{L}\right)\right)}^{2} \\
& \quad=\frac{1}{4} \sum_{k \in \mathbb{Z}} \lambda_{k}^{2} \int_{0}^{L}\left(\left(\partial_{x}^{2} \psi_{k}^{2}\right)-4 \psi_{k}\left(\partial_{x}^{2} \psi_{k}\right)\right) w^{2} \mathrm{~d} x-2 \varepsilon \int_{0}^{L}\left(\partial_{x} w\right)^{2} \mathrm{~d} x \\
& \stackrel{(1.8),(1.9)}{\leq} C \sum_{k \in \mathbb{Z}} \lambda_{k}^{2}\|w\|_{2}^{2}-2 \varepsilon\left\|\partial_{x} w\right\|_{2}^{2} \stackrel{(1.10)}{\leq} C\|w\|_{2}^{2}-2 \varepsilon\|w\|_{1,2}^{2}
\end{aligned}
$$

for some $C<\infty$ independent of $\varepsilon$, where we have used $\varepsilon \leq 1$. 
Proof of (A.9b). Again, for $w \in H^{2}\left(\mathbb{T}_{L}\right)$ we integrate by parts several times and arrive at

$$
\begin{aligned}
\left\langle\partial_{x} A^{\varepsilon} w, \partial_{x} w\right\rangle \\
=\quad-\frac{1}{2} \sum_{k \in \mathbb{Z}} \lambda_{k}^{2} \int_{0}^{L}\left(\partial_{x}\left(\psi_{k} \partial_{x}\left(\psi_{k} w\right)\right)\right)\left(\partial_{x}^{2} w\right) \mathrm{d} x-\varepsilon \int_{0}^{L}\left(\partial_{x}^{2} w\right)^{2} \mathrm{~d} x \\
=\quad-\frac{1}{2} \sum_{k \in \mathbb{Z}} \lambda_{k}^{2} \int_{0}^{L}\left(\psi_{k}^{2}\left(\partial_{x}^{2} w\right)+\frac{3}{2}\left(\partial_{x} \psi_{k}^{2}\right)\left(\partial_{x} w\right)+\frac{1}{2}\left(\partial_{x}^{2} \psi_{k}^{2}\right) w\right)\left(\partial_{x}^{2} w\right) \mathrm{d} x-\varepsilon \int_{0}^{L}\left(\partial_{x}^{2} w\right)^{2} \mathrm{~d} x \\
=\quad-\frac{1}{2} \sum_{k \in \mathbb{Z}} \lambda_{k}^{2} \int_{0}^{L} \psi_{k}^{2}\left(\partial_{x}^{2} w\right)^{2} \mathrm{~d} x-\frac{3}{8} \sum_{k \in \mathbb{Z}} \lambda_{k}^{2}\left(\partial_{x} \psi_{k}^{2}\right)\left(\partial_{x}\left(\partial_{x} w\right)^{2}\right) \mathrm{d} x \\
\quad+\frac{1}{4} \sum_{k \in \mathbb{Z}} \lambda_{k}^{2} \int_{0}^{L}\left(\partial_{x}^{2} \psi_{k}^{2}\right)\left(\partial_{x} w\right)^{2} \mathrm{~d} x+\frac{1}{8} \sum_{k \in \mathbb{Z}} \lambda_{k}^{2} \int_{0}^{L}\left(\partial_{x}^{3} \psi_{k}^{2}\right)\left(\partial_{x} w^{2}\right) \mathrm{d} x-\varepsilon \int_{0}^{L}\left(\partial_{x}^{2} w\right)^{2} \mathrm{~d} x \\
=-\frac{1}{2} \sum_{k \in \mathbb{Z}} \lambda_{k}^{2} \int_{0}^{L} \psi_{k}^{2}\left(\partial_{x}^{2} w\right)^{2} \mathrm{~d} x+\frac{5}{8} \sum_{k \in \mathbb{Z}} \lambda_{k}^{2} \int_{0}^{L}\left(\partial_{x}^{2} \psi_{k}^{2}\right)\left(\partial_{x} w\right)^{2} \mathrm{~d} x \\
\quad-\frac{1}{8} \sum_{k \in \mathbb{Z}} \lambda_{k}^{2} \int_{0}^{L}\left(\partial_{x}^{4} \psi_{k}^{2}\right) w^{2} \mathrm{~d} x-\varepsilon \int_{0}^{L}\left(\partial_{x}^{2} w\right)^{2} \mathrm{~d} x
\end{aligned}
$$

and

$$
\begin{aligned}
\|B w\|_{L_{2}}^{2}\left(H^{2}\left(\mathbb{T}_{L}\right) ; \dot{H}^{1}\left(\mathbb{T}_{L}\right)\right) & \\
= & \sum_{k \in \mathbb{Z}} \lambda_{k}^{2} \int_{0}^{L}\left(\left(\partial_{x}^{2} \psi_{k}\right) w+2\left(\partial_{x} \psi_{k}\right)\left(\partial_{x} w\right)+\psi_{k}\left(\partial_{x}^{2} w\right)\right)^{2} \mathrm{~d} x \\
= & \sum_{k \in \mathbb{Z}} \lambda_{k}^{2} \int_{0}^{L} \psi_{k}^{2}\left(\partial_{x}^{2} w\right)^{2} \mathrm{~d} x+4 \sum_{k \in \mathbb{Z}} \lambda_{k}^{2} \int_{0}^{L}\left(\partial_{x} \psi_{k}\right)^{2}\left(\partial_{x} w\right)^{2} \mathrm{~d} x+\sum_{k \in \mathbb{Z}} \lambda_{k}^{2} \int_{0}^{L}\left(\partial_{x}^{2} \psi_{k}\right)^{2} w^{2} \mathrm{~d} x \\
& +\sum_{k \in \mathbb{Z}} \lambda_{k}^{2} \int_{0}^{L}\left(\partial_{x} \psi_{k}^{2}\right)\left(\partial_{x}\left(\partial_{x} w\right)^{2}\right) \mathrm{d} x+\sum_{k \in \mathbb{Z}} \lambda_{k}^{2} \int_{0}^{L}\left(\partial_{x}\left(\partial_{x} \psi_{k}\right)^{2}\right)\left(\partial_{x} w^{2}\right) \mathrm{d} x \\
& +2 \sum_{k \in \mathbb{Z}} \lambda_{k}^{2} \int_{0}^{L} \psi_{k}\left(\partial_{x}^{2} \psi_{k}\right) w\left(\partial_{x}^{2} w\right) \mathrm{d} x \\
= & \sum_{k \in \mathbb{Z}} \lambda_{k}^{2} \int_{0}^{L} \psi_{k}^{2}\left(\partial_{x}^{2} w\right)^{2} \mathrm{~d} x+\sum_{k \in \mathbb{Z}} \lambda_{k}^{2} \int_{0}^{L}\left(4\left(\partial_{x} \psi_{k}\right)^{2}-\left(\partial_{x}^{2} \psi_{k}^{2}\right)-2 \psi_{k}\left(\partial_{x}^{2} \psi_{k}\right)\right)\left(\partial_{x} w\right)^{2} \mathrm{~d} x \\
& +\sum_{k \in \mathbb{Z}} \lambda_{k}^{2} \int_{0}^{L}\left(\left(\partial_{x}^{2} \psi_{k}\right)^{2}-\partial_{x}^{2}\left(\partial_{x} \psi_{k}\right)^{2}+\partial_{x}^{2}\left(\psi_{k}\left(\partial_{x}^{2} \psi_{k}\right)\right)\right) w^{2} \mathrm{~d} x \\
= & \sum_{k \in \mathbb{Z}} \lambda_{k}^{2} \int_{0}^{L} \psi_{k}^{2}\left(\partial_{x}^{2} w\right)^{2} \mathrm{~d} x+\sum_{k \in \mathbb{Z}} \lambda_{k}^{2} \int_{0}^{L}\left(2\left(\partial_{x} \psi_{k}\right)^{2}-4 \psi_{k}\left(\partial_{x}^{2} \psi_{k}\right)\right)\left(\partial_{x} w\right)^{2} \mathrm{~d} x \\
& +\sum_{k \in \mathbb{Z}} \lambda_{k}^{2} \int_{0}^{L} \psi_{k}\left(\partial_{x}^{4} \psi_{k}\right) w^{2} \mathrm{~d} x,
\end{aligned}
$$


and hence $\sum_{k \in \mathbb{Z}} \lambda_{k}^{2} \int_{0}^{L} \psi_{k}^{2}\left(\partial_{x}^{2} w\right)^{2} \mathrm{~d} x$ cancels and we arrive at

$$
\begin{aligned}
& 2\left\langle\partial_{x} A^{\varepsilon} w, \partial_{x} w\right\rangle+\|B w\|_{L_{2}\left(H^{2}\left(\mathbb{T}_{L}\right) ; \dot{H}^{1}\left(\mathbb{T}_{L}\right)\right)}^{2} \\
&= \frac{3}{2} \sum_{k \in \mathbb{Z}} \lambda_{k}^{2} \int_{0}^{L}\left(3\left(\partial_{x} \psi_{k}\right)^{2}-\psi_{k}\left(\partial_{x}^{2} \psi_{k}\right)\right)\left(\partial_{x} w\right)^{2} \mathrm{~d} x \\
&+\frac{1}{4} \sum_{k \in \mathbb{Z}} \lambda_{k}^{2} \int_{0}^{L}\left(-\left(\partial_{x}^{4} \psi_{k}^{2}\right)+4 \psi_{k}\left(\partial_{x}^{4} \psi_{k}\right)\right) w^{2} \mathrm{~d} x-2 \varepsilon \int_{0}^{L}\left(\partial_{x}^{2} w\right)^{2} \mathrm{~d} x \\
& \stackrel{(1.8),(1.9)}{\leq} C \sum_{k \in \mathbb{Z}} \lambda_{k}^{2}\|w\|_{1,2}^{2}-2 \varepsilon\left\|\partial_{x}^{2} w\right\|_{2}^{2} \stackrel{(1.10)}{\leq} C\|w\|_{1,2}^{2}-2 \varepsilon\left\|\partial_{x} w\right\|_{1,2}^{2}
\end{aligned}
$$

for some $C<\infty$ independent of $\varepsilon$, where we have used $\varepsilon \leq 1$.

Proof of Proposition A.2. We verify sufficient conditions for variational solutions to (A.1) as can be found for instance in [49, Theorem 4.2.4].

Hemicontinuity. For $u, v, w \in H^{2}\left(\mathbb{T}_{L}\right)$ and $s \in \mathbb{R}$ we have

$$
\left\langle\left\langle A^{\varepsilon}(u+s v), w\right\rangle=\left\langle\left\langle A^{\varepsilon} u, w\right\rangle\right\rangle+s\left\langle\left\langle A^{\varepsilon} v, w\right\rangle,\right.\right.
$$

which is for fixed $u, v$, and $w$ a linear function in $s$ and in particular hemicontinuous.

Weak monotonicity and coercivity. This follows from (A.9c) of Lemma A.3.

Boundedness. For $w \in H^{1}\left(\mathbb{T}_{L}\right)$ and $\varphi \in C^{\infty}\left(\mathbb{T}_{L}\right)$ we have

$$
\begin{aligned}
\mid\left\langle\left\langle A^{\varepsilon} w, \varphi\right\rangle\right| \leq & \frac{1}{2} \sum_{k \in \mathbb{Z}} \lambda_{k}^{2}\left|\int_{0}^{L} \psi_{k}\left(\partial_{x}\left(\psi_{k} w\right)\right)\left(\partial_{x} \varphi\right) \mathrm{d} x\right|+\varepsilon\left|\int_{0}^{L}\left(\partial_{x} w\right)\left(\partial_{x} \varphi\right) \mathrm{d} x\right| \\
& +\frac{1}{2} \sum_{k \in \mathbb{Z}} \lambda_{k}^{2} \mid \int_{0}^{L}\left(\partial_{x}\left(\psi_{k}\left(\partial_{x}\left(\psi_{k} w\right)\right)\right)\left(\partial_{x}^{2} \varphi\right) \mathrm{d} x|+\varepsilon| \int_{0}^{L}\left(\partial_{x}^{2} w\right)\left(\partial_{x}^{2} \varphi\right) \mathrm{d} x \mid\right. \\
\stackrel{(1.8),(1.9)}{\leq} & C\left(\sum_{k \in \mathbb{Z}} \lambda_{k}^{2}+\varepsilon\right)\|w\|_{2,2}\|\varphi\|_{2,2},
\end{aligned}
$$

so that $\left\|A^{\varepsilon} w\right\|_{L^{2}\left(\mathbb{T}_{L}\right)} \stackrel{(1.10)}{\leq} C\|w\|_{2,2}$ since $\varepsilon \leq 1$.

A-priori estimate (A.8a). From [49, Theorem 4.2.4] we infer that a unique variational solution to (A.4) as in Definition A.1 exists and (A.7) is satisfied. While general $p \in[2, \infty)$ are treated in [49, Theorem 5.1.3] or [48, Theorem 1.1], the noise there does not allow for a gradient structure as in the present case. Nonetheless, the reasoning mainly follows the proof of [49, Lemma 5.1.5].

Using Itô's lemma (cf. [45, Theorem 3.1] or [49, Theorem 4.2.5]) and equation (A.6) of Definition A.1, we obtain for $t \in[0, \delta)$

$$
\begin{aligned}
& \left\|w^{\varepsilon}(t, \cdot)\right\|_{1,2}^{2}-\left\|w_{0}\right\|_{1,2}^{2} \\
& =2 \int_{0}^{t}\left(\left(B w^{\varepsilon}\left(t^{\prime}, \cdot\right)\right) \mathrm{d} W_{H^{2}\left(\mathbb{T}_{L}\right)}\left(t^{\prime}\right), w^{\varepsilon}\left(t^{\prime}, \cdot\right)\right)_{1,2} \\
& +\int_{0}^{t}\left(2\left\langle\left\langle A^{\varepsilon} w^{\varepsilon}\left(t^{\prime}, \cdot\right), w^{\varepsilon}\left(t^{\prime}, \cdot\right)\right\rangle\right\rangle+\left\|B w^{\varepsilon}\left(t^{\prime}, \cdot\right)\right\|_{L_{2}\left(H^{2}\left(\mathbb{T}_{L}\right) ; H^{1}\left(\mathbb{T}_{L}\right)\right)}^{2}\right) \mathrm{d} t^{\prime} \\
& \stackrel{\text { (A.3),(A.5) }}{=} 2 \sum_{k \in \mathbb{Z}} \lambda_{k} \int_{0}^{t}\left(\partial_{x}\left(\psi_{k} w^{\varepsilon}\left(t^{\prime}, \cdot\right)\right), w^{\varepsilon}\left(t^{\prime}, \cdot\right)\right)_{1,2} \mathrm{~d} \beta^{k}\left(t^{\prime}\right) \\
& +\int_{0}^{t}\left(2\left\langle\left\langle A^{\varepsilon} w^{\varepsilon}\left(t^{\prime}, \cdot\right), w^{\varepsilon}\left(t^{\prime}, \cdot\right)\right\rangle\right\rangle+\left\|B w^{\varepsilon}\left(t^{\prime}, \cdot\right)\right\|_{L_{2}\left(H^{2}\left(\mathbb{T}_{L}\right) ; H^{1}\left(\mathbb{T}_{L}\right)\right)}^{2}\right) \mathrm{d} t^{\prime},
\end{aligned}
$$


$\mathbb{P}$-almost surely. For $p \geq 4$ this implies again using Itô's lemma for $\mathbb{R} \ni y \mapsto|y|^{\frac{p}{2}}$

(A.10)

$$
\begin{aligned}
& \left\|w^{\varepsilon}(t, \cdot)\right\|_{1,2}^{p}-\left\|w_{0}\right\|_{1,2}^{p} \\
& =\quad p \sum_{k \in \mathbb{Z}} \lambda_{k} \int_{0}^{t}\left\|w^{\varepsilon}\left(t^{\prime}, \cdot\right)\right\|_{1,2}^{p-2}\left(\partial_{x}\left(\psi_{k} w^{\varepsilon}\left(t^{\prime}, \cdot\right)\right), w^{\varepsilon}\left(t^{\prime}, \cdot\right)\right)_{1,2} \mathrm{~d} \beta^{k}\left(t^{\prime}\right) \\
& \quad+\frac{p}{2} \int_{0}^{t}\left\|w^{\varepsilon}\left(t^{\prime}, \cdot\right)\right\|_{1,2}^{p-2}\left(2\left\langle\left\langle A^{\varepsilon} w^{\varepsilon}\left(t^{\prime}, \cdot\right), w^{\varepsilon}\left(t^{\prime}, \cdot\right)\right\rangle\right\rangle+\left\|B w^{\varepsilon}\left(t^{\prime}, \cdot\right)\right\|_{L_{2}\left(H^{2}\left(\mathbb{T}_{L}\right) ; H^{1}\left(\mathbb{T}_{L}\right)\right)}^{2}\right) \mathrm{d} t^{\prime} \\
& \quad+\frac{p(p-2)}{2} \sum_{k \in \mathbb{Z}} \lambda_{k}^{2} \int_{0}^{t}\left\|w^{\varepsilon}\left(t^{\prime}, \cdot\right)\right\|_{1,2}^{p-4}\left(\partial_{x}\left(\psi_{k} w^{\varepsilon}\left(t^{\prime}, \cdot\right)\right), w^{\varepsilon}\left(t^{\prime}, \cdot\right)\right)_{1,2}^{2} \mathrm{~d} t^{\prime},
\end{aligned}
$$

$\mathbb{P}$-almost surely. Next, we introduce for any $R>0$ the stopping times

$$
\tau_{R}:=\inf \left\{t \in[0, \delta):\left\|w^{\varepsilon}(t, \cdot)\right\|_{1,2}>R\right\} \wedge \delta .
$$

By Markov's inequality and using (A.7) for $p=2$

$$
\mathbb{P}\left\{\sup _{t \in[0, \delta)}\left\|w^{\varepsilon}(t, \cdot)\right\|_{1,2}>R\right\} \leq \frac{1}{R^{2}} \mathbb{E} \sup _{t \in[0, \delta)}\left\|w^{\varepsilon}(t, \cdot)\right\|_{1,2}^{2} \rightarrow 0 \quad \text { as } \quad R \rightarrow \infty,
$$

so that $\lim _{R \rightarrow \infty} \tau_{R}=\delta, \mathbb{P}$-almost surely. The Burkholder-Davis-Gundy inequality (cf. [54, Theorem 3.28]) implies

$$
\begin{aligned}
& \mathbb{E} \sup _{t^{\prime} \in\left[0, \tau_{R} \wedge t\right)}\left|\int_{0}^{t^{\prime}}\left\|w^{\varepsilon}\left(t^{\prime \prime}, \cdot\right)\right\|_{1,2}^{p-2}\left(\partial_{x}\left(\psi_{k} w^{\varepsilon}\left(t^{\prime \prime}, \cdot\right)\right), w^{\varepsilon}\left(t^{\prime \prime}, \cdot\right)\right)_{1,2} \mathrm{~d} \beta^{k}\left(t^{\prime \prime}\right)\right| \\
& \leq 3 \mathbb{E} \sqrt{\int_{0}^{\tau_{R} \wedge t}\left\|w^{\varepsilon}\left(t^{\prime}, \cdot\right)\right\|_{1,2}^{2 p-4}\left(\partial_{x}\left(\psi_{k} w^{\varepsilon}\left(t^{\prime}, \cdot\right)\right), w^{\varepsilon}\left(t^{\prime}, \cdot\right)\right)_{1,2}^{2} \mathrm{~d} t^{\prime} .}
\end{aligned}
$$

Now, we note that integration by parts gives

$$
\begin{aligned}
\left(\partial_{x}\left(\psi_{k} w^{\varepsilon}\left(t^{\prime}, \cdot\right)\right), w^{\varepsilon}\left(t^{\prime}, \cdot\right)\right)_{1,2}= & \int_{0}^{L}\left(\partial_{x} \psi_{k}\right)\left(w^{\varepsilon}\right)^{2} \mathrm{~d} x+\int_{0}^{L} \psi_{k} w^{\varepsilon}\left(\partial_{x} w^{\varepsilon}\right) \mathrm{d} x \\
& +\int_{0}^{L}\left(\partial_{x}^{2} \psi_{k}\right) w^{\varepsilon}\left(\partial_{x} w^{\varepsilon}\right) \mathrm{d} x+\frac{3}{2} \int_{0}^{L}\left(\partial_{x} \psi_{k}\right)\left(\partial_{x} w^{\varepsilon}\right)^{2} \mathrm{~d} x,
\end{aligned}
$$

so that with (1.8) and (1.9) we have

$$
\left|\left(\partial_{x}\left(\psi_{k} w^{\varepsilon}\left(t^{\prime}, \cdot\right)\right), w^{\varepsilon}\left(t^{\prime}, \cdot\right)\right)_{1,2}\right| \leq C\left\|w^{\varepsilon}\left(t^{\prime}, \cdot\right)\right\|_{1,2}^{2}, \quad \mathbb{P} \text {-almost surely, }
$$

where $C<\infty$ only depends on $L$, and hence by Young's inequality

$$
\begin{aligned}
& \mathbb{E} \sup _{t^{\prime} \in\left[0, \tau_{R} \wedge t\right)}\left|\int_{0}^{t^{\prime}}\left\|w^{\varepsilon}\left(t^{\prime \prime}, \cdot\right)\right\|_{1,2}^{p-2}\left(\partial_{x}\left(\psi_{k} w^{\varepsilon}\left(t^{\prime \prime}, \cdot\right)\right), w^{\varepsilon}\left(t^{\prime \prime}, \cdot\right)\right)_{1,2} \mathrm{~d} \beta^{k}\left(t^{\prime \prime}\right)\right| \\
& \leq C \mathbb{E} \sqrt{\int_{0}^{\tau_{R} \wedge t}\left\|w^{\varepsilon}\left(t^{\prime}, \cdot\right)\right\|_{1,2}^{2 p} \mathrm{~d} t^{\prime}} \leq C \mathbb{E} \sqrt{\sup _{t^{\prime} \in\left[0, \tau_{R} \wedge t\right)}\left\|w^{\varepsilon}\left(t^{\prime}, \cdot\right)\right\|_{1,2}^{p} \int_{0}^{\tau_{R} \wedge t}\left\|w^{\varepsilon}\left(t^{\prime}, \cdot\right)\right\|_{1,2}^{p} \mathrm{~d} t^{\prime}} \\
& \leq \nu \mathbb{E} \sup _{t^{\prime} \in\left[0, \tau_{R}\right)}\left\|w^{\varepsilon}\left(t^{\prime}, \cdot\right)\right\|_{1,2}^{p}+\frac{C}{\nu} \mathbb{E} \int_{0}^{\tau_{R}}\left\|w^{\varepsilon}\left(t^{\prime}, \cdot\right)\right\|_{1,2}^{p} \mathrm{~d} t^{\prime},
\end{aligned}
$$

where $\nu>0$ can be chosen arbitrarily small and $C<\infty$ is independent of $R$. Furthermore, with the same computation also

$$
\int_{0}^{t}\left\|w^{\varepsilon}\left(t^{\prime}, \cdot\right)\right\|_{1,2}^{p-4}\left(\partial_{x}\left(\psi_{k} w^{\varepsilon}\left(t^{\prime}, \cdot\right)\right), w^{\varepsilon}\left(t^{\prime}, \cdot\right)\right)_{1,2}^{2} \mathrm{~d} t^{\prime} \leq C \int_{0}^{t}\left\|w^{\varepsilon}\left(t^{\prime}, \cdot\right)\right\|_{1,2}^{p} \mathrm{~d} t^{\prime}, \quad \mathbb{P} \text {-almost surely }
$$


where $C<\infty$ only depends on $L$. Now, the combination with (1.10), (A.9c) of Lemma A.3, and (A.10) gives for sufficiently small $\nu$

$$
\mathbb{E} \sup _{t^{\prime} \in\left[0, \tau_{R} \wedge t\right)}\left\|w^{\varepsilon}\left(t^{\prime}, \cdot\right)\right\|_{1,2}^{p} \leq C\left(\mathbb{E}\left\|w_{0}\right\|_{1,2}^{p}+\int_{0}^{\tau_{R} \wedge t} \mathbb{E} \sup _{t^{\prime \prime} \in\left[0, \tau_{R} \wedge t^{\prime}\right)}\left\|w^{\varepsilon}\left(t^{\prime \prime}, \cdot\right)\right\|_{1,2}^{p} \mathrm{~d} t^{\prime \prime}\right),
$$

where $C<\infty$ only depends on $L$ and $p \in\{2\} \cup[4, \infty)$. Grönwall's inequality implies

$$
\mathbb{E} \sup _{t \in\left[0, \tau_{R}\right)}\left\|w^{\varepsilon}(t, \cdot)\right\|_{1,2}^{p} \leq C_{1} \mathbb{E}\left\|w_{0}\right\|_{1,2}^{p},
$$

with $C_{1}<\infty$ only depending on $L$ and $T$, so that (A.8a) for $p \in\{2\} \cup[4, \infty$ ) follows by monotone convergence in the limit as $R \rightarrow \infty$. The case $p \in(2,4)$ is obtained by complex interpolation using the Banach-valued Riesz-Thorin theorem (cf. [37, Theorem 2.2.1] or more generally [5, Theorem 5.1.2]).

A-priori estimate (A.8b). We precisely keep track on the constants appearing in order to derive estimate (A.8b):

With help of Itô's lemma (cf. [45, Theorem 3.1] or [49, Theorem 4.2.5]) we obtain for $t \in[0, \delta$ ) and utilizing equation (A.6) of Definition A.1

$$
\begin{aligned}
& \left\|\partial_{x} w^{\varepsilon}(t, \cdot)\right\|_{2}^{2}-\left\|\partial_{x} w_{0}\right\|_{2}^{2} \\
& \stackrel{(\mathrm{A} .3)}{=} 2 \sum_{k \in \mathbb{Z}} \lambda_{k} \int_{0}^{t}\left(\partial_{x}^{2}\left(\psi_{k} w^{\varepsilon}\left(t^{\prime}, \cdot\right)\right), \partial_{x} w^{\varepsilon}\left(t^{\prime}, \cdot\right)\right)_{2} \mathrm{~d} \beta^{k}\left(t^{\prime}\right) \\
& \quad+\int_{0}^{t}\left(2\left\langle\partial_{x} A^{\varepsilon} w^{\varepsilon}\left(t^{\prime}, \cdot\right), \partial_{x} w^{\varepsilon}\left(t^{\prime}, \cdot\right)\right\rangle+\left\|B w^{\varepsilon}\left(t^{\prime}, \cdot\right)\right\|_{L_{2}\left(H^{2}\left(\mathbb{T}_{L}\right) ; \dot{H}^{1}\left(\mathbb{T}_{L}\right)\right)}^{2}\right) \mathrm{d} t^{\prime},
\end{aligned}
$$

$\mathbb{P}$-almost surely. For $p \geq 4$, Itô's formula applied to $\mathbb{R} \ni y \mapsto|y|^{\frac{p}{2}}$ gives

$$
\begin{aligned}
&\left\|\partial_{x} w^{\varepsilon}(t, \cdot)\right\|_{2}^{p}-\left\|\partial_{x} w_{0}\right\|_{2}^{p} \\
&= p \sum_{k \in \mathbb{Z}} \lambda_{k} \int_{0}^{t}\left\|\partial_{x} w^{\varepsilon}\left(t^{\prime}, \cdot\right)\right\|_{2}^{p-2}\left(\partial_{x}^{2}\left(\psi_{k} w^{\varepsilon}\left(t^{\prime}, \cdot\right)\right), \partial_{x} w^{\varepsilon}\left(t^{\prime}, \cdot\right)\right)_{2} \mathrm{~d} \beta^{k}\left(t^{\prime}\right) \\
&+\frac{p}{2} \int_{0}^{t}\left\|\partial_{x} w^{\varepsilon}\left(t^{\prime}, \cdot\right)\right\|_{2}^{p-2}\left(2\left\langle\partial_{x} A^{\varepsilon} w^{\varepsilon}\left(t^{\prime}, \cdot\right), \partial_{x} w^{\varepsilon}\left(t^{\prime}, \cdot\right)\right\rangle+\left\|B w^{\varepsilon}\left(t^{\prime}, \cdot\right)\right\|_{L_{2}\left(H^{2}\left(\mathbb{T}_{L}\right) ; \dot{H}^{1}\left(\mathbb{T}_{L}\right)\right)}^{2}\right) \mathrm{d} t^{\prime} \\
&+\frac{p(p-2)}{2} \sum_{k \in \mathbb{Z}} \lambda_{k}^{2} \int_{0}^{t}\left\|\partial_{x} w^{\varepsilon}\left(t^{\prime}, \cdot\right)\right\|_{2}^{p-4}\left(\partial_{x}^{2}\left(\psi_{k} w^{\varepsilon}\left(t^{\prime}, \cdot\right)\right), \partial_{x} w^{\varepsilon}\left(t^{\prime} \cdot \cdot\right)\right)_{2}^{2} \mathrm{~d} t^{\prime},
\end{aligned}
$$

$\mathbb{P}$-almost surely. Taking the expectation gives

$$
\begin{aligned}
\mathbb{E} \| & \partial_{x} w^{\varepsilon}(t, \cdot)\left\|_{2}^{p}-\mathbb{E}\right\| \partial_{x} w^{\varepsilon}(0, \cdot) \|_{2}^{p} \\
= & \frac{p}{2} \mathbb{E} \int_{0}^{t}\left\|\partial_{x} w^{\varepsilon}\left(t^{\prime}, \cdot\right)\right\|_{2}^{p-2}\left(2\left\langle\partial_{x} A^{\varepsilon} w^{\varepsilon}\left(t^{\prime}, \cdot\right), \partial_{x} w^{\varepsilon}\left(t^{\prime}, \cdot\right)\right\rangle+\left\|B w^{\varepsilon}\left(t^{\prime}, \cdot\right)\right\|_{L_{2}\left(H^{2}\left(\mathbb{T}_{L}\right) ; \dot{H}^{1}\left(\mathbb{T}_{L}\right)\right)}^{2}\right) \mathrm{d} t^{\prime} \\
& +\frac{p(p-2)}{2} \sum_{k \in \mathbb{Z}} \lambda_{k}^{2} \mathbb{E} \int_{0}^{t}\left\|\partial_{x} w^{\varepsilon}\left(t^{\prime}, \cdot\right)\right\|_{2}^{p-4}\left(\partial_{x}^{2}\left(\psi_{k} w^{\varepsilon}\left(t^{\prime}, \cdot\right)\right), \partial_{x} w^{\varepsilon}\left(t^{\prime}, \cdot\right)\right)_{2}^{2} \mathrm{~d} t^{\prime} .
\end{aligned}
$$

For the last line observe that through integration by parts as before

$$
\left(\partial_{x}^{2}\left(\psi_{k} w^{\varepsilon}\left(t^{\prime}, \cdot\right)\right), \partial_{x} w^{\varepsilon}\left(t^{\prime}, \cdot\right)\right)_{2}=\int_{0}^{L}\left(\partial_{x}^{2} \psi_{k}\right) w^{\varepsilon} \partial_{x} w^{\varepsilon} \mathrm{d} x+\frac{3}{2} \int_{0}^{L}\left(\partial_{x} \psi_{k}\right)\left(\partial_{x} w^{\varepsilon}\right)^{2} \mathrm{~d} x,
$$

$\mathbb{P}$-almost surely, that is,

$$
\left(\partial_{x}^{2}\left(\psi_{k} w^{\varepsilon}\left(t^{\prime}, \cdot\right)\right), \partial_{x} w^{\varepsilon}\left(t^{\prime}, \cdot\right)\right)_{2}^{2} \stackrel{(1.8),(1.9)}{\leq} C\left\|\partial_{x} w^{\varepsilon}\left(t^{\prime}, \cdot\right)\right\|_{2}^{2}\left\|w^{\varepsilon}\left(t^{\prime}, \cdot\right)\right\|_{1,2}^{2}, \quad \mathbb{P} \text {-almost surely }
$$


Further applying (A.9b) of Lemma A.3 gives

$$
\begin{aligned}
& \mathbb{E}\left\|\partial_{x} w^{\varepsilon}(t, \cdot)\right\|_{2}^{p}-\mathbb{E}\left\|\partial_{x} w^{\varepsilon}(0, \cdot)\right\|_{2}^{p} \\
& \quad \leq \quad C p(p+1) \sum_{k \in \mathbb{Z}} \lambda_{k}^{2} \mathbb{E} \int_{0}^{t}\left\|\partial_{x} w^{\varepsilon}\left(t^{\prime}, \cdot\right)\right\|_{2}^{p-2}\left(\left\|\partial_{x} w^{\varepsilon}\left(t^{\prime}, \cdot\right)\right\|_{2}^{2}+\left(\int_{0}^{L} w^{\varepsilon}\left(t^{\prime}, \cdot\right) \mathrm{d} x\right)^{2}\right) \mathrm{d} t^{\prime} \\
& \stackrel{(1.10)}{\leq} \quad C_{2} \int_{0}^{t} \mathbb{E}\left\|\partial_{x} w^{\varepsilon}\left(t^{\prime}, \cdot\right)\right\|_{2}^{p} \mathrm{~d} t^{\prime}+C_{3} \mathbb{E} \int_{0}^{t}\left|\int_{0}^{L} w^{\varepsilon}\left(t^{\prime}, \cdot\right) \mathrm{d} x\right|^{p} \mathrm{~d} t^{\prime},
\end{aligned}
$$

where we have applied Poincaré's inequality

$$
\left\|w^{\varepsilon}\left(t^{\prime}, \cdot\right)\right\|_{1,2} \leq C\left(\left\|\partial_{x} w^{\varepsilon}\left(t^{\prime}, \cdot\right)\right\|_{2}+\left|\int_{0}^{L} w^{\varepsilon}\left(t^{\prime}, \cdot\right) \mathrm{d} x\right|\right), \quad \text { P-almost surely, }
$$

and Young's inequality. Testing of (A.6) of Definition A.1 against a non-trivial constant gives $\int_{0}^{L} w^{\varepsilon}(t, \cdot) \mathrm{d} x=\int_{0}^{L} w_{0} \mathrm{~d} x$ for $t \in[0, T), \mathbb{P}$-almost surely. Now the claim (A.8) for $p \in\{2\} \cup[4, \infty)$ follows from Grönwall's inequality and the general case $p \in[2, \infty)$ by complex interpolation using the Banach-valued Riesz-Thorin theorem (cf. [37, Theorem 2.2.1] or more generally [5, Theorem 5.1.2]).

\section{Appendix B. Real interpolation of Besov spaCes With mixed Smoothness}

The following result from interpolation theory is essential in proving regularity in time (cf. Proposition 4.2).

Lemma B.1. Suppose $\delta \in(0, \infty), r_{1}, r_{2}, s_{1}, s_{2} \in \mathbb{R}$ with $r_{1} \neq r_{2}$ and $s_{1} \neq s_{2}, q \in[1, \infty)$, and $\kappa \in[0,1]$. Then

$$
\left(B_{q}^{r_{1}, q}\left([0, \delta) ; B_{q}^{s_{1}, q}\left(\mathbb{T}_{L}\right)\right), B_{q}^{r_{2}, q}\left([0, \delta) ; B_{q}^{s_{2}, q}\left(\mathbb{T}_{L}\right)\right)\right)_{\kappa, q}=B_{q}^{r, q}\left([0, \delta) ; B_{q}^{s, q}\left(\mathbb{T}_{L}\right)\right),
$$

where $r=(1-\kappa) r_{1}+\kappa r_{2}$ and $s=(1-\kappa) s_{1}+\kappa s_{2}$. The norms in (B.1) are equivalent, with bounds that are independent of $\delta$.

Proof. By [1, Proposition 4.2] and scaling in the time variable, there exists a $\delta$-uniformly bounded linear extension operator

$$
E: Y_{j}:=B_{q}^{r_{j}, q}\left([0, \delta) ; B_{q}^{s_{j}, q}\left(\mathbb{T}_{L}\right)\right) \rightarrow Z_{j}:=B_{q}^{r_{j}, q}\left(\mathbb{R} ; B_{q}^{s_{j}, q}\left(\mathbb{T}_{L}\right)\right),
$$

that is, setting $R(\cdot):=\left.(\cdot)\right|_{[0, \delta)}$, we have $R E v=v$ for $v \in Y_{j}$ and the operator norm of $E$ is independent of $\delta$. Now, we may apply [1, Theorem 3.1], [2, Theorem 2.7.2 (i)], or [31, (6.9)] to deduce

$$
\left(Z_{1}, Z_{2}\right)_{\kappa, q}=B_{q}^{r, q}\left(\mathbb{R} ;\left(B_{q}^{s_{1}, q}\left(\mathbb{T}_{L}\right), B_{q}^{s_{2}, q}\left(\mathbb{T}_{L}\right)\right)_{\kappa, q}\right) .
$$

The interpolation of periodic Besov spaces is known (cf. [52, §3.6.1, Theorem 1 (i)]), that is,

$$
\left(B_{q}^{s_{1}, q}\left(\mathbb{T}_{L}\right), B_{q}^{s_{2}, q}\left(\mathbb{T}_{L}\right)\right)_{\kappa, q}=B_{q}^{s, q}\left(\mathbb{T}_{L}\right) .
$$

Altogether,

$$
Y_{j}=R\left(E Y_{j}\right)=R Z_{j}=R B_{q}^{r, q}\left(\mathbb{R} ; B_{q}^{s, q}\left(\mathbb{T}_{L}\right)\right)=B_{q}^{r, q}\left([0, \delta) ; B_{q}^{s, q}\left(\mathbb{T}_{L}\right)\right)
$$

with $\delta$-uniformly equivalent norms, which yields (B.1).

Lemma B.2. Suppose that $X$ is a Banach space, $T \in(0, \infty), N \in \mathbb{N}, \delta:=\frac{T}{N}, s \in(0,1)$, and $q \in(1, \infty]$. If $\phi \in B C^{0}([0, T) ; X)$ and $\phi \in B_{q}^{s, q}\left(\left[(j-1) \frac{\delta}{2}, j \frac{\delta}{2}\right) ; X\right)$ for $j \in\{1, \ldots, 2 N\}$, then $\phi \in B_{q}^{s, q}([0, T) ; X)$ with

$$
\|\phi\|_{B_{q}^{s, q}([0, T) ; X)} \leq C\left(\sum_{j=1}^{2 N}\|\phi\|_{B_{q}^{s, q}\left(\left[(j-1) \frac{\delta}{2}, j \frac{\delta}{2}\right) ; X\right)}^{q}\right)^{\frac{1}{q}},
$$

where $C<\infty$ only depends on $q$. 
Proof. By mollification with a standard mollifier and using the interpolation property, we see that we can approximate $\phi$ on any interval $\left[(j-1) \frac{\delta}{2}, j \frac{\delta}{2}\right)$ by a function $\phi_{\varepsilon} \in C^{\infty}\left(\left[(j-1) \frac{\delta}{2}, j \frac{\delta}{2}\right) ; X\right)$ with

$$
\left\|\phi-\phi_{\varepsilon}\right\|_{B C^{0}\left(\left[(j-1) \frac{\delta}{2}, j \frac{\delta}{2}\right) ; X\right)} \rightarrow 0 \quad \text { and } \quad\left\|\phi-\phi_{\varepsilon}\right\|_{B_{q}^{s, q}\left(\left[(j-1) \frac{\delta}{2}, j \frac{\delta}{2}\right) ; X\right)} \quad \text { as } \quad \varepsilon \searrow 0,
$$

where $j \in\{1, \ldots, 2 N\}$. Adding to $\phi_{\varepsilon}$ the polygonal chain through the points

$$
\left(j \frac{\delta}{2}, \phi\left(j \frac{\delta}{2}, \cdot\right)-\phi_{\varepsilon}\left(j \frac{\delta}{2}, \cdot\right)\right), \quad \text { where } j \in\{0, \ldots, 2 N\},
$$

we may without loss of generality additionally assume $\phi_{\varepsilon} \in B C^{0}([0, T) ; X)$, so that in particular $\phi_{\varepsilon} \in W^{1, \infty}([0, T) ; X)$, and

$$
\left\|\phi-\phi_{\varepsilon}\right\|_{B C^{0}([0, T) ; X)} \rightarrow 0 \text { as } \varepsilon \searrow 0 .
$$

Since up to a $q$-dependent constant $\left(\right.$ denoted by $\left.\sim_{q}\right)$ we have for $\psi \in W^{1, \infty}([0, T) ; X)$,

$$
\|\psi\|_{B_{q}^{s, q}([0, T) ; X)}^{q} \sim_{q} \int_{0}^{\infty} \inf _{\psi=\psi_{1}+\psi_{2}}\left(\tau^{-s q}\left\|\psi_{1}\right\|_{L^{q}([0, T) ; X)}^{q}+\tau^{(1-s) q}\left\|\psi_{2}\right\|_{W^{1, q}([0, T) ; X)}^{q}\right) \frac{\mathrm{d} \tau}{\tau},
$$

we recognize that by Sobolev embedding $\psi_{2} \in B C^{0}([0, T) ; X)$, so that any decomposition $\psi=$ $\psi_{1}+\psi_{2}$ with $\psi_{1} \in L^{q}([0, T) ; X)$ and $\psi_{2} \in W^{1, q}([0, T) ; X)$ induces a decomposition $\psi=\psi_{1}+\psi_{2}$ with $\psi_{1} \in L^{q}\left(\left[(j-1) \frac{\delta}{2}, j \frac{\delta}{2}\right) ; X\right)$ and $\psi_{2} \in W^{1, q}\left(\left[(j-1) \frac{\delta}{2}, j \frac{\delta}{2}\right) ; X\right)$ for all $j \in\{1, \ldots, 2 N\}$. Hence, we can conclude that

$$
\begin{aligned}
& \|\psi\|_{B_{q}^{s, q}([0, T) ; X)}^{q} \\
& \quad \leq C \sum_{j=1}^{2 N} \int_{0}^{\infty} \inf _{\psi=\psi_{1}+\psi_{2}}\left(\tau^{-s q}\left\|\psi_{1}\right\|_{L^{q}\left(\left[(j-1) \frac{\delta}{2}, j \frac{\delta}{2}\right) ; X\right)}^{q}+\tau^{(1-s) q}\left\|\psi_{2}\right\|_{W^{1, q}\left(\left[(j-1) \frac{\delta}{2}, j \frac{\delta}{2}\right) ; X\right)}^{q}\right) \frac{\mathrm{d} \tau}{\tau} \\
& \quad \sim_{q} \sum_{j=1}^{2 N}\|\psi\|_{B_{q}^{s, q}\left(\left[(j-1) \frac{\delta}{2}, j \frac{\delta}{2}\right) ; X\right)}^{q} .
\end{aligned}
$$

This implies $\left\|\phi_{\varepsilon}-\phi_{\varepsilon^{\prime}}\right\|_{B_{q}^{s, q}([0, T) ; X)} \rightarrow 0$ as $\varepsilon, \varepsilon^{\prime} \searrow 0$, so that $\phi \in B_{q}^{s, q}([0, T) ; X)$ and (B.2) holds true.

\section{REFERENCES}

[1] Herbert Amann. Compact embeddings of vector-valued Sobolev and Besov spaces. volume 35(55), pages 161-177. 2000. Dedicated to the memory of Branko Najman.

[2] Herbert Amann. Linear and quasilinear parabolic problems. Vol. II, volume 106 of Monographs in Mathematics. Birkhäuser/Springer, Cham, 2019. Function spaces.

[3] Alain Bensoussan, Roland Glowinski, and Aurel Răşcanu. Approximation of the Zakai equation by the splitting up method. SIAM J. Control Optim., 28(6):1420-1431, 1990.

[4] Elena Beretta, Michiel Bertsch, and Roberta Dal Passo. Nonnegative solutions of a fourth-order nonlinear degenerate parabolic equation. Arch. Rational Mech. Anal., 129(2):175-200, 1995.

[5] Jöran Bergh and Jörgen Löfström. Interpolation spaces. An introduction. Springer-Verlag, Berlin-New York, 1976. Grundlehren der Mathematischen Wissenschaften, No. 223.

[6] Francisco Bernis and Avner Friedman. Higher order nonlinear degenerate parabolic equations. J. Differential Equations, 83(1):179-206, 1990.

[7] Andrew J. Bernoff and Thomas P. Witelski. Linear stability of source-type similarity solutions of the thin film equation. Appl. Math. Lett., 15(5):599-606, 2002.

[8] A. L. Bertozzi and M. Pugh. The lubrication approximation for thin viscous films: regularity and long-time behavior of weak solutions. Comm. Pure Appl. Math., 49(2):85-123, 1996.

[9] Björn Bringmann, Lorenzo Giacomelli, Hans Knüpfer, and Felix Otto. Corrigendum to "Smooth zero-contactangle solutions to a thin-film equation around the steady state" [J. Differential Equations 245 (2008) 14541506]. J. Differential Equations, 261(2):1622-1635, 2016.

[10] Eric A. Carlen and Süleyman Ulusoy. Asymptotic equipartition and long time behavior of solutions of a thin-film equation. J. Differential Equations, 241(2):279-292, 2007.

[11] Eric A. Carlen and Süleyman Ulusoy. Localization, smoothness, and convergence to equilibrium for a thin film equation. Discrete Contin. Dyn. Syst., 34(11):4537-4553, 2014.

[12] José A. Carrillo and Giuseppe Toscani. Long-time asymptotics for strong solutions of the thin film equation. Comm. Math. Phys., 225(3):551-571, 2002. 
[13] Federico Cornalba. A priori positivity of solutions to a non-conservative stochastic thin-film equation. arXiv:1811.07826, page 16, 2018.

[14] Roberta Dal Passo, Harald Garcke, and Günther Grün. On a fourth-order degenerate parabolic equation: global entropy estimates, existence, and qualitative behavior of solutions. SIAM J. Math. Anal., 29(2):321$342,1998$.

[15] Konstantinos Dareiotis and Benjamin Gess. Nonlinear diffusion equations with nonlinear gradient noise. arXiv:1811.08356, page 42, 2018.

[16] Konstantinos Dareiotis and Benjamin Gess. Supremum estimates for degenerate, quaslinear stochastic partial differential equations. to appear in Ann. Henri Poincaré (B) Probab. Statist. (arXiv:1712.06655), pages 1-35, 2018.

[17] Benny Davidovitch, Esteban Moro, and Howard A. Stone. Spreading of viscous fluid drops on a solid substrate assisted by thermal fluctuations. Phys. Rev. Lett., 95:244505, Dec 2005.

[18] Sergey Degtyarev. Classical solvability of the multidimensional free boundary problem for the thin film equation with quadratic mobility in the case of partial wetting. Discrete Contin. Dyn. Syst., 37(7):36253699, 2017.

[19] Julian Fischer and Günther Grün. Existence of positive solutions to stochastic thin-film equations. SIAM J. Math. Anal., 50(1):411-455, 2018.

[20] Franco Flandoli and Dariusz Gątarek. Martingale and stationary solutions for stochastic Navier-Stokes equations. Probab. Theory Related Fields, 102(3):367-391, 1995.

[21] Máté Gerencsér, István Gyöngy, and Nicolai Krylov. On the solvability of degenerate stochastic partial differential equations in Sobolev spaces. Stoch. Partial Differ. Equ. Anal. Comput., 3(1):52-83, 2015.

[22] Benjamin Gess and Benjamin Fehrman. Well-posedness of nonlinear diffusion equations with nonlinear, conservative noise. arXiv:1712.05775, page 57, 2019.

[23] Benjamin Gess and Panagiotis E. Souganidis. Scalar conservation laws with multiple rough fluxes. Commun. Math. Sci., 13(6):1569-1597, 2015.

[24] Lorenzo Giacomelli, Manuel V. Gnann, Hans Knüpfer, and Felix Otto. Well-posedness for the Navier-slip thin-film equation in the case of complete wetting. J. Differential Equations, 257(1):15-81, 2014.

[25] Lorenzo Giacomelli and Hans Knüpfer. A free boundary problem of fourth order: classical solutions in weighted Hölder spaces. Comm. Partial Differential Equations, 35(11):2059-2091, 2010.

[26] Lorenzo Giacomelli, Hans Knüpfer, and Felix Otto. Smooth zero-contact-angle solutions to a thin-film equation around the steady state. J. Differential Equations, 245(6):1454-1506, 2008.

[27] Manuel V. Gnann. Well-posedness and self-similar asymptotics for a thin-film equation. SIAM J. Math. Anal., 47(4):2868-2902, 2015.

[28] Manuel V. Gnann. On the regularity for the Navier-slip thin-film equation in the perfect wetting regime. Arch. Ration. Mech. Anal., 222(3):1285-1337, 2016.

[29] Manuel V. Gnann and Mircea Petrache. The Navier-slip thin-film equation for 3D fluid films: existence and uniqueness. J. Differential Equations, 265(11):5832-5958, 2018.

[30] T. E. Govindan. On Trotter-Kato approximations of semilinear stochastic evolution equations in infinite dimensions. Statist. Probab. Lett., 96:299-306, 2015.

[31] Pierre Grisvard. Commutativité de deux foncteurs d'interpolation et applications. J. Math. Pures Appl. (9), 45:207-290, 1966.

[32] Günther Grün. Droplet spreading under weak slippage - existence for the Cauchy problem. Comm. Partial Differential Equations, 29(11-12):1697-1744, 2004.

[33] Günther Grün, Klaus Mecke, and Markus Rauscher. Thin-film flow influenced by thermal noise. J. Stat. Phys., 122(6):1261-1291, 2006.

[34] István Gyöngy and Nicolai Krylov. On the splitting-up method and stochastic partial differential equations. Ann. Probab., 31(2):564-591, 2003.

[35] Martina Hofmanová. Degenerate parabolic stochastic partial differential equations. Stochastic Process. Appl., 123(12):4294-4336, 2013.

[36] Luca Hornung. Quasilinear Parabolic Stochastic Evolution Equations Via Maximal $L^{p}$-Regularity. Potential Anal., 50(2):279-326, 2019.

[37] Tuomas Hytönen, Jan van Neerven, Mark Veraar, and Lutz Weis. Analysis in Banach spaces. Vol. I. Martingales and Littlewood-Paley theory, volume 63 of Ergebnisse der Mathematik und ihrer Grenzgebiete. 3. Folge. A Series of Modern Surveys in Mathematics [Results in Mathematics and Related Areas. 3rd Series. A Series of Modern Surveys in Mathematics]. Springer, Cham, 2016.

[38] A. Jakubowski. The almost sure Skorokhod representation for subsequences in nonmetric spaces. Teor. Veroyatnost. i Primenen., 42(1):209-216, 1997.

[39] Dominik John. On uniqueness of weak solutions for the thin-film equation. J. Differential Equations, 259(8):4122-4171, 2015.

[40] Achim Klenke. Probability theory. Universitext. Springer-Verlag London, Ltd., London, 2008. A comprehensive course, Translated from the 2006 German original.

[41] Hans Knüpfer. Well-posedness for the Navier slip thin-film equation in the case of partial wetting. Comm. Pure Appl. Math., 64(9):1263-1296, 2011. 
[42] Hans Knüpfer. Well-posedness for a class of thin-film equations with general mobility in the regime of partial wetting. Arch. Ration. Mech. Anal., 218(2):1083-1130, 2015.

[43] Hans Knüpfer. Erratum to: Well-posedness for a class of thin-film equations with general mobility in the regime of partial wetting. in preparation, 2019.

[44] N. V. Krylov and B. L. Rozovskiı̌. Characteristics of second-order degenerate parabolic Itô equations. Trudy Sem. Petrovsk., (8):153-168, 1982.

[45] Nikolai V. Krylov. A relatively short proof of Itô's formula for SPDEs and its applications. Stochastic Partial Differential Equations: Analysis and Computations, 1(1):152-174, 2013.

[46] Nikolai V. Krylov and Boris L. Rozovskii. Stochastic evolution equations. Itogi Nauki i Tekhniki. Seriya" Sovremennye Problemy Matematiki. Noveishie Dostizheniya", 14:71-146, 1979.

[47] J.-L. Lions and J. Peetre. Sur une classe d'espaces d'interpolation. Inst. Hautes Études Sci. Publ. Math., (19):5-68, 1964.

[48] Wei Liu and Michael Röckner. SPDE in Hilbert space with locally monotone coefficients. J. Funct. Anal., 259(11):2902-2922, 2010.

[49] Wei Liu and Michael Röckner. Stochastic partial differential equations: an introduction. Universitext. Springer, Cham, 2015.

[50] Daniel Matthes, Robert J. McCann, and Giuseppe Savaré. A family of nonlinear fourth order equations of gradient flow type. Comm. Partial Differential Equations, 34(10-12):1352-1397, 2009.

[51] Robert J. McCann and Christian Seis. The spectrum of a family of fourth-order nonlinear diffusions near the global attractor. Comm. Partial Differential Equations, 40(2):191-218, 2015.

[52] Hans-Jürgen Schmeisser and Hans Triebel. Topics in Fourier analysis and function spaces. A WileyInterscience Publication. John Wiley \& Sons, Ltd., Chichester, 1987.

[53] Christian Seis. The thin-film equation close to self-similarity. Anal. PDE, 11(5):1303-1342, 2018.

[54] Steven E. Shreve and Ioannis Karatzas. Brownian motion and stochastic calculus. New York, Berlin, Heidelberg, London, Paris, Tokyo, 1991.

[55] Anatolii V. Skorokhod. On the limiting transition from a sequence of sums of independent random quantities to a homogeneous random process with independent increments. Dokl. Akad. Nauk SSSR (N.S.), 104:364$367,1955$.

[56] Hans Triebel. Interpolation theory, function spaces, differential operators, volume 18 of North-Holland Mathematical Library. North-Holland Publishing Co., Amsterdam-New York, 1978.

[57] Jan van Neerven, Mark Veraar, and Lutz Weis. Maximal $L^{p}$-regularity for stochastic evolution equations. SIAM J. Math. Anal., 44(3):1372-1414, 2012.

[58] Jan van Neerven, Mark Veraar, and Lutz Weis. Stochastic maximal $L^{p}$-regularity. Ann. Probab., 40(2):788$812,2012$.

(Benjamin Gess) Max Planck Institute for Mathematics in the Sciences, Inselstr. 22, 04103 Leipzig, Germany and Faculty of Mathematics, Bielefeld University, Universitätsstr. 25, 33615 BieleFELD, GERMANY

E-mail address: b.gess@mis.mpg.de

(Manuel V. Gnann) Delft Institute of Applied Mathematics, Faculty of Electrical Engineering, Mathematics and Computer Science, Delft University of Technology, Van Mourik Broekmanweg 6, 2628 XE Delft, Netherlands

E-mail address: M.V.Gnann@tudelft.nl 